\title{
As Álgebras Plena de Colombeau e de Aragona:
}

\section{Conjuntos Internos e Aplicações}

\author{
Jailson Calado da Silva \\ TESE APRESENTADA \\ AO \\ Instituto DE MATEMÁtica E EstatísticA \\ $\mathrm{DA}$ \\ Universidade DE SÃo PAUlo \\ PARA \\ OBTENÇÃO DO TÍTULO \\ $\mathrm{DE}$ \\ Doutor EM CIÊNCIAS \\ Programa: Matemática \\ Orientador: Prof. Dr. Orlando Stanley Juriaans
}

Durante o desenvolvimento deste trabalho o autor recebeu auxílio financeiro da CAPES

São Paulo, Novembro de 2019 


\title{
As Álgebras Plena de Colombeau e de Aragona:
}

\author{
Conjuntos Internos e Aplicações
}

Esta é a versão original da tese elaborada pelo candidato Jailson Calado da Silva, tal como submetida à Comissão Julgadora. 


\title{
As Álgebras Plena de Colombeau e de Aragona:
}

\author{
Conjuntos Internos e Aplicações
}

Esta versão da tese contém as correções e alterações sugeridas pela Comissão Julgadora durante a defesa da versão original do trabalho, realizada em 27/11/2019. Uma cópia da versão original está disponível no Instituto de Matemática e Estatástica da Universidade de São Paulo.

Comissão Julgadora:

- Prof. Dr. Orlando Stanley Juriaans (orientador)- MAT-IME-USP

- Prof. Dr. Raul Antonio Ferraz - MAT-IME-USP

- Prof. Dr. Manuel Antolino Milla Miranda-UEPB

- Prof. Dr. Francisco Villarreal Alvarado-UNESP

- Prof. Dr. Wagner de Oliveira Cortes-UFRGS 
Ao meu querido irmão Jadison Calado da Silva, que nos deixou há pouco tempo, mas fez muito por mim ao longo da sua vida. 


\section{Agradecimentos}

Quero primeiro agradecer a Deus por permitir a conclusão desse trabalho, pois tive muitos ventos que se moveram contrário aos meus propósitos, então agradeço a Deus pelo fôlego de vida e pela presença do espírito santo na minha vida, pois durante o doutorado tive experiências com este espírito santo.

Faço mensão agora de algumas pessoas que contribuíram direto, ou indiretamente para o meu sucesso. Agradeço minha família na pessoa da minha mãe Edi Calado Silva, minha querida irmã Edna Calado da Silva, e preciso fazer uma ênfase para meu irmão Jadison Calado da Silva, pois foi uma grande pessoa que veio a esse mundo para auxiliar-me em tudo, o mesmo aconselhou a estudar e contribuiu muito para meu progresso, meu irmão falaceu quando eu estava no último ano do doutorado, e aqui deixo toda minha gratidão e dedico a tese em memória à ele. Durante toda minha vida tive muitas batalhas para enfrentar, contudo passaram na minha vida muitas pessoas auxiliando e ajudando de alguma forma. A família Soares me ajudaram muito, na pessoa de José Soares e Francisca Fernandes Soares, eles são meus padrinhos do batismo da igreja católica. Grandes amigos me apoiaram durante os momentos difíceis como Samuel Assunção, Fernando Lopes, Sidione Alves da Luz, Jucelino Sobreiro e não poderia deixar de fazer uma mensão dedicada ao Reginaldo Felix pois o mesmo é um grande amigo.

Uma família que foram muito presente na minha vida participando e auxiliando no doutorado, foi a família que mora no Rio de Janeiro do amigo Paulo Cristiano Queiroz Moraes, quando comecei meu doutorado no impa, essa família foi acolhedora na pessoa do Marcos Ribeiro de Oliveira Neto, Lucinete Galvão de Oliveira sua esposa e Mauricio de Oliveira Queiroz tio do Paulo Cristiano, agradeço também o Henrique filho da Lucinete Galvão, Lenadro e Ninão por todo auxílio.

$\mathrm{Na}$ área acadêmica tiveram grandes personagens que cooperaram, primeiro alunos do mestrado da UFMA, auxiliaram de alguma forma, que são Ronaldo Smith, Paulo Cristiano Queiroz Moraes, Marco Azevedo, José Santana e Marlon Cesar, Jadevilson Cruz. Quando decidi fazer meu doutorado também tive apoio de alguns professores, portanto quero agradecer o auxílio de Nivaldo Muniz por ajudar-me a chegar no impa para participar do semestre temático em sistemas dinâmicos, agradeço o professor Marco Araújo pois aconselhou que fosse fazer doutorado.

Quando fui se inscrever no doutorado tive apoio de dois grandes professores que quero dar destaques, são eles o professor José Antonio Pires Ferreira Marão, e a professora Valeska 
Martins de Souza, pois os mesmos me auxiliaram em grande parte para eu chegar no IMEUSP.

No doutorado em IME-USP foram momentos de muitos crescimentos, e apesar de ter tido vários ventos que sopraram contrário ao meu favor, com algumas amizades de puro enganos, e desacerto com a orientação de orientadores anteriores, considero esses momentos necessários para podermos evoluirmos de alguma forma. Então faço mensão de alguns amigos colaboradores durante o doutorado como Jadevilson Cruz Ribeiro, Eurípedes Carvalho da Silva, João Nunes de Araújo Neto, Carlos Gomes, Kaique Matias de Andrade Roberto, Daniel de Brito Reis, Guilherme Porfírio Marson, Yuri Sampaio Maluf, Arcelino Bruno Lobato do nascimento, Rodrigo Silva dos Santos e Oscar Armando Hernándes Morales, Elivaldo, João Coelho, Francisco, Sidney, Kelly, Jean e Jaime.

Por fim agradeço meu orientador Orlando Stanley Juriaans, pois o mesmo entende bem o papel do orientador, e fico muito grato pelo direcionamento da tese, e por ter incorporado-me na pesquisa de álgebra de Colombeau. 


\section{Resumo}

As álgebras de funções generalizadas de Colombeau são ambientes naturais em que equações lineares e não lineares podem ser tratadas. O objetivo desta tese é continuar desenvolvendo a teoria das funções generalizadas de Colombeau, assim como construir novas álgebras diferenciais em que as distribuições de Schwartz podem ser mergulhadas.

No primeiro capítulo, damos uma introdução desta teoria e relatamos os principais resultados que serão imporantes para o desenvolvimento desta tese. As provas de muitos resultados são incluídas para a facilidade do leitor e para fazer o texto mais acessível.

No segundo capítulo, estudamos os ideiais maximais da álgebra plena de funções generalizadas de Colomebau e classificamos completamente estes ideiais maximais. Introduzimos as álgebras de Aragona e usamos um processo de compactificação, similiar à de Stone Check, introduzida por Khelif - Scarpalezos para conseguir esta classificação. Em particular, completamos a classificação de Khelif - Scarpalezos obtida para as álgebras simplificadas. Este capítulo serve também de base para boa parte da tese.

No capítulo 3, generalizamos as noções de conjuntos internos e membranas, introduzidas por Aragona - Fernandes -Juriaans -Oberguggenberger - Vernaev, para o contexto da álgebra plena. Vários resultados então generalizados para o contexto da álgebra plena.

Se $f \in \mathcal{H}(\Omega)$ é uma função holomorfa clássica, $R_{f} \subset \mathbb{C}$ o conjunto de zeros da $f$ e

$\mathcal{G}_{f} \mathcal{R}_{f} \subset \overline{\mathbb{K}}$ o conjunto de zeros generalizados da $f$, começamos relacionando estes dois conjuntos usando as teorias mais recentes desenvolvidas na áreas. Isto é feito no capítulo 4. Palavras-chave: Funções Holomorfa generalizadas, Conjunto Interno, Álgebra de Aragona. 


\section{Abstract}

Colombeau generalized functions are natural environment in which linear and nonlinear relationship can be treated. This $\mathrm{PhD}$ thesis aims to continue the developing the Colombeau theory of generalized functions, as well as building new differential algebras in order to use a monomorphism with Schwartz distributions can be dipped. The first chapter of this thesis brings an introduction to the Colombeau theory and describes the main results that have been obtained in the literature, which are important for the development, we describe the main results - which are important for the development of this thesis. In order to help the reader and to make the text more accessible, mathematical evidence of these results are also presented in this chapter. In the second chapter, it was studied the maximal ideal of full Colombeau algebra of generalized functions and completely classified these ideals. We introduced the Aragona algebras and used a compactification process similar to Stone - Check, which was introduced by Khelif - Scarpalezos to obtain this classification. In particular, we completed a classification of Khelif - Scarpalezos that is applied for simplified algebras. This chapter also serves as the basis for this thesis. In chapter 3, we generalize the internal sets and membranes, introduced by Aragona - Fernandes -Juriaans -Oberguggenberger - Vernaev, for the context of full algebra. Several results are then generalized to the context of full algebra.

If $f \in \mathcal{H}(\Omega)$ is a classic holomorphic function, $R_{f} \subset \mathbb{C}$ the zero set of $f$ and $\mathcal{G}_{f} \mathcal{R}_{f} \subset \overline{\mathbb{K}}$ the set of generalized zeros from $f$, we then related these two sets using the latest theories developed in this field and this work is presented in the Chapter 4. 


\section{Sumário}

Lista de Símbolos $\quad$ xi

Lista de Símbolos $\quad$ xiii

1 Introdução $\quad 1$

1.1 Preliminares: A Álgebra Simplificada $\mathcal{G}(\Omega) \ldots \ldots$. . . . . . . . . . . . 2

1.2 A Álgebra Full $\mathcal{G}_{f}(\Omega) \ldots \ldots \ldots \ldots \ldots$. . . . . . . . . . . . . . 4

1.2.1 Definições e Propriedades . . . . . . . . . . . . . . . . . . 4

1.2.2 A imersão de $D^{\prime}(\Omega)$ em $\mathcal{G}_{f}(\Omega) \ldots \ldots \ldots$. . . . . . . . . 6

1.2.3 Pontos Generalizados . . . . . . . . . . . . . . . . . . 8

1.2.4 Alguns Resultados na Álgebra $\mathcal{G}_{f}(\Omega) \ldots \ldots$. . . . . . . . . . . . 10

1.3 Topologias Naturais Sobre as Álgebras de Colombeau em $\mathcal{G}_{f}(\Omega)$. . . . . . . 11

1.3.1 A topologia Sharp sobre $\mathcal{G}_{f}(\Omega) \ldots \ldots \ldots \ldots 11$

1.3.2 Propriedades de $\overline{\mathbb{K}}_{f} \ldots \ldots \ldots \ldots$. . . . . . . . . . . . . . . . . . . . . . . . . . . . . . . 13

1.3.3 Cálculo Diferencial sobre $\overline{\mathbb{K}}^{n} \ldots \ldots \ldots \ldots$. . . . . . . . . 15

1.4 Análise Não Standard . . . . . . . . . . . . . . . . . . . . . . . 16

1.4.1 Elementos multiplicativos . . . . . . . . . . . . . . . . 19

1.5 Compactificação do $X(n, \mathbb{L}) \ldots \ldots \ldots$. . . . . . . . . . . . 20

2 Álgebras de Aragona $\quad 25$

2.1 Preliminar . . . . . . . . . . . . . . . . . . 25

2.2 Álgebras de Aragona . . . . . . . . . . . . . . . . . . . . 26

2.3 Filtros e Idempotentes . . . . . . . . . . . . . . . . . . . . . . . . . . 32

2.4 Traços de Ideais . . . . . . . . . . . . . . . . . . . . . . . . 33

3 Conjuntos Internos $\quad 37$

3.1 Teoria dos Conjuntos Internos . . . . . . . . . . . . . . . . . . 37

3.2 Funções Internas . . . . . . . . . . . . . . . . . . . . . . . . . . 44

3.3 Aplicações . . . . . . . . . . . . . . . . . . . . . . . . . . . 46

4 Zeros de Funções Holomorfas Generalizados $\quad 51$ 
5 Conclusões

5.1 Sugestões para Pesquisas Futuras . . . . . . . . . . . . . . . . . 53

Referências Bibliográficas 


\section{Lista de Símbolos}

1. $\mathbb{N}_{0}$ é o conjunto $\mathbb{N} \cup\{0\}$

2. $I:=] 0,1], \bar{I}:=[0,1]$ e $\left.I_{\eta}:=\right] 0, \eta[, \forall \eta \in I$

3. $A / B:=\{a \in A \mid a \notin B\}$

4. $\mathbb{Q}$ denotará o corpo dos números racionais.

5 . $\mathbb{K}$, como é usual, denortará o corpo real(ou, complexo), i.e, $\mathbb{R}$ ou $\mathbb{C}$.

6. $\mathbb{N}, \mathbb{Z}$ denotará respectivamente, o conjunto dos números naturais e o conjunto dos números inteiros.

7. $\mathbb{N}^{*}:=\mathbb{N} /\{0\}$ e $\mathbb{Z}^{*}:=\mathbb{Z} /\{0\}$

8. $\mathbb{K}^{*}:=\mathbb{K} /\{0\}$

9. $\mathbb{R}_{+}:=\{x \in \mathbb{R} \mid x \geq 0\}$ e $\mathbb{R}_{+}^{*}:=\{x \in \mathbb{R} \mid x>0\}$

10. $\Omega$ é um subconjunto aberto, não vazio, de $\mathbb{R}^{n}$ e $K \subset \subset \Omega$ sifnifica que $K$ é um subconjunto compacto de $\Omega$. Dada uma função $f: X \rightarrow \mathbb{K}$ e $\emptyset \neq Y \subset X$ definimos $\|f\|_{Y}:=\sup _{x \in K}|f(x)|$.

11. $\mathbb{N}^{n}:=\underbrace{\mathbb{N} \times \ldots \times \mathbb{N}}_{\mathrm{n} \text { vezes }}$. Um elemento $\alpha \in \mathbb{N}^{n}$ é chamado multi-índice.

12. Temos $|\alpha|=\sum_{i=1}^{n} \alpha_{i}$ e $\partial^{\alpha}:=\frac{\partial^{|\alpha|}}{\partial_{x_{1}}^{\alpha} \ldots \partial_{x_{n}}^{\alpha_{n}}}$

13. Fórmula de Leibniz: $\partial^{\alpha}(u \cdot v)=\sum_{0 \leq \beta \leq \alpha}\left(\begin{array}{c}\alpha \\ \beta\end{array}\right) \partial^{\alpha-\beta} u \partial^{\beta} v$, onde $\alpha, \beta \in \mathbb{N}^{n}, \beta=\left(\beta_{1}, \ldots, \beta_{n}\right) \mathrm{e}$ $\alpha=\left(\alpha_{1}, \ldots, \alpha_{n}\right)$ generalização tal que $\beta \leq \alpha \Leftrightarrow \beta_{i} \leq \alpha_{i}, \forall i=1,2, . ., n$.

14. $\overline{\mathbb{K}}$ denotará o anel dos números generalizados simplificado de Colombeau.

15. $\overline{\mathbb{R}}$ denotará o anel dos números generalizados simplificadode Colombeau sobre o corpo dos números dos reais.

16. $\overline{\mathbb{C}}$ denotará o anel dos números generalizados simplificado de Colombeau sobre o corpo dos números complexos.

17. $\overline{\mathbb{K}}_{f}$ denotará o anel dos números generalizados pleno de Colombeau.

18. $\overline{\mathbb{R}}_{f}$ denotará o anel dos números generalizados pleno de Colombeau sobre o corpo dos números reais.

19. $\overline{\mathbb{C}}_{f}$ denotará o anel dos números generalizados pleno de Colombeau sobre o corpo dos números complexos.

20. $\mathcal{A}_{0}(\mathbb{R}):=\left\{\varphi \in \mathcal{D}(\mathbb{R}) \mid \int_{0}^{\infty} \varphi(x) d x=\frac{1}{2}, \varphi\right.$ é par e $\varphi \equiv$ const. em $\left.V_{0}\right\}$ onde $V_{0}$ é uma vizinhaça da origem. 


\section{Lista de Símbolos}

21. $\mathcal{A}_{q}(\mathbb{R}):=\left\{\varphi \in \mathcal{A}_{0}(\mathbb{R}) \mid \int_{0}^{\infty} x^{\frac{j}{m}} \varphi(x) d x=0\right.$, para $\left.1 \leq j, m \leq q\right\}$ onde $q \in \mathbb{N}$.

22. $\mathbb{K}^{*}:=\mathbb{K} /\{0\}$.

23. $\Gamma\left\{\gamma: \mathbb{N} \rightarrow \mathbb{R}_{+} \mid \gamma(n+1), \forall n \in \mathbb{N}\right.$ e $\left.\lim _{n \rightarrow \infty} \gamma(n)=\infty\right\}$ é o conjunto das sequências estritamente crescente que divergem quando $n \rightarrow \infty$

24. $\mathcal{G}(\Omega)$ denotará a álgebra das funções generalizadas simplificada de Colombeau.

25. $\mathcal{G}_{C}(\Omega)$ denotará a álgebra das funções generalizadas simplificada de Colombeau de suporte compacto.

26. $\mathcal{G}_{f}(\Omega)$ denotará a álgebra das funções generalizadas plena de Colombeau.

27. $\mathcal{G}_{C C}(\Omega)$ denotará a álgebra das funções generalizadas plena de Colombeau com suporte compacto.

28. $\mathcal{D}(\Omega)$ denotará o espaço das funções $C^{\infty}(\Omega)$ com suporte comapcto em $\Omega \subset \mathbb{R}^{n}$.

29. $\mathcal{D}^{\prime}(\Omega)$ denotará o espaço das distribuições.

30. $\mathcal{A}(m, \mathbb{L})$ a Álgebra de Aragona com $m \triangleleft \bar{K}_{f}$ um ideal maximal e $\mathbb{L}$ um corpo.

31. $\mathcal{M}$ ideais maximais na algebra plena de Colombeau.

32. $\gamma(X(n, \mathbb{L}))$ é a compactificação do espaço de Aragona. 


\section{Capítulo 1}

\section{Introdução}

Laurent Schwartz está entre os primeiros a criar uma teoria de funções generalizadas para lidar com a solução de equações diferenciais. Porém, a sua teoria é uma teoria linear, ou seja, pode ser usada para resolver equações diferenciais lineares. Schwartz também provou que se $\Omega \subset \mathbb{R}^{n}$ é um aberto então não existe uma álgebra diferencial associativa $A$ contendo $D^{\prime}(\Omega)$ de forma que $C(\Omega)$ seja uma subálgebra de $A$ e tal que a derivação em $D^{\prime}(\Omega)$ seja a restrição da derivação de $A$. Este resultado é conhecido como o Teorema de Impossibilidade de Schwartz. No entanto, equações não lineares que modelam fenómenos reais são abundantes sugerindo que deveria existir um ambiente diferencial e algébrico que contém $D^{\prime}(\Omega)$. Muitas propostas para multiplicação de distribuições, em casos particulares, foram feitas. Por outro lado, muitos matemáticos, a pesar do Teorema de Impossibilidade de Schwartz, continuaram acreditando na existência de um ambiente algébrico e diferencial que contém $D^{\prime}(\Omega)$. Um dos principais pesquisadores que perseguiu esta ideia, foi o matemático Rossinger. Mas coube ao matemático J.F. Colombeau, na dêcada de oitenta, construir uma álgebra diferecial $\mathcal{G}(\Omega)$ que contém uma cópia linear de $D^{\prime}(\Omega)$, cuja derivação estende a de $D^{\prime}(\Omega)$ e na qual $C^{\infty}(\Omega)$ pode ser imersa como uma subálgebra. Observe que isto não contradiz o Teorema de Impossibilidade de Schwartz. A álgebra diferencial $\mathcal{G}(\Omega)$ é chamada a álgebra de funções generalizadas de Colombeau.

Existem muitas equações lineares, muito simples, que não podem ser resolvidas usando a Teoria de Schwartz; nem solubilidade local pode ser provado. Porém, em $\mathcal{G}(\Omega)$ qualquer diferencial linear tem uma solução!

Desde então, a teoria de funções generalizadas de Colombeau vem sendo desenvolvida e já existem aplicações na teoria de equações diferenciais lineares e não linear, geometria diferencial e física matemática. Uma das dificuldades porém era que a teoria não era intrínsica. Havia, portanto, a necessidade para desenvolver as partes algébricas, topológicas e analíticas da teoria. A parte álgebrica começou a ser desenvolvida por Aragona - Juriaans num artigo pioneira na área. Dado um elemento $f \in \mathcal{G}(\Omega)$ e $x \in \Omega$ podemos definir $f(x)$ que pertence a $\overline{\mathbb{K}}$ que é chamado o anel de números generalizados de Colombeau. Este anel, faz o papel que $\mathbb{R}$ faz no caso do estudo de funções a valores reais. Aragona - Juriaans estudaram as propriedades álgebricas e topológicas de $\overline{\mathbb{K}}$. Por outro lado, Kunzinger - Oberguggenberger associaram a $\Omega$ um subconjunto $\widetilde{\Omega}_{c} \subset \overline{\mathbb{K}}^{n}$ de forma que um elemento $f \in \mathcal{G}(\Omega)$ é nulo se e somente se $f(x)=0, \forall x \in \widetilde{\Omega}_{c}$. Com isto os elementos de $\mathcal{G}(\Omega)$ se tornam funções no sentido clássico e distribuições podem ser vistas como funções no sentido clássico.

No artigo [AFJO12], Aragona - Fernandes - Juriaans introduziram o calculo diferencial no cenário das álgebras de Colombeau. Com isto, a teoria de Colombeau se torna uma extenção natural do calculo diferencial clássico. Posteriormente, eles introduziram a noção de membranas $n$-dimensional e definem integração sobre membranas. Eles também provam al- 
gumas propriedades de membranas como por exemplo, que todas as membranas são fechadas na topologia Sharp, e desenvolvem ferramentas de diferenciabiliade.

Estendendo a teoria sobre membranas, Oberguggenberger e Vernaeve em [OV08] introduziram os conjuntos internos, uma ferramenta natural na Análise não standard. Neste artigo [OV08] eles definem $\mathcal{G}_{E}$ (onde a topologia de $E$ é gerada por uma família de semi-normas) e chamaram de espaço de Colombeau baseado em $E$. No caso quando $E=\mathbb{R}$ escrevemos $\widetilde{R}$ e $\widetilde{\mathbb{C}}$ para $\mathcal{G}$. Quando $\Omega$ é um aberto de $\mathbb{R}^{d}$ e $E=C^{\infty}(\Omega)$, o espaço $\mathcal{G}$ é a álgebra diferencial de funções generalizadas de Colombeau, $\mathcal{G}(\Omega)$. Os conjuntos internos são objetos mais gerais do que membranas. Os autores também definiram funções internas, e provaram que conjuntos internos apresentão varias propriedades úteis, como por exemplos são fechados na topologia Sharp e funções internas que são uniformemente continuas na topologia Sharp. Provaram um Princípio da Saturacão, no contexto das Álgebras de Colombeau, e fizeram varias aplicações para este princípio.

Neste trabalho, desenvolvemos a teoria de conjuntos internos para a álgebra Full de Colombeau, denotado por $\mathcal{G}_{f}(\Omega)$. Utilizando a definição de $\widetilde{\Omega}$ e $\widetilde{\Omega}_{c}$, introduzida na álgebra Full de Colombeau em [Nig13], definimos conjuntos internos em $\widetilde{\Omega}$ e, utilizando algumas técnicas da álgebra Full de Colombeau introduzidas em [Nig13] e [Gar06], provamos várias propriedades de conjuntos internos, estendemos o Princípio da Saturação e fazemos aplicações na álgebra Full de Colombeau. No capitulo 4, provamos que os zeros de uma função holomorfa clássica $f \in \mathcal{H}(\Omega)$ está relacionados com os zeros generalizados, desta mesma função, via a teoria dos conjuntos internos.

No capitulo 2, motivado na construção de Colombeau que mergulha o $D^{\prime}(\Omega)$ em $\mathcal{G}_{f}(\Omega)$, construímos novas álgebras, chamadas Álgebras de Aragona e assim como nas álgebras de Colombeau, mergulhamos $D^{\prime}(\Omega)$ nas Álgebras de Aragona. Mais geralmente, construímos um homomorfismo de $\mathcal{G}(\Omega)$ numa Álgebras de Aragona. Denotando uma álgebra de Aragona por $\mathcal{A}(\Omega)$, vejamos como os ambientes algébricos, construídos até agora, se relacionam com as Álgebras de Colombeau e com as álgebras de Aragona:

$$
\begin{gathered}
\mathbb{N} \subset \mathbb{Z} \subset \mathbb{Q} \subset \mathbb{R} \subset \mathbb{C} \subset C^{\infty}(\Omega) \subset C^{k}(\Omega) \subset C(\Omega) \subset L_{l o c}^{p}(\Omega) \subset D^{\prime}(\Omega) \subset \mathcal{A}(\Omega) \\
0 \rightarrow J \rightarrow \mathcal{G}_{f}(\Omega) \rightarrow \mathcal{A}(\Omega) \rightarrow 0
\end{gathered}
$$

O ponto central de todas as construções é a Álgebra de Colombeau. Provamos que $J \triangleleft$ $\mathcal{G}_{f}(\Omega)$ é um ideal diferencial. Consequentemente, o Cálculo Diferencial desenvolvido por Aragona - Fernandes -Juriaans em $\mathcal{G}(\Omega)$ induz um Cálculo Diferencial em $\mathcal{A}(\Omega)$. Além do mais $J \cap D^{\prime}(\Omega)=\{0\}$ e existe um corpo $\mathbb{L}$ e um espaço últramétrica $X$, chamado de espaço de Aragona, tal que $\mathcal{A}(\Omega)=\mathcal{F}(X, \mathbb{L})$. Usamos este Cálculo Diferencial nas Álgebas de Aragona, em conjunto com um processo de compactificação desenvolvido por Khelif - Scarpalezos, para dar uma classificação completa dos ideiais maximais de $\mathcal{G}_{f}(\Omega)$. Khelif - Scarpalezos conseguiram uma classificação parcial no caso da ágebra simplicada e nos mandaram o seu artigo quando os nossos resultados já estavam prontos.

\subsection{Preliminares: A Álgebra Simplificada $\mathcal{G}(\Omega)$}

A teoria de distribuições, fundamentada por S.L. Sobolev e L. Schwartz, é muito útil na teoria de equações diferênciais lineares. A tentativa de se definir uma operação sobre produtos $f \cdot g,(f, g) \in D^{\prime}(\Omega) \times D^{\prime}(\Omega)$, levam a contradições: 
Suponhamos que exista uma álgebra diferencial comutativa e associativa $D^{\prime}(\Omega) \subset A$ cuja derivação, quando restrita, coincide com a de $D^{\prime}(\Omega)$. Considere a função de Heaviside $H \in D^{\prime}(\Omega)$. O fato de $H^{2}=H \cdot H=H$, será usado para provar que $H^{\prime}=0$, uma contradição. De fato, $\left(H^{2}\right)^{\prime}=H^{\prime} H+H H^{\prime}=2 H H^{\prime}$ e $\left(H^{3}\right)^{\prime}=3 H^{2} H^{\prime}$. Como $H=H^{2}=H^{3}$, tem-se que $2 H H^{\prime}=H^{\prime}=3 H H^{\prime}$. Portanto, $H H^{\prime}=0$ e, consequentemente, $H^{\prime}=0$, uma contradição pois $H^{\prime}=\delta \neq 0$.

Queremos uma álgebra diferencial $A(\Omega)$ em que podemos mergulhar $\mathcal{D}^{\prime}(\Omega)$ linearmente, ou seja, queremos uma álgebra $(\mathcal{A}(\Omega),+, \circ)$ associativa e comutativa satisfazendo:

(i) $\mathcal{D}^{\prime}(\Omega)$ é mergulhado linearmente dentro $\mathcal{A}(\Omega)$, e $f(x) \equiv 1$ é a unidade em $\mathcal{A}(\Omega)$.

(ii) Existem derivações $\partial_{i}: \mathcal{A}(\Omega) \rightarrow \mathcal{A}(\Omega),(i=1, \ldots, n)$ que satisfazem a regra de Leibniz.

(iii) $\left.\partial_{i}\right|_{\mathcal{D}^{\prime}}(\Omega)$ é a derivada parcial usual $(i=1, \ldots, n)$ em $\mathbb{R}^{n}$.

(iv) $\left.\circ\right|_{L_{l o c}^{\infty}(\Omega) \times L_{l o c}^{\infty}(\Omega)}$ coincide com o produto de funções ponto a ponto.

Álgebras satisfazendo ( $i i)$ são chamadas álgebras diferênciais, porém, em qualquer álgebra associativa e comutativa, satisfazendo $(i),(i i),(i i i)$ e $(i v)$ são mutualmente excludente. O leitor pode consultar o [Kun96] para mais detalhes. Com isso em mente deixamos o item (iv) de lado e pedimos que satisafaça o seguinte item:

$\left.(v) \circ\right|_{C(\Omega) \times C(\Omega)}$ coincide com produtos de funções ponto a ponto.

O resultado abaixo é o Teorema de Impossibilidade de Schwartz.

Teorema 1.1.1. Não existe uma álgebra comutativa, associativa $(\mathcal{A}(\Omega),+, \circ)$ satisfazendo (i), (ii), (iii) e (v).

Demonstração. Consulte [Kun96].

J.F. Colombeau prova no entanto que existe uma álgebra asssociativa e comutativa satisfazendo $(i),($ ii $),($ iii $)$ e também;

(vi) $\left.\circ\right|_{C^{\infty}(\Omega) \times C^{\infty}(\Omega)}$ coincide como produto de funções ponto a ponto.

O leitor pode consultar [Col82] e [Kun96]. A seguir, detalhamos a sua construção.

Definição 1.1.2. Seja $\Omega \subset \mathbb{R}^{n}$ um aberto e $\mathcal{E}(\Omega):=\left(C^{\infty}(\Omega)\right)^{I}$, onde $I=(0,1]$.

$\mathcal{E}_{M}(\Omega):=\left\{\left(u_{\varepsilon}\right)_{\varepsilon} \in \mathcal{E}(\Omega): \forall K \subset \subset \Omega, \forall \alpha \in \mathbb{N}_{0}^{n}, \exists p \in \mathbb{N}, \quad\right.$ tal que $\sup _{x \in K}\left|\partial^{\alpha} u(\varepsilon, x)\right|=$ $\left.O\left(\varepsilon^{-p}\right)(\varepsilon \rightarrow 0)\right\}$

$\mathcal{N}(\Omega):=\left\{\left(u_{\varepsilon}\right)_{\varepsilon} \in \mathcal{E}(\Omega): \forall K \subset \subset \Omega, \forall \alpha \in \mathbb{N}_{0}^{n}, \forall q \in \mathbb{N}, \quad\right.$ tal que $\sup _{x \in K}\left|\partial^{\alpha} u(\varepsilon, x)\right|=$ $\left.O\left(\varepsilon^{q}\right)(\varepsilon \rightarrow 0)\right\}$

Observe que $\mathcal{E}_{M}(\Omega)$ é uma álgebra diferencial e $\mathcal{N}(\Omega) \triangleleft \mathcal{E}_{M}(\Omega)$ é um ideal diferencial. A álgebra de Colombeau! $\mathcal{G}(\Omega)$ é o quociente $\mathcal{G}(\Omega)=\mathcal{E}_{M}(\Omega) / \mathcal{N}(\Omega)$ e logo é também uma álgebra diferencial comutativa e associativa.

Os elementos $u_{\varepsilon}$ na definição acima podem tomar valores reais ou complexas e para distinguir isto escrevemos $\mathcal{G}(\Omega, \mathbb{R})$ ou $\mathcal{G}(\Omega, \mathbb{C})$ ou simplesmente por $\mathcal{G}(\Omega)$ quando o contexto não deixar dúvidas de qual se trata. Denotaremos os elementos de $\mathcal{G}(\Omega)$ por letras maúsculas e seus representantes por letras minúsculas, ou seja, $U=\left[\left(u_{\varepsilon}\right)_{\varepsilon}\right]$. Nada porém nos impede para escrever $u=\left[\left(u_{\varepsilon}\right)_{\varepsilon}\right]$. É facil ver que $C^{\infty}(\Omega)$ é uma subálgebra fiel de $\mathcal{G}(\Omega)$ através da imersão diagonal $\sigma: f \rightarrow\left[\left(f_{\varepsilon}=f\right)_{\varepsilon}\right]$.

A álgebra que acabamos de definir acima é conhecida como a álgebra simplificada. Para esta tese estaremos interessados na álgebra full ou plena. A sua definição é bem mais envolvente e é isto que veremos a seguir. 


\section{$1.2 \quad$ A Álgebra Full $\mathcal{G}_{f}(\Omega)$}

\subsubsection{Definições e Propriedades}

Nosso objetivo é introduzir a álgebra de funções generalizadas conhecida como álgebra full ou plena de funções generalizadas de Colombeau e denotado por $\mathcal{G}_{f}$. A abordagem que seguiremos é devido a Aragona - Biagioni. Esta abordagem é mais intrínsica (vide [AB91a]).

\section{Definição 1.2.1.}

$$
\begin{aligned}
& \mathcal{A}_{0}(\mathbb{R}, \mathbb{K}):=\left\{\varphi \in D(\mathbb{R}, \mathbb{K}): \int_{0}^{\infty} \varphi(x) d x=\frac{1}{2}, \varphi \text { é par, } \varphi \text { é const. em vizinhança do } 0\right\} \\
& \mathcal{A}_{q}(\mathbb{R}, \mathbb{K}):=\left\{\varphi \in \mathcal{A}_{0}(\mathbb{R}, \mathbb{K}): \int_{0}^{\infty} x^{\frac{j}{m}} \varphi(x) d x=0, \text { para } 1 \leq j, m \leq q\right\}(q \in \mathbb{N}) .
\end{aligned}
$$

Os elementos de $D(\mathbb{R}, \mathbb{K})$ podem ter valores real ou complexo, e obtemos os conjuntos $\mathcal{A}_{q}(\mathbb{R} ; \mathbb{R})$ ou $\mathcal{A}_{q}(\mathbb{R} ; \mathbb{C})$, respectivamente. Mas, não nos preocupamos com essa diferença, porém quando $\mathbb{K}=\mathbb{R}$, temos o seguinte resultado:

Proposição 1.2.2. O conjunto $\mathcal{A}_{q}(\mathbb{R} ; \mathbb{R})=\mathcal{A}_{q}(\mathbb{R})$ é não-vazio para cada $q \in \mathbb{N}^{*}$. Mais precisamente, para $a, b \in \mathbb{R}_{+}$e para cada $q \in \mathbb{N}^{*}$, existe $\varphi_{1} \in \mathcal{A}_{q}(\mathbb{R})$ tal que $\varphi_{1}(a)=b$.

Demonstração. Vide [AB91a].

Com os conjuntos $\mathcal{A}_{q}(\mathbb{R})$ podemos construir classes de funções testes conhecida como classes de mollifiers para espaços normados suaves de dimensão finita. Um espaço normado $E$ é dito suave, se $E$ é um espaço vetorial normado de dimensão finita que é diferenciável sobre $E-\{0\}$. Para $n \in \mathbb{N}$, denotamos por $\omega_{n}$ a área da esfera $(n-1)-$ dimensional $S^{n-1}$ em $\mathbb{R}^{n}$ e definimos

$$
c_{n}:=\left\{\begin{array}{lll}
\frac{2 n}{\omega_{n}}, & \text { para } & n>1 \\
1 & \text { para } & n=1
\end{array}\right.
$$

Se $E$ é um espaço normado suave $n$-dimensional(escrevemos $E=E_{n}$ ) com norma $\|\cdot\|_{E_{n}}$, então para cada $\varphi_{1} \in \mathcal{A}_{0}(\mathbb{R})$ a aplicação $\varphi: x \rightarrow c_{n} \varphi_{1}\left(\|x\|_{E_{n}}^{n}\right) \in D\left(E_{n}\right)$ está em $D(E)$ e pode ser provado que

$$
I_{E}^{1}=I_{E_{n}}^{1}: \varphi_{1} \in \mathcal{A}_{0}(\mathbb{R}) \rightarrow \varphi=c_{n}\left(\varphi_{1} \circ\|\cdot\|_{E_{n}}^{n}\right) \in D\left(E_{n}\right)
$$

define uma aplicação injetora.

Definição 1.2.3. Para todo espaço normado suave $E_{n}$ e para todo $q \in \mathbb{N}$ definimos

$$
\mathcal{A}_{q}\left(E_{n}\right):=I_{E_{n}}^{1}\left(\mathcal{A}_{q}(\mathbb{R})\right) .
$$

O próximo resultado será muito útil em vários momentos durante o trabalho desta tese.

Proposição 1.2.4. Se $E=E_{n}$ é um espaço normado suave $\varphi \in \mathcal{A}_{q}\left(E_{n}\right)$ e $P \in \mathcal{P}_{k}(E, \mathbb{K})(o$ espaço dos polinômios $k$ - homogêneos sobre $E$ ) com $1 \leq k \leq q$, então

$$
\int_{E_{n}} \varphi(x) d x=1 \text { e } \int_{E_{n}} P(x) \varphi(x) d x=0, \text { para } q \geq n .
$$


Demonstração. Vide [Kun96].

Listamos agora as propriedades dos conjuntos $\mathcal{A}_{q}$. Seja a aplicação $I_{F}^{E}:=I_{F}^{1} \circ I_{1}^{E}$, temos o seguinte resultado.

Proposição 1.2.5. Se $E=E_{n}$ é um espaço normado suave, então existe uma sequência $\left(\mathcal{A}_{q}(E)\right)_{q=0}^{\infty}$ de conjuntos satisfazendo

(i) $\emptyset \neq \mathcal{A}_{q+1}(E) \subseteq \mathcal{A}_{q}(E) \subseteq D(E) \forall q \in \mathbb{N}_{0}$.

(ii) $\mathcal{A}_{q+1}(E ; \mathbb{R}) \subseteq \mathcal{A}_{q+1}(E ; \mathbb{C}) \forall q \in \mathbb{N}_{0}$

(iii) Se $q \in \mathbb{N}_{0}, \varphi \in \mathcal{A}_{q}(E)$ e $\varepsilon>0$ então $\varphi_{\varepsilon} \in \mathcal{A}_{q}(E)$.

(iv) Para cada $x \in E, b \in \mathbb{R}_{+}$e $q \in \mathbb{N}_{0}$ existe algum $\varphi \in \mathcal{A}_{q}(E) \operatorname{com} \varphi(x)=b$.

(v) Para qualquer espaço normado suave $F=F_{m}$, existe uma bijeção

$$
I_{F}^{E}: \mathcal{A}_{0}(E) \rightarrow \mathcal{A}_{0}(F)
$$

que satisfaz as seguintes condições:

(a) $I_{F}^{E}: \mathcal{A}_{q}(E) \rightarrow \mathcal{A}_{q}(F) \forall q \in \mathbb{N}_{0}$

(b) $I_{E}^{E}$ é a aplicação identidade sobre $\mathcal{A}_{0}$

(c) $I_{F}^{E}\left(\varphi_{\varepsilon}\right)=\left(I_{F}^{E}(\varphi)\right)_{\varepsilon^{\frac{n}{m}}} \forall \varphi \in \mathcal{A}_{0}(E), \forall \varepsilon>0$.

(d) Se $G=G_{p}$ é um espaço normado suave então $I_{F}^{E}=I_{F}^{G} \circ I_{G}^{E}$.

(vi) Cada $\varphi \in \mathcal{A}_{0}(E)$ é par e constante em uma vizinhança de 0 .

(vii) $\bigcap_{q=0}^{\infty} \mathcal{A}_{q}(E)=\emptyset$

Demonstração. Vide [Kun96].

Para $E=\mathbb{R}^{n}$ e $F=\mathbb{R}^{m}$ usaremos a notação $I_{F}^{E}$ e $\mathcal{A}_{q}(n)$ em vez de $\mathcal{A}_{q}\left(\mathbb{R}^{n}\right)$.

Definição 1.2.6. Para qualquer subconjunto aberto $\Omega$ de um espaço normado suave E, definimos:

$$
\mathcal{E}(\Omega, \mathbb{K})=\left\{u: \mathcal{A}_{0}(E, \mathbb{K}) \times \Omega \rightarrow \mathbb{K}: u(\varphi, .) \in C^{\infty}(\Omega), \forall \varphi \in \mathcal{A}_{0}(E, \mathbb{K})\right\}
$$

$\mathcal{E}_{M, f}(\Omega, \mathbb{K})=\left\{u \in \mathcal{E}(\Omega): \forall K \subset \subset \Omega, \forall n \in \mathbb{N}_{0}, \exists p \in \mathbb{N}_{0}, \forall \varphi \in \in \mathcal{A}_{p}(E, \mathbb{K}), \exists C>0\right.$ e $\exists \eta>$ 0 tal que $\left.\sup _{x \in K}\left\|D^{n}\left(\varphi_{\varepsilon}, x\right)\right\| \leq C \varepsilon^{-p}(0<\varepsilon<\eta)\right\}$

$\mathcal{N}_{f}(\Omega, \mathbb{K})=\left\{u \in \mathcal{E}(\Omega): \forall K \subset \subset \Omega, \forall n \in \mathbb{N}_{0}, \exists p \in \mathbb{N}_{0}, \exists \gamma \in \Gamma, \forall q \geq p, \forall \varphi \in\right.$ $\mathcal{A}_{p}(E, \mathbb{K}), \exists C>0 \quad e \exists \eta>0$ tal que $\left.\sup _{x \in K}\left\|D^{n}\left(\varphi_{\varepsilon}, x\right)\right\| \leq C \varepsilon^{\gamma(q)-p}(0<\varepsilon<\eta)\right\}$

onde $\Gamma=\left\{\gamma: \mathbb{N} \rightarrow \mathbb{R}_{+}: \gamma\right.$ é estritamente crescente, $\left.\lim _{n \rightarrow \infty} \gamma(n)=\infty\right\}$.

É importante notar que os elementos de $\mathcal{A}_{0}(E, \mathbb{K})$ servem como parâmetros na definição acima.

Definição 1.2.7. A álgebra plena de Colombeau é a álgebra diferencial

$$
\mathcal{G}_{f}(\Omega ; \mathbb{K})=\mathcal{E}_{M, f}(\Omega ; \mathbb{K}) / \mathcal{N}_{f}(\Omega ; \mathbb{K})
$$

Se não houver dúvidas sobre o contexto, escreveremos $\mathcal{G}_{f}(\Omega)$ invés de $\mathcal{G}_{f}(\Omega ; \mathbb{K})$. 
Proposição 1.2.8. Se $\Omega$ é um subconjunto aberto de $\mathbb{R}^{n}$, então

$\mathcal{E}_{M, f}(\Omega)=\left\{u \in \mathcal{E}(\Omega): \forall K \subset \subset \Omega, \forall \alpha \in \mathbb{N}_{0}^{n}, \exists p \in \mathbb{N}_{0}, \forall \varphi \mathcal{A}_{p}(n), \exists C>0 \quad\right.$ e $\exists \eta>0$ tal que $\left.\sup _{x \in K}\left\|\partial^{\alpha} u\left(\varphi_{\varepsilon}, x\right)\right\| \leq C \varepsilon^{-p}(0<\varepsilon<\eta)\right\}$,

$\mathcal{N}_{f}(\Omega, \mathbb{K})=\left\{u \in \mathcal{E}(\Omega): \forall K \subset \subset \Omega, \forall \alpha \in \mathbb{N}_{0}^{n}, \exists p \in \mathbb{N}_{0}, \exists \gamma \in \Gamma, \forall q \geq p, \forall \varphi \in \mathcal{A}_{p}(n), \exists C>\right.$ 0 e $\exists \eta>0$ tal que $\left.\sup _{x \in K}\left\|\partial^{\alpha} u\left(\varphi_{\varepsilon}, x\right)\right\| \leq C \varepsilon^{\gamma(q)-p}(0<\varepsilon<\eta)\right\}$.

Vejamos agora algumas propriedades básicas destas novas álgebras.

Teorema 1.2.9. $\mathcal{G}_{f}$ é um feixe fino de álgebras diferêncial sobre $\mathbb{R}^{n}$.

\subsubsection{A imersão de $D^{\prime}(\Omega)$ em $\mathcal{G}_{f}(\Omega)$}

Nesta seção veremos que existe uma imersão linear canônica de $\left.D^{\prime} \Omega\right)$ em $\mathcal{G}_{f}(\Omega)$. Iniciamos com o caso especial quando $\Omega=\mathbb{R}^{n}$ e omitiremos as demonstrações. O leitor interessado pode consultar em especial a tese de doutorado de Michael Kunzinger ([Kun96]) citada nas referências.

Teorema 1.2.10. A aplicação

$$
\begin{aligned}
& \iota: \mathcal{D}^{\prime}\left(\mathbb{R}^{n}\right) \rightarrow \mathcal{G}_{f}\left(\mathbb{R}^{n}\right) \\
& w \rightarrow\left[(w * \varphi)_{\varphi \in \mathcal{A}_{0}(n)}\right]
\end{aligned}
$$

é linear e injetivo.

Denotando por $\sigma$ a aplicação $f \rightarrow\left[(f)_{\varphi}\right]$ de $C^{\infty}(\Omega)$ dentro de $\mathcal{G}_{f}(\Omega)$ tem-se:

Proposição 1.2.11. As aplicações $\iota$ e $\sigma$ coincidem sobre $C^{\infty}\left(\mathbb{R}^{n}\right)$.

A ideia de imergir $D^{\prime}(\Omega)$ em $\mathcal{G}_{f}(\Omega)$ é o seguinte: primeiramente mostramos como imergir $\mathcal{E}^{\prime}(\Omega)$, as distribuições de suporte compacto, em $\mathcal{G}_{f}(\Omega)$ e a seguir usamos o fato de $\mathcal{G}_{f}(\Omega)$ ser um feixe fino para construir uma imersão de $D^{\prime}(\Omega)$ em $\mathcal{G}_{f}(\Omega)$. O primeiro passo é relatado no resultado a seguir.

Proposição 1.2.12. Para qualquer $\Omega$ subconjunto aberto de $\mathbb{R}^{n}$, a aplicação

$$
\begin{gathered}
\iota_{0}: \mathcal{E}^{\prime}(\Omega) \rightarrow \mathcal{G}_{f}(\Omega) \\
w \rightarrow\left[\left(\left.(w * \varphi)\right|_{\Omega}\right)_{\varphi \in \mathcal{A}_{0}(n)}\right]
\end{gathered}
$$

é linear e injetivo.

Para o segundo passo definimos uma exaustão de $\Omega$ de subconjuntos compactos de $\Omega, r>$ 0 :

$$
\Omega_{r}:=\left\{x \in \Omega:|x| \leq \frac{1}{r} \text { e } \operatorname{dist}(x, \partial \Omega) \geq 3 r\right\} .
$$

Então $\Omega_{r}$ é compacto, $\Omega_{r} \subseteq \Omega_{s}$ para $s<r$ e

$$
\Omega=\bigcup_{r>0} \Omega_{r}
$$

Denotamos por $\chi_{r}$ a função caracteristica de $\Omega_{r}$ e para $\varphi \in \mathcal{A}_{0}(n)$, consideramos $l(\varphi):=$ $\sup \{|x|: \varphi(x) \neq 0\}$. Então $l\left(\varphi_{\varepsilon}\right)=\varepsilon l(\varphi)$ e, sendo $\operatorname{diam}(\operatorname{supp}(\varphi)) \leq 2 l(\varphi)$, temos

$$
\theta(\varphi):=\chi_{l(\varphi)} * \varphi \in D(\Omega)
$$

Definamos 


$$
\begin{gathered}
\iota: \mathcal{D}^{\prime}(\Omega) \rightarrow \mathcal{G}_{f}(\Omega) \\
w \rightarrow\left[\left(\left.(\theta(\varphi) w * \varphi)\right|_{\Omega}\right)_{\varphi \in \mathcal{A}_{0}(n)}\right]
\end{gathered}
$$

A construção de um mergulho de $\mathcal{D}^{\prime}(\Omega)$ em $\mathcal{G}(\Omega)$ será executado de vários passos. Escolhemos primeiro uma cobertura aberta $\left(\Omega_{\lambda}\right)_{\lambda \in \Lambda}$ de $\Omega$ tal que cada $\bar{\Omega}_{\lambda}$ é um subconjunto compacto de $\Omega$. Seja $\left(\psi_{\lambda}\right)_{\lambda}$ uma família de elementos de $\mathcal{D}(\Omega)$ com $\psi_{\lambda} \equiv 1$ em alguma vizinhança de $\bar{\Omega}_{\lambda}$. Para cada $\lambda \in \Lambda$ definimos,

$$
\begin{gathered}
\iota_{\lambda}: \mathcal{D}^{\prime}(\Omega) \rightarrow \mathcal{G}\left(\Omega_{\lambda}\right) \\
w \rightarrow \iota_{\lambda}(w):=\left(\left.\left(\left(\psi_{\lambda} w\right) * \rho_{\varepsilon}\right)\right|_{\Omega_{\lambda}}\right)_{\varepsilon}+\mathcal{N}\left(\Omega_{\lambda}\right) .
\end{gathered}
$$

A ideia dessa definição é o uso de funções cutoff, então aplicamos a construção anteriormente do mergulho de $\mathcal{E}^{\prime}$.

Proposição 1.2.13. Para qualquer $w \in \mathcal{D}^{\prime}(\Omega),\left(\iota_{\lambda}(w)\right)_{\lambda \in \Lambda}$ é uma família coerente, ou seja,

$$
\left.\iota_{\lambda}(w)\right|_{\Omega_{\lambda} \cap \Omega_{\mu}}=\left.\iota_{\mu}(w)\right|_{\Omega_{\lambda} \cap \Omega_{\mu}}, \forall \lambda, \mu \in \Lambda .
$$
linear.

O resultado a seguir é crucial para provar que a função definida acima é uma imersão

Proposição 1.2.14. Seja $K$ um subconjunto compacto de $\Omega$ e $\varphi \in \mathcal{A}_{0}(n)$. Então existe alguma vizinhança $U$ de $K$ e algum $\eta>0$ tal que

$$
\left(\theta\left(\varphi_{\varepsilon}\right) w\right) * \varphi_{\varepsilon}(x)=w * \varphi_{\varepsilon}(x)
$$

para todo $x \in U$ e para todo $0<\varepsilon<\eta$.

Teorema 1.2.15. A aplicação

$$
\begin{gathered}
\iota: \mathcal{D}^{\prime}(\Omega) \rightarrow \mathcal{G}_{f}(\Omega) \\
w \rightarrow\left[\left(\left.(\theta(\varphi) w * \varphi)\right|_{\Omega}\right)_{\varphi \in \mathcal{A}_{0}(n)}\right]
\end{gathered}
$$

é uma imersão linear que comuta com as derivadas parciais. Temos também que

(i) $\left.\iota\right|_{\mathcal{E}^{\prime}(\Omega)}=\iota_{0}$.

(ii) $\left.\iota\right|_{C^{\infty}(\Omega)}=\sigma$.

Demonstração. Vide [Kun96].

Proposição 1.2.16. $\hat{\imath}: \mathcal{D}^{\prime} \rightarrow \mathcal{G}_{f}$ é um morfismo de feixe(na categoria de espaço vetorial complexo), ou seja, para subconjuntos abertos $\Omega_{2} \subseteq \Omega_{1} \subseteq \mathbb{R}^{n}$ e $w \in \mathcal{D}^{\prime}\left(\Omega_{1}\right)$ temos que

$$
\left.\iota_{\Omega_{1}}(w)\right|_{\Omega_{2}}=\iota_{\Omega_{2}}\left(\left.w\right|_{\Omega_{2}}\right) .
$$

Demonstração. A prova é feita em [Kun96].

Finalmente podemos enunciar o

Teorema 1.2.17. Existe um único morfismo de feixe (de espaço vetoriais complexos), $\hat{\imath}$ : $\mathcal{D}^{\prime} \rightarrow \mathcal{G}_{f}$ que estende o mergulho canônico $\hat{\iota}_{0}: \mathcal{E}^{\prime} \rightarrow \mathcal{G}_{f}$ (ou seja, $\left.\iota_{\Omega}\right|_{\mathcal{E}^{\prime}(\Omega)}=\iota_{0 \Omega}$ para cada $\Omega \subseteq \mathbb{R}^{n}$ aberto). O $\hat{\iota}$ comuta com as derivadas parciais e sua restrição para $C^{\infty}$ é um morfismo de feixe de álgebras. 
O suporte de um elemento $U \in \mathcal{G}_{f}(\Omega)$ é definido como o complemento do maior conjunto aberto sobre o qual $U$ é nula. Temos:

Proposição 1.2.18. Para qualquer $w \in \mathcal{E}^{\prime}(\Omega)$,

$$
\operatorname{supp}(w)=\operatorname{supp}(\iota(w)) .
$$

Finalizamos esta seção com um resultado que relaciona a álgebra simplificada com a álgebra full.

Proposição 1.2.19. A aplicação

$$
\begin{gathered}
\zeta: \mathcal{G}(\Omega) \rightarrow \mathcal{G}_{f}(\Omega) \\
U \rightarrow\left[\left(u_{l(\varphi)}\right)_{\varphi}\right]
\end{gathered}
$$

é um monomorfismo de álgebras diferenciais.

\subsubsection{Pontos Generalizados}

Nesta seção estudaremos as funções generalizadas constantes, ou seja, os elementos de $\mathcal{G}(\Omega)$ que não dependem da variável $x$. isto nos leva à seguinte definição.

Definição 1.2.20. Para qualquer espaço normado suave E definamos,

$$
\begin{gathered}
\mathcal{E}_{f}(\mathbb{K} ; \mathbb{E}):=\left\{u: \mathcal{A}_{0}(E ; \mathbb{K}) \rightarrow \mathbb{K}: \exists p \in \mathbb{N}_{0}, \forall \varphi \in \mathcal{A}_{p}(E ; \mathbb{K}), \exists c>0 \exists \eta>0,\left|u\left(\varphi_{\varepsilon}\right)\right| \leq c \varepsilon^{-p}(0<\right. \\
\qquad<\eta)\} \\
\mathcal{N}_{f}(\mathbb{K} ; \mathbb{E}):=\left\{u: \mathcal{A}_{0}(E ; \mathbb{K}) \rightarrow \mathbb{K}: \exists p \in \mathbb{N}_{0}, \exists \gamma \in \Gamma, \forall q \geq p, \forall \varphi \in \mathcal{A}_{q}(E ; \mathbb{K}), \exists c>0, \exists \eta>\right. \\
\left.0,\left|u\left(\varphi_{\varepsilon}\right)\right| \leq c \varepsilon^{\gamma(q)-p}(0<\varepsilon<\eta)\right\} \\
\overline{\mathbb{K}}_{f}(E)=\mathcal{E}_{f}(\mathbb{K} ; \mathbb{E}) / \mathcal{N}_{f}(\mathbb{K} ; \mathbb{E})
\end{gathered}
$$

O próximo resultado relaciona o $\overline{\mathbb{K}}_{f}(E)$ quando os espaços normados suaves são diferentes e, consequentemente, mostra que eles são todos isomorfos, ou seja, independe do espaço $E$.

Proposição 1.2.21. Se E e F são espaços normados suave então $\overline{\mathbb{K}}_{f}(E)$ é canonicamente isomorfo com $\overline{\mathbb{K}}_{f}(F)$.

Demonstração. O isomorfismo é dado por $u \rightarrow u \circ I_{F}^{E}$ consulte [AB91a].

Fazendo uso dessa identificação poderemos omitir o espaço $E$, e simplesmente escreve$\operatorname{mos} \overline{\mathbb{K}}_{f}$ invés de $\overline{\mathbb{K}}_{f}(E)$. Os elementos de $\overline{\mathbb{K}}_{f}$ são chamados de números generalizados de Colombeau. Este anel tem divisores de zero e logo não é um corpo.

Vamos definir diametro do suporte de $\varphi$ por $\operatorname{diam}(\operatorname{supp}(\varphi))=\iota(\varphi)$, e veja o seguinte exemplo.

Exemplo 1.2.22. Definimos

$$
u(\varphi)= \begin{cases}0, & \text { se } l(\varphi)=\frac{1}{n}(n \in \mathbb{N}) \\ 1 & \text { se caso contrário }\end{cases}
$$

Então $0 \neq u \in \overline{\mathbb{K}}_{f}$ é um divisor de zero. 
Existe um monomorfismo canônico de $\mathbb{K}$ em $\overline{\mathbb{K}}_{f}: k \rightarrow\left[(k)_{\varphi}\right]$. Seja um aberto $\Omega \subset$ $\mathbb{R}^{n}$ vamos associar a $\Omega$ um subconjunto $\widetilde{\Omega}_{c} \subset \overline{\mathbb{K}}_{f}^{n}$. Os elementos de $\widetilde{\Omega}_{c}$ são chamados de pontos generalizados de $\Omega$. É o conjunto $\widetilde{\Omega}_{c}$ que vai permitir que distribuições sejam vistas como funções no sentido clássico. Começamos identificando os próprios elementos de $\Omega$ como pontos generalizados.

Definição 1.2.23. Seja $f \in \mathcal{G}_{f}(\Omega)$ e $x \in \Omega$. O valor de $f$ em $x$ é o elemento de $\overline{\mathbb{K}}_{f}$ definido $\operatorname{por}\left(f_{\varphi}(x)\right)_{\varphi}$.

O estudo das propriedades de $\overline{\mathbb{K}}_{f}$ foi feito na tese de doutorado de Ronaldo Garcia e publicado em conjunto com J. Aragona e S.O. Juriaans. Algumas das propriedades são que $\overline{\mathbb{K}}_{f}$ não tem elementos nilpotentes não nulos e que todo elemento é ou um divisor de zero ou é uma unidade, ou seja, um elemento invertível de $\overline{\mathbb{K}}_{f}$.

Definição 1.2.24. Seja $U \in \mathcal{G}_{f}(\Omega)$ e $M \subseteq \bar{M} \subset \subset \Omega$ um conjunto mensurável à Lebesgue. $A$ integral de $U$ sobre $M$ é o elemento

$$
\int_{M} U(x) d x=\left[\left(\int_{M} u_{\varphi}(x) d x\right)_{\varphi}\right]
$$

$d e \overline{\mathbb{K}}_{f}$.

O próximo teorema mostra uma propriedade interessante da noção de integração definida acima.

Teorema 1.2.25. Se $\omega \in \mathcal{D}^{\prime}(\Omega)$ e $\psi \in \mathcal{D}(\Omega)$ então

$$
\int_{\Omega} \iota(\omega)(x) \iota(\psi)(x) d x=<\omega, \psi>\text { em } \overline{\mathbb{K}}_{f} .
$$

Demonstração. Para a prova consulte [Kun96].

Isto sugere as seguintes definições.

Definição 1.2.26. Um elemento $U$ de $\mathcal{G}_{f}(\Omega)$ é chamado associado a 0 (denotada por $U \approx 0$ ) se para um representante $\left(u_{\varphi}\right)_{\varphi}$ (portanto para todos) a seguinte condição acontece existe $p \in \mathbb{N}$, para cada $\psi \in \mathcal{D}^{\prime}(\Omega)$.

$$
\lim _{\varepsilon \rightarrow 0} \int_{M} u\left(\varphi_{\varepsilon}, x\right) \psi(x) d x=0, \forall \varphi \in \mathcal{A}_{p}(n) .
$$

Definição 1.2.27. Seja $z \in \overline{\mathbb{K}}_{f}$ ele é associado a $0(z \approx 0)$ se para um representante $\left(z_{\varphi}\right)_{\varphi}$ (portanto para todos representantes) de $z$ temos: existe $p>0$ com $z\left(\varphi_{\varepsilon}\right) \rightarrow 0(\varepsilon \rightarrow 0)$ para todo $\varphi \in \mathcal{A}_{p}(n)$, então se $z_{1} \approx z_{2}$ significa $z_{1}-z_{2} \approx 0$. Se existe algum $a \in \mathbb{K}$ com $z \approx$ a então a é chamado número associado ou sombra de $z$.

As definições acima definem relações de equivalência sobre as respectivas álgebras. Chamamos esta relação de equivalência de associação.

Teorema 1.2.28. Temos as seguintes propriedades

1. Se $f \in C^{\infty}(\Omega)$ e $\omega \in \mathcal{D}^{\prime}(\Omega)$ então $\iota(f) \iota(\omega) \approx \iota(f \omega)$

2. Se $f, g \in C(\Omega)$ então $\iota(f) \iota(g) \approx \iota(f g)$.

3. Se $f \in C(\Omega)$ e $x_{0} \in \Omega$ então $\iota(f)\left(x_{0}\right) \approx f\left(x_{0}\right)$.

4. Seja $K \subset \subset \Omega$. Se $f \in C^{\infty}$ então $\int_{K} \iota(f)(x) d x=\int f(x) d x$ em $\overline{\mathbb{K}}_{f}$. Se $f \in L_{\text {loc }}^{1}(\Omega)$ ent $\tilde{a} o \int_{K} \iota(f)(x) d x \approx \int f(x) d x$. 
5. Para qualquer $f \in C\left(\mathbb{R}^{m+n}\right)$ temos que $\left.\iota(f)\right|_{\mathbb{R}^{m}} \approx \iota\left(\left.f\right|_{\mathbb{R}^{m}}\right)$.

6. Seja $g_{1}: \Omega_{1} \rightarrow \Omega_{2}$ uma função suave e $f \in C\left(\Omega_{2}\right)$. Então $\iota\left(f \circ g_{1}\right) \approx \iota(f) \circ g_{1}$.

7. Seja $g: \Omega_{1} \rightarrow \Omega_{2}$ um difeomorfismo e $\omega \in \mathcal{D}^{\prime}\left(\Omega_{2}\right)$. Então $\iota\left(g^{*}(\omega)\right) \approx \iota(\omega) \circ g$.

Com relação ao problema de multiplicação de distribuição temos o seguinte resultado.

Proposição 1.2.29. Se o produto $[u \cdot v]$ de $u, v \in \mathcal{D}^{\prime}\left(\mathbb{R}^{n}\right)$ existir então $\iota(u) \iota(v) \approx \iota([u \cdot v])$.

\subsubsection{Alguns Resultados na Álgebra $\mathcal{G}_{f}(\Omega)$}

Nesta seção vamos relembrar alguns resultados de [Nig13] e [Gar06]. Estamos interessado em estudar as propriedades dos conjuntos internos de [OV08] (que enunciaremos em breve), na álgebra plena de Colombeau. Começamos introduzindo a noção de pontos generalizados. Seja o conjunto de todos os pares $(\varphi, x) \in \mathcal{A}_{0}\left(\mathbb{R}^{n}\right) \times \Omega$ satisfazendo $\operatorname{supp}(\varphi)+x \subset \Omega$ com essa construção vejamos o seguinte, o leitor interessado pode consultar [Nig13] e [AB91b].

Definição 1.2.30. Dado $\Omega \subset \mathbb{R}^{n}$, definamos

$$
\begin{gathered}
\Omega_{M}(n):=\left\{X: \mathcal{A}_{0}\left(\mathbb{R}^{n}\right) \rightarrow \Omega \mid \forall \varphi \in \mathcal{A}_{0}\left(\mathbb{R}^{n}\right), \exists \varepsilon_{0}>0, \forall \varepsilon<\varepsilon_{0}:\left(\varphi_{\varepsilon}, X\left(\varphi_{\varepsilon}\right)\right) \in\right. \\
\left.U(\Omega), \quad \text { } \exists p \in \mathbb{N}, \forall \varphi \in \mathcal{A}_{p}\left(\mathbb{R}^{n}\right) ;\left|X\left(\varphi_{\varepsilon}\right)\right|=O\left(\varepsilon^{-p}\right)\right\} . \\
\Omega_{N}(n):=\left\{X \in \Omega_{M}(n)\left|\forall m \in \mathbb{N}, \exists q \in \mathbb{N}, \forall \varphi \in \mathcal{A}_{q}\left(\mathbb{R}^{n}\right) ;\right| X\left(\varphi_{\varepsilon}\right) \mid=O\left(\varepsilon^{q}\right)\right\} . \\
\widetilde{\Omega}(n):=\Omega_{M}(n) / \Omega_{N}(n) \\
\tilde{\Omega}_{c}(n):=\{\tilde{X} \in \tilde{\Omega}(n) \mid \text { para um representante } X \text { de } \tilde{X}, \exists K \subset \subset \Omega, \exists p \in \mathbb{N}, \forall \varphi \in \\
\left.\mathcal{A}_{p}\left(\mathbb{R}^{n}\right), \forall \varepsilon \in I_{\eta} ; X\left(\varphi_{\varepsilon}\right) \in K\right\}
\end{gathered}
$$

Os elementos do último conjunto são chamados pontos compactamente suportados ou pontos generalizados.

Proposição 1.2.31. Sejam $x \in \widetilde{\Omega}_{c}(n)$ e seja $f \in \mathcal{G}_{f}(\Omega)$. Então o elemento $f(x)=$ $\left[\left(f_{\varphi}\left(x_{\varphi}\right)\right)_{\varphi}\right] \in \overline{\mathbb{K}}_{f}$ está bem definida.

O elemento $f(x)$ do resultado anterior é chamado o valor da $f$ em $x$. Isto estende a noção de valor num ponto $x \in \Omega$ visto anteriormente.

Lema 1.2.32. Dado $\varphi \in \mathcal{A}_{q}\left(\mathbb{R}^{n}\right), \varepsilon_{q, k} \in(0, \infty)$ e $x_{0}, x_{q, k}$ em qualquer conjunto $A$ para todo $q, k \in \mathbb{N}$, existe uma aplicação $X: \mathcal{A}_{0}\left(\mathbb{R}^{n}\right) \rightarrow A$ e sequências estritamente crescentes $\left(q_{l}\right)_{l \in \mathbb{N}}$ $e\left(a_{l}\right)_{l \in \mathbb{N}}$ de números naturais tais que $X\left(\varphi_{q_{l}, \varepsilon_{q, k}}\right)=x_{q_{l}, k}, \forall l, k \in \mathbb{N}$, e $X(\varphi)=x_{0}$ para todo $\varphi$ que não esta em alguma $\varphi_{q_{l}, \varepsilon_{q, k}}$, e $\varphi_{q_{l}} \in \mathcal{A}_{a_{l}}\left(\mathbb{R}^{n}\right) / \mathcal{A}_{a_{l+1}}\left(\mathbb{R}^{n}\right)$.

O resultado principal desta seção permite que funções generalizadas sejam vistas como funções clássicas.

Teorema 1.2.33. Seja $f \in \mathcal{G}_{f}(\Omega)$. Então $f=0$ se e somente se, $f(x)=0, \forall x \in \tilde{\Omega}_{c}(n)$.

Podemos também provar que na álgebra diferencial $\mathcal{G}_{f}(\Omega)$ os elementos constantes são exatamente aquelas cujas derivadas são zero, ou seja, os elementos de $\overline{\mathbb{K}}_{f}$.

Lema 1.2.34. Seja $\Omega \subseteq \mathbb{R}^{n}$ conexo e $K \subset \subset \Omega$. Então existe um conjunto $M \subset \subset \Omega$ contendo $K$ e um número real $L>0$ tal que qualquer dois pontos em $K$ pode ser ligados por uma curva $\gamma:[0,1] \rightarrow \Omega$ com imagem em $M$ tendo comprimento $\int_{0}^{1}\left|\gamma^{\prime}(t)\right| d t \geq L$. 
Definição 1.2.35. Para $r, s \in \overline{\mathbb{K}}_{f}$ escrevemos $r \leq s$ se existir representantes destes elementos tal que $r(\varphi) \leq s(\varphi)$ para todo $\varphi \in \mathcal{A}_{0}(\Omega)$.

Proposição 1.2.36. O anel $(\tilde{\mathbb{R}}(n), \leq)$ é parcialmente ordenado. que

Um elemento $r \in \overline{\mathbb{K}}_{f}$ é estritamente diferente de zero, se existir um representante tal

$$
\exists q \in \mathbb{N}, \varphi \in \mathcal{A}_{q}\left(\mathbb{R}^{n}\right), \exists C>0, \eta>0 ; \forall \varepsilon<\eta:\left|r\left(\varphi_{\varepsilon}\right)\right|>C \varepsilon^{q} .
$$

Isto é usado para caracterizar elementos invertíveis.

Proposição 1.2.37. Um elemento de $r \in \overline{\mathbb{K}}_{f}$ é invertível se, e somente se, $r$ é estritamente diferente de zero.

Teorema 1.2.38. Seja $f \in \mathcal{G}_{f}(\Omega)$. Então $f$ é invertível se, e somente se, $f(x)$ é invertível $\forall x \in \widetilde{\Omega}_{c}$.

\subsection{Topologias Naturais Sobre as Álgebras de Colom- beau em $\mathcal{G}_{f}(\Omega)$}

\subsubsection{A topologia Sharp sobre $\mathcal{G}_{f}(\Omega)$}

Seja $\Omega \subset \mathbb{R}^{n}$ e $K \subset \subset \Omega$ um subconjunto relativamente compacto de $\Omega$. Dada $f: \Omega \rightarrow \mathbb{K}$ definimos

$$
\|f\|_{K}:=\sup _{x \in K}\|f(x)\|
$$

No caso em que $\left\{\Omega_{m}\right\}_{m \in \mathbb{N}}$ é uma exaustão de $\Omega$, escrevemos $\|f\|_{m}$ ao invés de $\|f\|_{\Omega_{m}}$ iremos ver alguns resultados obtidos por Aragona-Fernandez-Juriaans em [AFJ $\left.{ }^{+} 09\right]$.

Proposição 1.3.1. Sejam

(a) $u \in \mathcal{E}_{M, f}(\Omega)$ se, e somente se, $\forall(m, p) \in \mathbb{N}^{2}, \exists N \in \mathbb{N}, \exists a \in \mathbb{R}$ tal que $\forall \varphi \in \mathcal{A}_{N}(\mathbb{K})$ temos $\left\|\partial^{\beta} u\left(\varphi_{\varepsilon}, \cdot\right)\right\|_{m}=o\left(\varepsilon^{a}\right)$, se $\varepsilon \rightarrow 0, \forall|\beta| \leq p$;

(b) $u \in \mathcal{N}_{f}(\Omega)$ se, e somente se, $\forall(m, p) \in \mathbb{N}^{2}, \forall a \in \mathbb{R}, \exists N \in \mathbb{N}$, tal que $\forall \varphi \in \mathcal{A}_{N}(\mathbb{K})$ temos $\left\|\partial^{\beta} u\left(\varphi_{\varepsilon}, \cdot\right)\right\|_{m}=o\left(\varepsilon^{a}\right)$, se $\varepsilon \rightarrow 0, \forall|\beta| \leq p$.

Dado $r \in \mathbb{R}$ lembramos que

$$
\alpha_{r}^{\bullet}(\varphi)=i(\varphi)^{r}, i(\varphi)=l(\varphi)
$$

Observamos que temos que $\alpha_{r}^{\bullet}\left(\varphi_{\varepsilon}\right)=\varepsilon^{r} i(\varphi)$. Por consequência, temos:

Corolário 1.3.2. (a) Se $u \in \mathcal{E}_{M, f}(\Omega)$, então para todo $(m, p) \in \mathbb{N}^{2}$ existem $N \in \mathbb{N}, r \in \mathbb{R}$ tais que $\forall \varphi \in \mathcal{A}_{N}(\mathbb{K})$ existe $\eta=\eta(\varphi) \in I$ tal que

$$
\left\|\partial^{\beta} u\left(\varphi_{\varepsilon}, \cdot\right)\right\|_{m} \leq \alpha_{r}^{\bullet}\left(\varphi_{\varepsilon}\right), \forall \varepsilon \in I_{\eta}, \forall|\beta| \leq p
$$

(b) Se $u \in \mathcal{N}_{f}(\Omega)$, então para todo $(m, p) \in \mathbb{N}^{2}$, para todo $r \in \mathbb{R}$ existe $N \in \mathbb{N}$, tal que $\forall \varphi \in \mathcal{A}_{N}(\mathbb{K})$ existe $\eta=\eta(\varphi) \in I$ com a propriedade 


$$
\left\|\partial^{\beta} u\left(\varphi_{\varepsilon}, \cdot\right)\right\|_{m} \leq \alpha_{r}^{\bullet}\left(\varphi_{\varepsilon}\right), \forall \varepsilon \in I_{\eta}, \forall|\beta| \leq p .
$$

Demonstração. Consulte [AFJ05].

Lema 1.3.3. Para todo $u \in \mathcal{E}_{M, f}(\Omega), \beta \in \mathbb{N}^{n}$ e $m \in \mathbb{N}$, temos:

(a) A função $u_{m}^{\beta}: \mathcal{A}_{0}(\mathbb{K}) \rightarrow \mathbb{R}_{+}$tal que $u_{m}^{\beta}=\left\|\partial^{\beta} u(\varphi, \cdot)\right\|_{m}$ é moderada, ou seja, $u_{m}^{\beta} \in$ $\mathcal{E}_{M, f}(\mathbb{R})$ e $\left[\left(u_{m}^{\beta}\right)\right] \in \overline{\mathbb{R}}_{f,+} ;$

(b) Se $v \in \mathcal{E}_{M, f}(\Omega)$ e $u-v \in \mathcal{N}_{f}(\Omega)$, então $u_{m}^{\beta}-v_{m}^{\beta} \in \mathcal{N}_{f}(\mathbb{R})$ e portanto $\left[\left(u_{m}^{\beta}\right)\right]=\left[\left(v_{m}^{\beta}\right)\right]$.

Estamos agora em condições para introduzir a topologia sharp sobre $\mathcal{G}_{f}(\Omega)$.

Definição 1.3.4. Seja $\left\{\Omega_{m}\right\}_{m \in \mathbb{N}}$ uma exaustão aberta de $\Omega$. Dado $u \in \mathcal{E}_{M, f}(\Omega)$ e $(m, p) \in$ $\mathbb{N}^{2}$, definimos:

$$
A_{m p}(u):=\left\{r \in \mathbb{R}\left|\forall \beta \in \mathbb{N}^{n},\right| \beta \mid \leq p \text { tem-se } \dot{\alpha}_{-r} u_{m}^{\beta} \approx 0\right\}
$$

e chamamos a valuação de u ao supremo de $A_{m p}(u)$, ou seja,

$$
V_{m p}(u)=\sup \left(A_{m p}(u)\right)
$$

Lema 1.3.5. Tem-se que $r \in A_{m p}(u)$ se, se e somente se, para todo $\beta \in \mathbb{N}^{n}$, com $|\beta| \leq p$, existe $N \in \mathbb{N}$ tal que

$$
\lim _{\varepsilon \rightarrow 0} \varepsilon^{-r} u_{m}^{\beta}\left(\varphi_{\varepsilon}\right)=0, \forall \varphi \in \mathcal{A}_{N}(\mathbb{K})
$$

Proposição 1.3.6. Se $a \in A_{m p}(u)$ e $b<a$, então $b \in A_{m p}(u)$

Listamos agora algumas propriedades da valuação acima.

Proposição 1.3.7. Para todos $u, v \in \mathcal{E}_{M, f}(\Omega)$ e $(m, p) \in \mathbb{N}^{2}$ e $\lambda \in \mathbb{K}$, temos:

(a) $V_{m p}(\lambda u)=V_{m p}(u)$, se $\lambda \neq 0$;

(b) $V_{m p}(u v) \geq V_{m p}(u)+V_{m p}(v)$;

(c) $V_{m p}\left(\alpha_{r}^{\bullet} u\right)=r+V_{m p}(u), \forall r \in \mathbb{R}$

(d) $V_{m p}(u+v) \geq \inf \left\{V_{m p}(u), V_{m p}(v)\right\}$;

(e) $u \in \mathcal{N}_{f}(\Omega) \Leftrightarrow A_{m p}(u)=\mathbb{R}, \forall(m, p) \in \mathbb{N}^{2} \Leftrightarrow V_{m p}(u)=+\infty, \forall(m, p) \in \mathbb{N}^{2}$.

(f) $V_{m p}$ é constante sobre classe de equivalência módulo $\mathcal{N}_{f}(\Omega)$.

Proposição 1.3.8. A função $D_{m p}(f, g): \mathcal{G}_{f}(\Omega) \times \mathcal{G}_{f}(\Omega) \rightarrow \mathbb{R}_{+}$definida por $D_{m p}(f, g)=$ $e^{-V_{m p}(\hat{f}-\hat{g})}$ onde $\hat{f}$ e $\hat{g}$ são representantes quaisquer de $f$ e $g$, respectivamente, é uma pseudoultramétrica sobre $\mathcal{G}_{f}(\Omega)$.

A estrutura uniforme determinada pela família $\left\{D_{m p}\right\}_{(m, p) \in \mathbb{N}^{2}}$ é chamada a estrutura uniforme cortante(sharp) sobre $\mathcal{G}_{f}(\Omega)$, e a denotaremos por $\tau_{\Omega, f}$.

Proposição 1.3.9. Para todo $(m, p) \in \mathbb{N}^{2}$ a pseudo-ultramétrica $D_{m p}$ é invariante por translação. Mais ainda, para todo $f \in \mathcal{G}_{f}(\Omega)$ e $\alpha \in \mathbb{N}^{n}$ as seguintes afirmações se verificam:

(a) $f=0$ se, e somente se, para algum(ou equivalentemente para todo) representante de $\hat{f}$ de $f$ temos que $V_{m p}(\hat{f})=+\infty$, ou seja, $D_{m p}(f, 0)=0$ para todo $(m, p) \in \mathbb{N}^{2}$.

(b) $D_{m p}\left(\partial^{\alpha} f, 0\right) \leq D_{m(p+\alpha)}(f, 0)$ para todo $(m, p) \in \mathbb{N}^{2}$.

Mais detalhes sobre esta topologia o leitor pode encontrar em [AFJ05]. 


\subsubsection{Propriedades de $\overline{\mathbb{K}}_{f}$}

Lembramos que o anel de números generalizados de Colombeau é definido por

$$
\overline{\mathbb{K}}_{f}=\mathcal{E}_{M, f}(\mathbb{K}) / \mathcal{N}_{f}(\mathbb{K})
$$

e que $\mathbb{K}$ está contido em $\overline{\mathbb{K}}_{f}$ via a imersão constante,

$$
\begin{gathered}
\mathbb{K} \hookrightarrow \overline{\mathbb{K}}_{f} \\
k \mapsto\left[(k)_{\varphi}\right]
\end{gathered}
$$

onde $c(\varphi)=c, \forall \varphi \in \mathcal{A}_{0}(\mathbb{K})$. Podemos ver a seguinte definição, já feita anteriormente, mas aqui apresentamos com mais clareza sobre o tema.

Definição 1.3.10. Um elemento $v \in \overline{\mathbb{K}}_{f}$ é associado ao zero e denotaremos por $v \approx 0$ se para um(e portanto para todos) representantes $v(\varphi)_{\varphi}$ de $v$, existe $p \in \mathbb{N}^{*}$ tal que :

$$
\lim _{\varepsilon \rightarrow 0} v\left(\varphi_{\varepsilon}\right)=0, \forall \varphi \in \mathcal{A}_{p}(\mathbb{K}) .
$$

Dizemos que $v_{1} \approx v_{2}$ se $\left(v_{1}-v_{2}\right) \approx 0$. Se existe $a \in \mathbb{K}$ tal que $v \approx a$ então $a$ é chamado de número associado, ou sombra de $v$.

Sejam $\Omega \subset \mathbb{R}^{n}$ um aberto, e $\varphi \in D(\Omega), \iota(\varphi)=\operatorname{diam}(\operatorname{supp} \varphi)$. Notemos que $\iota\left(\varphi_{\varepsilon}\right)=\varepsilon \iota(\varphi)$, $\forall \varepsilon \in(0,1]$. Como já foi feito anteriormente, para cada $r \in \mathbb{R}$ definimos:

$$
\alpha_{r}^{\bullet}: \mathcal{A}_{0}(\mathbb{K}) \rightarrow \mathbb{R} \text { por } \alpha_{r}^{\bullet}(\varphi):=(\iota(\varphi))^{r} .
$$

e notamos que $\alpha_{r}^{\bullet}\left(\varphi_{\varepsilon}\right):=\varepsilon^{r}\left(\alpha_{r}^{\bullet}(\varphi)\right)$. Aragona- Juriaans em [AJ01] definiram, para $r \in \mathbb{R}$, o elemento

$$
\alpha_{r}: \mathcal{A}_{0}(\mathbb{K}) \rightarrow \mathbb{R} \text { por } \alpha_{r}(\varepsilon):=\varepsilon^{r},
$$

e com isto temos que $\alpha_{r}^{\bullet}\left(\varphi_{\varepsilon}\right):=\alpha_{r}(\varepsilon)\left(\alpha_{r}^{\bullet}(\varphi)\right)$.

Definição 1.3.11. Para $x \in \overline{\mathbb{K}}_{f}$ definamos,

$$
A(x):=\left\{r \in \mathbb{R} \mid \alpha_{-r}^{\bullet} x \approx 0\right\}
$$

e a valoração $V(x)$ de $x$ é $V(x):=\sup (A(x))$.

Lema 1.3.12. Seja $x \in \overline{\mathbb{K}}_{f}$. Então $r \in A(x)$ se, e somente se, existe $p \in \mathbb{N}$ tal que,

$$
\lim _{\varepsilon \rightarrow 0} \varepsilon^{-r} x\left(\varphi_{\varepsilon}\right)=0, \forall \varphi \in \mathcal{A}_{p}(\mathbb{K}) .
$$

Listamos as propriedade de $V(x)$ abaixo.

Proposição 1.3.13. Para $x, y \in \mathcal{E}_{M, f}(\mathbb{K})$ e $\lambda \in \mathbb{K}$, temos:

(a) $V(\lambda x)=V(x)$ se $\lambda \neq 0$

(b) $V(x y) \geq V(x)+V(y)$

(c) $V\left(\alpha_{r}^{\bullet} x\right)=r+V(x)$

(d) $V(x+y) \geq \inf \{V(x), V(y)\}$

(e) $V(x)=\infty \Leftrightarrow x \in \mathcal{N}_{f}(\mathbb{K})$ 
(f) $V$ é constante sobre cada classe de equivalência módulo $\mathcal{N}_{f}(\mathbb{K})$, isto é,

$$
V(x)=V(x+y), \forall x \in \mathcal{E}_{M, f}(\mathbb{K}), \forall y \in \mathcal{N}_{f}(\mathbb{K}) .
$$

Definimos

$$
\begin{aligned}
& D: \overline{\mathbb{K}}_{f} \times \overline{\mathbb{K}}_{f} \rightarrow \mathbb{R}_{+} \\
& D(x, y):=e^{-V(x-y)}
\end{aligned}
$$

Corolário 1.3.14. A função D é uma ultra-métrica sobre $\overline{\mathbb{K}}_{f}$ que é invariante por translação.

$D$ determina uma ultramétrica sobre $\overline{\mathbb{K}}_{f}$, chamada de topologia cortante e a denotamos por $\tau_{s f}$.

Sejam $x \in \overline{\mathbb{K}}_{f}$ e $r \in \mathbb{R}_{+}^{*}$, e denotaremos por $B_{r}(x)\left(\right.$ resp. $B_{r}^{\prime}(x)$ e $\left.S_{r}(x)\right)$ a $D$-bola aberta (resp. D-bola fechada e D-esfera) com centro em $x$ e raio $r$.

Quando $x=0$ simplesmente escreveremos $B_{r}\left(\right.$ resp. $B_{r}^{\prime}$ e $\left.S_{r}\right)$. Para simplificarmos definimos:

$$
\|x\|:=D(x, 0), \forall x \in \overline{\mathbb{K}}_{f}
$$

como a distancia entre dois elementos $x, y \in \overline{\mathbb{K}}_{f}$ e dado por $D(x, y):=\|x-y\|$.

Algumas propriedades da ultramétrica são listadas a seguir.

Corolário 1.3.15. Para $x, y \in \overline{\mathbb{K}}_{f}, r \in \mathbb{R}$ e $s \in \mathbb{R}_{+}^{*}$ e $a, b \in \mathbb{K}$, temos:

(a) $\|x+y\| \leq \max \{\|x\|,\|y\|\}$ e $\|x y\| \leq\|x\|\|y\|$

(b) $\|x\|=0 \Leftrightarrow x=0$

(c) $\|a x\|=\|x\|$, se $a \neq 0$

(d) $\left\|\alpha_{r}^{\bullet} x\right\|=\left\|\alpha_{r}^{\bullet}\right\|\|x\|$

(e) $\|a\|=1$, se $a \neq 0$

(f) $\|a-b\|=1-\delta_{a b}$ ( $\delta$ de Kronecker).

Proposição 1.3.16. $\left(\overline{\mathbb{K}}_{f}, \tau_{s f}\right)$ é um anel topológico completo.

Proposição 1.3.17. Temos as propriedades.

(a) $\left.\overline{\mathbb{K}}_{f,} \tau_{s f}\right)$ não é um $\mathbb{K}$ - espaço vetorial topológico.

(b) $\left(\overline{\mathbb{K}}_{f,} \tau_{s f}\right)$ não é separável.

(c) $\left(\overline{\mathbb{K}}_{f}, \tau_{s f}\right)$ não é localmente compacto.

Proposição 1.3.18. Sejam $\left\{a_{n}\right\}_{n \in \mathbb{N}} \subset \mathbb{K}$ uma sequência qualquer e $0 \neq x \in B_{1}$, ou seja, $\|x\|<1$.Então a série

$$
\sum_{n \geq 0} a_{n} x^{n}
$$

converge em $\overline{\mathbb{K}}_{f}$. Em particular, se $a_{n}=1, \forall n \in \mathbb{N}$, então $\sum_{n \geq 0} x^{n}$ converge e temos $(1-x) \sum_{n \geq 0} x^{n}=1$. Neste caso $(1-x) \in \operatorname{Inv}\left(\overline{\mathbb{K}}_{f}\right)$.

Como consequência desse resultado temos o seguinte.

Corolário 1.3.19. Conjunto dos invertíveis de $\overline{\mathbb{K}}_{f}$ que denotamos por Inv $\left(\overline{\mathbb{K}}_{f}\right)$ é um subconjunto aberto de $\overline{\mathbb{K}}_{f}$.

O próximo teorema mostra que os ideias maximais de $\overline{\mathbb{K}}_{f}$ são fechados.

Teorema 1.3.20. Seja $\mathcal{J}$ um ideal próprio de $\overline{\mathbb{K}}_{f}$. Então para cada $x \in \mathcal{J}$, temos $D(1, x) \geq$ 1 e $D(1,0)=1$. Portanto, todo ideal maximal de $\overline{\mathbb{K}}_{f}$ é fechado. 


\subsubsection{Cálculo Diferencial sobre $\overline{\mathbb{K}}^{n}$}

Vamos considerar $\overline{\mathbb{K}}$ com a topologia cortante e $\overline{\mathbb{K}}^{n}$ com a topologia produto e a norma do máximo. Vamos introduzir um calculo diferencial em $\overline{\mathbb{K}}$ apresentando tudo na álgebra simplificada mas que também vale para a álgebra full.

Se $r \in \mathbb{R}_{+}$e $x_{0} \in \overline{\mathbb{K}}^{n}$ então $B_{r}\left(x_{0}\right), B_{r}^{\prime}\left(x_{0}\right)$ e $S_{r}\left(x_{0}\right)$ representa os elementos $x \in \overline{\mathbb{K}}^{n}$ de modo que $\left\|x-x_{0}\right\|<r,\left\|x-x_{0}\right\| \leq r e\left\|x-x_{0}\right\|=r$ respectivamente.

Lema 1.3.21. Sejam $U \subset \overline{\mathbb{K}}$ um subconjunto aberto e $f: U \rightarrow \overline{\mathbb{K}}$ com $x_{0} \in U$. Então existe no máximo um elemento $z_{0} \in \overline{\mathbb{K}}$ tal que

$$
\lim _{x \rightarrow x_{0}} \frac{f(x)-f\left(x_{0}\right)-z_{0}\left(x-x_{0}\right)}{\alpha_{-l n\left\|x-x_{0}\right\|}}=0
$$

Demonstração. Veja os detalhes em [AFJ05].

Definição 1.3.22. Dado um conjunto aberto $U \subset \overline{\mathbb{K}}$, e $f: U \rightarrow \overline{\mathbb{K}}$ e $x_{0} \in U$ dizemos que $f$ é diferenciável em $x_{0}$ se existir $z_{0} \in \overline{\mathbb{K}}$ tal que

$$
\lim _{x \rightarrow x_{0}} \frac{f(x)-f\left(x_{0}\right)-z_{0}\left(x-x_{0}\right)}{\alpha-\log \left\|x-x_{0}\right\|}=0
$$

Neste caso, $f$ é dito diferenciável em $x_{0}$, e escrevemos $D(f)\left(x_{0}\right)=z_{0}$ e chamamos $z_{0}$ a derivada de $f$ em $x_{0}$. Dizemos que $f$ é diferenciável, se $f$ é diferenciável em cada ponto do dominio.

A função $f$ ser diferenciável em $x_{0}$ é equivalente à afirmação,

$$
\lim _{x \rightarrow x_{0}} \frac{\|T(x)\|}{\left\|x-x_{0}\right\|}=0
$$

onde $T(x):=f(x)-f\left(x_{0}\right)-D f\left(x_{0}\right)\left(x-x_{0}\right)$.

Como $\overline{\mathbb{K}}$ não é um corpo, somente podemos formar o quociente de dois dos seus elementos se o denominador for invertivel. A escolha de $\alpha_{-\log \left\|x-x_{0}\right\|}$ é natural pois $\left\|\alpha_{-l o g}\right\| x-x_{0}\|\|=$ $\left\|x-x_{0}\right\|$.

Se tivessemos definido a diferenciabilidade de $f$ em $x_{0}$ por $\lim _{x \rightarrow x_{0}} \frac{T(x)}{\left\|x-x_{0}\right\|}=0$ teríamos que a continuidade de $f$ em $x_{0}$ implicaria a diferenciabilidade em $x_{0}$. A nossa definição implica que $f(x)-f\left(x_{0}\right)=D(f)\left(x_{0}\right)\left(x-x_{0}\right)+E(x)$ com $\lim _{x \rightarrow x_{0}} \frac{E(x)}{\alpha_{-\log \left\|x-x_{0}\right\|}}=0$. Em particular, se a função for diferenciável em $x_{0}$ então $D(f)\left(x_{0}\right)=\lim _{n \rightarrow \infty} \frac{f\left(x+\alpha_{n}\right)-f\left(x_{0}\right)}{\alpha_{n}}$.

Lema 1.3.23. Sejam $U \subset \overline{\mathbb{K}}$ um subconjunto aberto, se $f: U \rightarrow \overline{\mathbb{K}}$ é diferenciável em $x_{0}$, então f é contínua neste ponto.

Demonstração. Consulte a prova em [AFJ05].

A noção de derivada definida acima tem as mesmas propriedades que a derivada clássica. Tem-se:

Proposição 1.3.24. Seja $U \subset \overline{\mathbb{K}}$ um subconjunto aberto e $f, g: U \rightarrow \overline{\mathbb{K}}$ funções diferenciáveis. Então:

(1) $f g$ é diferenciável e $D(f g)=g D(f)+f D(g)$;

(2) Se $f(U)$ está contido no dominio de $g$, então $D(g \circ f)=(D(g) \circ f) D(f)$;

(3) $D(f \pm g)=D(f) \pm D(g)$;

(4) Se $g(x)$ é invertivel para cada $x \in U$ temos que $D\left(\frac{f}{g}\right)=\frac{g D(f)-f D(g)}{g^{2}}$. 
Vamos agora estender as definições para $\overline{\mathbb{K}}^{n}$, temos:

Definição 1.3.25. Sejam $U \subset \overline{\mathbb{K}}^{n}$ um subconjunto aberto e $f: U \rightarrow \overline{\mathbb{K}}$ e $x=\left(x_{1}, \ldots, x_{n}\right), x=$ $\left(x_{01}, \ldots, x_{0 n}\right) \in U$. Seja $1 \leq i \leq n$ e suponhamos que exista um elemento $a_{i}$ de $\overline{\mathbb{K}}$ tal que

$$
\lim _{h \rightarrow 0} \frac{f\left(x_{01}, \ldots, x_{0 i}+h, \ldots, x_{0 n}\right)-f\left(x_{01}, \ldots, x_{0 i}, \ldots, x_{0 n}\right)-a_{i} h}{\alpha_{-l o g}\|h\|}=0
$$

Definimos $\frac{\partial f}{\partial x_{i}}\left(x_{0}\right):=a_{i}$, chamado a derivada parcial de $f$ com respeito a $x_{i}$ em $x_{0}$.

A função $f$ é dito diferenciável em $x_{0}$ se existir $a=\left(a_{1}, \ldots, a_{n}\right) \in \overline{\mathbb{K}}^{n}$ tal que

$$
\lim _{x \rightarrow x_{0}} \frac{f(x)-f\left(x_{0}\right)-\sum_{1 \leq i \leq n} a_{i}\left(x_{i}-x_{i 0}\right)}{\alpha_{-\log \left\|x-x_{0}\right\|}}=0
$$

$O$ gradiente de $f$ em $x_{0}$ é $\nabla f\left(x_{0}\right):=\left(\frac{\partial f}{\partial x_{1}}\left(x_{0}\right), \ldots, \frac{\partial f}{\partial x_{n}}\left(x_{0}\right)\right)$.

Se $U \subset \overline{\mathbb{R}}^{n}$ e $k \in \mathbb{N}$, então definimos o conjunto,

$$
\begin{gathered}
\mathcal{C}^{k}(U, \overline{\mathbb{K}}):=\left\{f: U \rightarrow \overline{\mathbb{K}} \mid \partial^{\alpha} f \in \mathcal{C}(U, \overline{\mathbb{K}}) \text { para todo } \alpha \in \mathbb{N}^{n} \text { tal que } 0 \leq|\alpha| \leq k\right\} \\
\mathcal{C}^{\infty}(U, \overline{\mathbb{K}}):=\cap_{k \in \mathbb{N}} \mathcal{C}^{k}(U, \overline{\mathbb{K}})
\end{gathered}
$$

Definição 1.3.26. Sejam $U \subset \overline{\mathbb{R}}^{n}$ um subconjunto aberto e $f: U \rightarrow \overline{\mathbb{R}}^{m}$. Escrevemos $f=\left(f_{1}, \ldots, f_{m}\right)$ onde cada $f_{i}: U \rightarrow \overline{\mathbb{R}}$. Vamos dizer que $f$ é diferenciável em $x_{0} \in U$, se cada $f_{i}$ for diferenciável em $x_{0}$

Observação 1.3.27. Temos que $f$ é diferenciável em $x_{0}$ se, e somente se, existe uma $\overline{\mathbb{R}}-$ linear aplicação $T: \overline{\mathbb{R}}^{n} \rightarrow \overline{\mathbb{R}}^{m}$ tal que,

$$
\lim _{x \rightarrow x_{0}} \frac{f(x)-f\left(x_{0}\right)-T\left(x-x_{0}\right)}{\alpha_{-} \log \left\|x-x_{0}\right\|}=0 .
$$

A aplicação T será denotada por $\operatorname{Df}\left(x_{0}\right)$.

\subsection{Análise Não Standard}

Em [Ver11] H. Vernaeve mostrou que a álgebra simplificada de Colombeau satisfaz os axiomas de análise não standard. Vamos relembrar alguns destes conceitos que mais tarde serão generalizadas para a álgebra full. Entre estes conceitos estão o princípio da saturação, o overspil e overflow.

Definição 1.4.1. Seja I um conjunto infinito. O conjunto $A \subseteq \mathcal{P}(I)$ é um filtro(proprio) sobre I se,

- $I \in A e \emptyset \notin A$

- Se $B_{1}, B_{2} \in A$, então $B_{1} \cap B_{2} \in A$

- Se $B \in A$ e $B \subseteq G \subseteq I$, então $G \subseteq A$

Um filtro é maximal se ele não está contido propriamente em qualquer outro filtro sobre I. 
Um filtro $\mathcal{F}$ sobre um conjunto I é livre se $\cap_{S \in \mathcal{F}} S=\emptyset$. Uma propriedade $P_{\varepsilon}$ dependendo de $\varepsilon \in I$ acontece quase sempre se, e somente se, $\left\{\varepsilon \in I: P_{\varepsilon}\right\}$ pertence a $\mathcal{F}$. Vamos considerar I um conjunto de indice infinito e fixamos um filtro livre sobre $I$. Em particular, para aplicações da teoria de funções generalizadas não linear, mantemos a escolha,

$I=\mathbb{N}$ com $\mathcal{F}=\{s \subseteq \mathbb{N} \mid \mathbb{N} / s$ é finito $\}$ ( $\mathcal{F}$ é então chamado filtro de Fréchert),ou $I=(0,1]$ com $\mathcal{F}=\{S \subseteq(0,1]: \exists \eta \in I=(0,1] e(0, \eta) \subseteq S\}$

Então a propriedade $P_{\varepsilon}$ dependendo de $\varepsilon \in \mathbb{N}($ resp. $\varepsilon \in(0,1])$ acontece quase sempre, denotada por q.s., se e somente se, $\varepsilon$ acontece para $\varepsilon$ suficientemente grande $\varepsilon \in \mathbb{N}$ (resp. para $\varepsilon \in(0,1]$ suficientemente pequeno). Por questão de generalidade, desenvolvemos a teoria para qualquer filtro livre $\mathcal{F}$ sobre qualquer conjunto de indice infinito.

Como em analise não standard (ou seja, o caso em que o filtro $\mathcal{F}$ é filtro maximal), definimos números reais generalizados como elementos de ${ }^{*} \mathbb{R}:=\mathbb{R}^{I} / \mathcal{F}$, famílias $\left(a_{\varepsilon}\right)_{\varepsilon \in I}$ de números reais modulo $\mathcal{F}$. Então, para classes equivalentes $\left[\left(a_{\varepsilon}\right)_{\varepsilon}\right],\left[\left(b_{\varepsilon}\right)_{\varepsilon}\right]$ temos,

$$
\left[\left(a_{\varepsilon}\right)_{\varepsilon}\right]=\left[\left(b_{\varepsilon}\right)_{\varepsilon}\right] \Longleftrightarrow a_{\varepsilon}=b_{\varepsilon} \text { q.s. }
$$

Além disso, definimos indutivamente o objeto chamado interno como segue:

1.Por definição, elementos de $* \mathbb{R}$ são objetos interno.

2. Seja $m \in \mathbb{N}$. Se $\left[\left(a_{1, \varepsilon}\right)_{\varepsilon}\right], \ldots,\left[\left(a_{n, \varepsilon}\right)_{\varepsilon}\right]$ são objetos internos, então

$$
\left[\left(\left(a_{1, \varepsilon}\right)_{\varepsilon}, \ldots,\left(a_{m, \varepsilon}\right)_{\varepsilon}\right)\right]:=\left(\left[\left(a_{1, \varepsilon}\right)_{\varepsilon}\right], \ldots,\left[\left(a_{m, \varepsilon}\right)_{\varepsilon}\right]\right)
$$

é um objeto interno.

3. Se $A_{\varepsilon}$ são conjuntos não vazios, (para cada $\left.\varepsilon \in I\right)$ tal que para cada escolha de $a_{\varepsilon} \in A_{\varepsilon}$, $\left[\left(a_{\varepsilon}\right)_{\varepsilon}\right]$ é um objeto interno, então,

$$
\left[\left(A_{\varepsilon}\right)_{\varepsilon}\right]:=\left\{\left[\left(a_{\varepsilon}\right)_{\varepsilon}\right]: a_{\varepsilon} \in A_{\varepsilon}\right\} \text { q.s. }
$$

é um objeto interno.

Lema 1.4.2. Seja $\left[\left(a_{\varepsilon}\right)_{\varepsilon}\right],\left[\left(a_{\varepsilon}\right)_{\varepsilon}\right]$ objetos internos. Então

1. $\left[\left(a_{\varepsilon}\right)_{\varepsilon}\right]=\left[\left(b_{\varepsilon}\right)_{\varepsilon}\right]$ se, somente se, $a_{\varepsilon}=b_{\varepsilon}$ q.s.

2. $\left[\left(a_{\varepsilon}\right)_{\varepsilon}\right] \in\left[\left(b_{\varepsilon}\right)_{\varepsilon}\right]$ se, e somente, $a_{\varepsilon}=b_{\varepsilon}$ q.s.

Demonstração. Para detalhes da prova consulte [Ver11].

Definição 1.4.3. Chamamos um filtro livre $\mathcal{F}$ sobre $I$ de selective se para cada sequência $\left(S_{n}\right)_{n \in \mathbb{N}}$ com $S_{n}^{c}:=I / S_{n} \notin \mathcal{F}$, existe $\varepsilon_{n} \in S_{n}$ tal que $\left\{\varepsilon_{n}: n \in \mathbb{N}\right\}^{c} \notin \mathcal{F}$.

Chamamos um filtro livre $\mathcal{F}$ sobre $I$ de blocked se para cada $S_{j} \subseteq I$ com $S_{j}^{c} \notin \mathcal{F}(j=1,2)$, existem $T_{j} \subseteq S_{j}$ disjuntos com $T_{j}^{c} \notin \mathcal{F}(j=1,2)$.

Chamamos um filtro livre $\mathcal{F}$ sobre $I$ de $\sigma$ blocked se para cada $S_{j} \subseteq I$ com $S_{j}^{c} \notin \mathcal{F}(j \in \mathbb{N})$, existem $T_{j} \subseteq S_{j}$ mutualmente disjuntos, com $T_{j}^{c} \notin \mathcal{F}(j \in \mathbb{N})$.

Um filtro livre $\mathcal{F}$ sobre $I$ é chamado $\aleph_{1}$-regular(resp. $\aleph_{1}$-incompleto, também chamado $\sigma$-incompleto ou $\delta$-incompleto) se existem $S_{n} \in \mathcal{F}$ tal que $\cap_{n \in \mathbb{N}} S_{n}=\emptyset\left(\right.$ resp. $\left.\cap_{n \in \mathbb{N}} S_{n} \notin \mathcal{F}\right)$. Para um ultrafiltro, $\aleph_{1}$-regular é equivalente com $\aleph_{1}$-incompleto.

Chamamos um filtro livre $\mathcal{F}$ sobre $I$ de comum se $\mathcal{F}$ é um filtro $\aleph_{1}$-regular, seletivo $e$ blocked.

Um filtro $\mathcal{F}$ é chamado Ramsey [BJ95] se para cada sequência decrescente $\left(S_{n}\right)_{n \in \mathbb{N}}$ com $S_{n} \in \mathcal{F}$, existem $\varepsilon_{n} \in S_{n}$ tal que $\left\{\varepsilon_{n}: n \in \mathbb{N}\right\} \in \mathcal{F}$. 
Observação 1.4.4. O nome blocked é devido que esta propriedade é uma obstrução de um filtro livre ser um ultrafiltro.

Com essas definições em mente vamos avante em nosso propósito de enunciar o princípio da saturação.

Lema 1.4.5. (1) Seja $\mathcal{F}$ um ultra filtro livre. Então $\mathcal{F}$ é selective se, se e somente se, é Ramsey.

(2) Se $\mathcal{F}$ é um filtro selective livre, então $\mathcal{F}$ é $\aleph_{1}$-incompleto.

Demonstração. Para detalhes da prova consulte [Ver11].

A parte (1) do Lema 1.4.5 mostra que nossa definição de filtro livre selective é consistente com o fato que um ultrafiltro Ramsey é chamado também de um selective ultrafiltro que pode ser consultado em [JW95].

Lema 1.4.6. Seja $\mathcal{F}$ um filtro livre selective e blocked. Então $\mathcal{F}$ é $\sigma$-blocked.

Demonstração. Para detalhes da prova consulte [Ver11].

Teorema 1.4.7 (Princípio da saturação). Sejam $\mathcal{F}$ um filtro comum e $X$ um conjunto interno. Então, para cada $n \in \mathbb{N}$, seja $A_{n} \subseteq X$ conjunto interno e $B_{n} \subseteq X$ tal que $X / B_{n}$ é conjunto interno, ou, $B_{n}=X$. Se $A_{1} \cap \ldots \cap A_{n} \cap B_{j} \neq \emptyset$ para cada $n, j \in \mathbb{N}$. Então $\cap_{n \in \mathbb{N}} A_{n} \cap B_{n} \neq \emptyset$.

Demonstração. Para detalhes da prova consulte [Ver11].

Corolário 1.4.8. Sejam $\mathcal{F}$ um filtro comum e $X$ um conjunto inteiro. Então para cada $n \in \mathbb{N}$, seja $A_{n} \subseteq X$ tal que $X / A_{n}$ é interno. Se $\left(A_{n}\right)_{n \in \mathbb{N}}$ tem a propriedade de interseção finita, então $\cap_{n \in \mathbb{N}} A_{n} \neq \emptyset$.

Corolário 1.4.9 (Comutação do quantificador). Sejam $\mathcal{F}$ um filtro comum e $X$ um conjunto interno. Para cada $n \in \mathbb{N}$, sejam $P_{n}\left(x, x_{n, j}\right), Q_{n}\left(x, y_{n, j}\right)$ formulas de transferência e $a_{n, j}, b_{n, j}$ constantes internas. Se $P_{n}$ fica mais forte quando $n$ cresce(ou seja, para cada $n \in \mathbb{N}$ e $x \in X$, $P_{n+1}\left(x, a_{n+1, j}\right) \Rightarrow P_{n+1}\left(x, a_{n, j}\right)$ e se $\forall n, m \in \mathbb{N}, \exists x \in X, P_{n}\left(x, a_{n, j}\right) \wedge \neg Q_{m}\left(x, b_{m, j}\right)$ então também

$$
\exists x \in X, \forall n \in \mathbb{N}, P_{n}\left(x, a_{n, j}\right) \wedge \neg Q_{n}\left(x, b_{n, j}\right)
$$

Demonstração. Para detalhes da prova consulte [Ver11].

Definição 1.4.10. Sejam $a, b \in{ }^{*} \mathbb{R}$. Então a é chamado infinitamente grande se $|a| \geq$ $n$, para cada $n \in \mathbb{N}$; a é chamado finito se $|a| \leq N$, para algum $N \in \mathbb{N}$; a é chamado infinitesimal se $|a| \leq \frac{1}{n}$, para cada $n \in \mathbb{N}$. Denotamos por $a \approx b$ se, e somente se, $a-b$ é infinitesimal. Denotamos o conjunto dos elementos finitos de ${ }^{*} \mathbb{R}$ (resp. $\left.{ }^{*} \mathbb{N}\right)$ por Fin $\left({ }^{*} \mathbb{R}\right)$ $\left(\right.$ resp.Fin $\left.\left({ }^{*} \mathbb{N}\right)\right)$ e o conjunto dos elementos infinitamente grande por ${ }^{*} \mathbb{R}_{\infty}\left(\right.$ resp. $\left.{ }^{*} \mathbb{N}_{\infty}\right)$.

Exemplo 1.4.11. Se $\mathcal{F}$ não é $\aleph_{1}$-regular, então ${ }^{*} \mathbb{N}_{\infty}=\emptyset$. De fato, se $\left[n_{\varepsilon}\right] \in{ }^{*} \mathbb{N}_{\infty}$, então $n_{\varepsilon} \in \mathbb{N}$ para cada $\varepsilon \in I$ e para cada $m \in \mathbb{N}$, existe $X_{m} \in \mathcal{F}$ tal que $n_{\varepsilon} \geq m$, para cada $\varepsilon \in X_{m}$. Como $\mathcal{F}$ não é $\aleph_{1}$-regular, existe $\varepsilon_{0} \in \cap_{m \in \mathbb{N}} X_{m}$. Então $n_{\varepsilon_{0}} \geq m$ para cada $m \in \mathbb{N}$, uma contradição. 
Este exemplo mostra que a condição de que $\mathcal{F}$ é $\aleph_{1}$-regular não pode ser deixada na afirmação do princípio da saturação.

Lema 1.4.12. Sejam $\mathcal{F}$ um filtro comum e $a \in{ }^{*} \mathbb{R}$. Se $|a| \leq m$ para cada $m \in{ }^{*} \mathbb{N}_{\infty}$, então a é finito.

Demonstração. Para detalhes da prova consulte [Ver11].

Recordamos que $\mathcal{P}_{\theta}(A)=\{X \subseteq A: X \neq \emptyset\}$.

Lema 1.4.13. Seja $A \neq \emptyset$ um conjunto não generalizado. Então ${ }^{*} \mathcal{P}_{\theta}(A)$ é o conjunto de todos os subconjuntos internos de ${ }^{*} A$.

Demonstração. Para detalhes da prova consulte [Ver11].

Vamos agora aplicar o princípio da saturação para uma das partes principais do capítulo conhecido em analise não standard como overspill e underspill.

Teorema 1.4.14. Sejam $\mathcal{F}$ um filtro comum e $A \subseteq * \mathbb{N}$ interno.

(1). (Overspill) Se A contém elementos finitos arbitrariamente grandes (ou seja, para cada $n \in$ $\mathbb{N}$, existe $m \in A$ com $m \geq n$ ), então A contém um elemento infinitamente grande.

(2).(Underspill) Se A contém arbitrariamente elementos infinitamente grandes pequenos (ou seja, para cada $\omega \in{ }^{*} \mathbb{N}_{\infty}$, existe $a \in A$ com $a \leq \omega$ ), então A contém um elemento finito.

(3).(Overspill) Se $\mathbb{N} \subseteq A$, então existe $\omega \in{ }^{*} \mathbb{N}_{\infty}$ tal que $\left\{n \in{ }^{*} \mathbb{N}_{\infty}: n \leq \omega\right\} \subseteq A$.

(4).(Underspill) $S e^{*} \mathbb{N}_{\infty} \subseteq A$, então $A \cap \mathbb{N} \neq \emptyset$.

Demonstração. Para detalhes da prova consulte [Ver11].

\subsubsection{Elementos multiplicativos}

Seja $(A,|\cdot|)$ um anel anel com uma semi-norma. Para cada a $\in \mathbf{A}$ e para todo $n \in \mathbb{N}$, temos $\left|a^{n}\right| \leq|a|^{n}$.

Definição 1.4.15. Um elemento $a \in \mathbf{A}$ é chamado potência multiplicativa se $\left|a^{n}\right|=|a|^{n}, \forall n \in$ $\mathbb{N}$.

Uma propriedade interessante é o seguinte.

Proposição 1.4.16. Os elementos do nilradical $\operatorname{rad} \mathbf{A}$ de $\mathbf{A}$ e de $\operatorname{Ker}(|\cdot|)$ são potência multiplicativa.

Definição 1.4.17. Um elemento $a \in \mathbf{A}$ é chamado multiplicativo (relativo $a|\cdot|$ ), se a $\notin$ $\operatorname{Ker}(|\cdot|)$ e $|a x|=|a| \cdot|x|, \forall x \in \mathbf{A}$.

Por consequência um elemento multiplicativo é potência multiplicativa.

Definição 1.4.18. Uma aplicação $|\cdot|: \mathbf{A} \rightarrow \mathbb{R}$, onde $\mathbf{A} \neq 0$ é chamado uma valuação (não Arqimediana) sobre A se,

(a) $|0|=0$ e $|x|>0$ para todo $x \neq 0$,

(b) $|x-y| \leq \max \{|x|,|y|\}$,

(c) $|x y|=|x||y|$.

Proposição 1.4.19. Sejam A um anel comutativo com unidade, e $|\cdot|$ denota a norma sobre $\mathbf{A}[x]$, definida nela a função grau deg. Então $|\cdot|$ é uma valuação se, e somente se, $\mathbf{A}$ é um dominio de integridade.

Prova 1.4.20. Para os detalhes da prova consulte [BGR84]. 


\subsection{Compactificação do $X(n, \mathbb{L})$}

Nesta seção introduzimos uma compactificação topológico de certos espaços devido a Khelif-Scarpalezos. Isto foi no contexto da álgebra simplificada. Os espaços em questão são os espaços de Aragona que serão definidos no capítulo sobre as álgebras de Aragona. Vamos a uma rápida introdução destes espaços. Como o nosso contexto é a álgebra full isto será feito para a álgebra full generalizando assim os resultados de Khelif-Scarpalezos e a construção clássica de Stone-Cech.

Esta seção deveria ser posta no próximo capítulo, porém como alguns resultados foram já provados por Khelif e Scarpalezos em [KS06], achamos melhor introduzir aqui. A próxima definição foi antecipada, poderia ser colocado na seção 2.2, mas o leitor pode se adinatar e fazer uma breve leitura para acompanhar esta seção.

Definição 1.5.1. Seja $A \subset X(n, \mathbb{L})$ um subconjunto, dizemos que é um conjunto generalizado de zeros $Z_{g}$, se existe $f \in \mathcal{A}(m, \mathbb{L})$ tal que $A=f^{-1}(0)=\{x \in X(n, \mathbb{L}) \mid f(x)=0\}$. Denotamos por $A=Z_{g}(f)$. Mais geralmente, se $\mathcal{B}$ é qualquer subconjunto de $\mathcal{A}(m, \mathbb{L})$, então $Z_{g}(\mathcal{B})=\cup_{f \in \mathcal{B}} f^{-1}(0)$.

Queremos relacionar os conjuntos de zeros com os filtros, então começamos introduzindo a seguinte definição.

Definição 1.5.2. Um $Z_{g}$ - filtro $\mathcal{F}$ é uma família de $Z_{g}$-conjuntos, que satisfaz as seguintes condições:

(a) Se $A$ é um $Z_{g}-$ conjunto, $B \subset A$ e $B \in \mathcal{F}$, então $A \in \mathcal{F}$.

(b) Se $A_{1}, \ldots, A_{p}$ são finitos conjuntos de $Z_{g}$, então $\bigcap_{n \leq p} A_{n} \in \mathcal{F}$.

(c) $S e \emptyset \notin \mathcal{F}$.

Com esta definição de $Z_{g}$ - filtro $\mathcal{F}$ podemos provar a seguinte proposição.

Proposição 1.5.3. Dado um $Z_{g}$ - filtro $\mathcal{F}$, a família $\mathcal{J}(\mathcal{F})=<\left\{f \in \mathcal{A}(m, \mathbb{L}) \mid f^{-1}(0) \in\right.$ $\mathcal{F}\}>$ de funções generalizadas de $\mathcal{A}(m, \mathbb{L})$ sobre $\Omega$, cujo zeros generalizados pertence a $\mathcal{F}$ é um ideal próprio.

Demonstração. Para a prova consulte [Khe].

Logo temos a seguinte definição.

Definição 1.5.4. Um ideal $\mathcal{J}$ é chamado um $Z_{g}$-ideal, se ele é da forma $\mathcal{J}(\mathcal{F})$ para algum $Z_{g}-$ filtro $\mathcal{F}$.

Definição 1.5.5. Dado um ideal $\mathcal{J}$ denotamos $\mathcal{F}(\mathcal{J})$ a família de todos $Z_{g}$ - conjuntos de elementos de $\mathcal{J}$.

Vamos provar a seguinte proposição que será útil para provarmos mais resultados relacionados.

Proposição 1.5.6. Se $\mathcal{J}$ é um ideal próprio de $\mathcal{A}(m, \mathbb{L})$, então $\mathcal{F}(\mathcal{J})$ é um $Z_{g}$ - filtro.

Demonstração. Para detalhes da prova consulte [Khe].

Agora estamos pronto para provarmos um resultado importante, mas primeiro vamos a seguinte definição. 
Definição 1.5.7. Maximal $Z_{g}$ - filtros serão chamados de $Z_{g}$ - ultrafiltros.

A seguinte proposição é o resultado para o qual estavamos criando o ambiente favorável, para chegarmos nela.

Proposição 1.5.8. Se $\mathcal{M}$ é um ideal maximal de $\mathcal{A}(m, \mathbb{L})$, então $\mathcal{F}(\mathcal{M})$ é um $Z_{g}$-ultrafiltro. Reciprocamente, se $\mathcal{F}$ é um $Z_{g}$ - ultrafiltro, então $\mathcal{J}(\mathcal{F})$ é um ideal maximal de $\mathcal{A}(m, \mathbb{L})$.

Demonstração. Consulte [Khe] para detalhes da prova.

Vamos agora provar que os ideais maximais, estão relacionados com os pontos da compactificação de $X(n, \mathbb{L})$. Com esse objetivo em mente começamos definindo.

Definição 1.5.9. Uma família $\mathcal{S}$ de subconjuntos fechados de um espaço E topológico normal, é uma familia de compactificação se satisfaz:

(1) $E \in \mathcal{S}$.

(2) Para qualquer $F \subset E$ e cada elemento de $x \notin F$ existe $F^{\prime} \in \mathcal{S}$ tal que $F \subset F^{\prime}$ e $x \notin F^{\prime}$.

(3) Para qualquer $F_{1}, F_{2} \in \mathcal{S}$ tal que $F_{1} \cap F_{2}=\emptyset$. Existe $F_{1}^{\prime}, F_{2}^{\prime} \in \mathcal{S}$ tal que $F_{1} \subset F_{1}^{\prime}, F_{2} \subset$ $F_{2}^{\prime}, \quad F_{1}^{\prime} \cup F_{2}^{\prime}=E, F_{1} \cap F_{2}^{\prime}=F_{2} \cap F_{1}^{\prime}=\emptyset$.

(4) $\mathcal{S}$ é estável por interseção finita.

(5) Se $x \in E$, então $\{x\} \in \mathcal{S}$.

Introduzindo a definição de compactificação de um conjunto, enunciamos o seguinte teorema.

Teorema 1.5.10. Sejam E espaço topológico normal, $\mathcal{H}$ uma família de compactificação de subconjuntos fechados de E, seja $\tilde{\mathcal{H}}$ o conjunto de todos $\mathcal{H}-$ filtros e seja $\hat{E}_{\mathcal{H}}$ o conjunto de $\mathcal{H}$ - ultrafiltros. Consideramos a seguinte familia de subconjutos de $\hat{E}_{\mathcal{H}}$.

$\hat{O}=\hat{E}_{\mathcal{H}} \cup\left\{G \in \hat{E}_{\mathcal{H}} \mid F \in \mathcal{H}\right.$ e $\left.F \nsubseteq G\right\}$. Ent $\tilde{a} O$

(a) Ô é a família de abertos de alguma topologia sobre $\hat{E}_{\mathcal{H}}$.

(b) A aplicação $u: E \rightarrow \hat{E}_{\mathcal{H}}$ definida por $u(x)=\{F \in \mathcal{H} \mid x \in F\}$ é injetiva e contínua.

(c) $\hat{E}_{\mathcal{H}}$ é um espaço topologico compacto e E é mergulhado densamente em algum subconjunto de $\hat{E}_{g}$.

$\hat{E}_{\mathcal{H}}$ será chamado a $\mathcal{H}$-compactificação de $E$.

Demonstração. Para detalhes da prova consulte [Khe].

Vamos provar agora que o espaço de Aragona satisfaz as condições da definição de compactificação. Mas antes provaremos o seguinte:

Lema 1.5.11. Seja $f \in \mathcal{A}(m, \mathbb{L})$. Se a é um número estritamente positivo e $\eta$ é outro número estritamente positivo, então existe $g \in \mathcal{A}(m, \mathbb{L})$ tal que $Z_{g}(g) \supseteq\left\{\left.x \in X(n, \mathbb{L})|| f(x)\right|^{2} \leq a\right\}$ $e\left\{\left.x \in X(n, \mathbb{L})|| f(x)\right|^{2} \leq a+\eta\right\} \supseteq Z_{g}(g)$. 
Demonstração. Seja $\phi$ uma função de $C^{\infty}(\mathbb{R})$ estritamente crescente tal que $\phi(x)=0$ para $x \leq a$ e tal que $x \geq a+\eta$ e $\phi(x)=1$. Então $\phi \in \mathcal{O}_{M}(\mathbb{R})$. Definam $g=\phi\left(|f|^{2}\right) \in \mathcal{A}(m, \mathbb{L})$. É facil ver que $g$ toma valor zero sobre todos os pontos $x$ generalizados tal que $|f(x)|^{2}=0$. $\operatorname{Logo} Z_{g}(g) \supseteq\left\{\left.x|| f(x)\right|^{2} \leq a\right\}$. Como $|f(x)|^{2}>a+\eta$, então que $\phi\left(|f(x)|^{2}\right)>0$, e obtemos que,

$$
\left\{\left.x \in X(n, \mathbb{L})|| f(x)\right|^{2} \leq a+\eta\right\} \supseteq Z_{g}(g) \supseteq\left\{\left.x \in X(n, \mathbb{L})|| f(x)\right|^{2} \leq a\right\} .
$$

Vamos usar o lema anterior para provar a seguinte:

Proposição 1.5.12. A familia de $Z_{g}$ de $Z_{g}$ - conjuntos satisfaz as seguintes propriedades.

(1) $X(n, \mathbb{L}) \in Z_{g}$.

(2) Para qualquer subconjunto fechado $F$ de $X(n, \mathbb{L})$ e $x \notin F$ existe $F^{\prime} \in Z_{g}$ tal que $x \notin F^{\prime}$ e $F^{\prime} \supset F$.

(3) Para qualquer $F_{1}, F_{2}$ elementos de $Z_{g}$ tais que $F_{1} \cap F_{2}=\emptyset$ existem $F_{1}^{\prime}$, $F_{2}^{\prime}$ elementos de $Z_{g}$ com a propriedade,

$$
F_{1} \subset F_{1}^{\prime}, F_{2} \subset F_{2}^{\prime}, F_{1}^{\prime} \cap F_{2}=F_{2}^{\prime} \cap F_{1}=\emptyset, F_{1}^{\prime} \cup F_{2}^{\prime}=X(n, \mathbb{L})
$$

(4) $Z_{g}$ é estável por interseções finitas.

(5) Se $x \in X(n, \mathbb{L})$, ent $\tilde{a} o\{x\} \in Z_{g}$.

Demonstração. (1) Claramente $Z_{g}(0)=X(n, \mathbb{L})$.

(2) Sejam $\psi \in \mathcal{D}\left(\mathbb{R}^{n}\right)$ com $0 \leq \psi \leq 1, \psi(0)=1$ e supp $\psi \subset B(0,1)$ e todas suas derivadas limitadas. Considerando $A \subset X(n, \mathbb{L})$ fechado e suponhamos que $\xi \notin A$. Dessa maneira, escolha $p \in \mathbb{N}$ e $\forall \varphi \in \mathcal{A}_{p}$.

Tome o conjunto $B$ com $\forall x \in B$ satisfazendo $\operatorname{dist}(x, A) \geq \alpha_{p}^{\bullet}$. Assim, definimos $f=$ $\left[\left(\varphi \rightarrow \psi_{*}(\cdot)\right)_{\varphi}\right]$, onde $\psi_{*}(\cdot)=\psi_{\min \{1, \iota(\varphi)\}}\left(\left(\cdot-\xi_{\varphi}\right) \iota(\varphi)^{-p}\right)=\psi\left(\left(\cdot-\xi_{\varphi}\right) \iota(\varphi)^{-p}\right)$. Pelo fato de $\psi$ ser de suporte compacto, temos que $f$ é moderada, e como $\xi$ possui uma única sombra então temos $f(\xi)=1$ e $f(x)=0 \forall x \in A$. Logo, $f: X(n, \mathbb{L}) \rightarrow \mathbb{L}$ é uma função que separa pontos de um conjuto fechado.

(3) Vamos supor que $F_{1}=Z_{g}(|f|)=Z_{g}\left(|f|^{2}\right)$, e $F_{2}=Z_{g}(|g|)=Z_{g}\left(|g|^{2}\right)$. Consideramos as funções $\tilde{f}=\frac{|f|^{2}}{|f|^{2}+|g|^{2}}$ e $\tilde{g}=\frac{|g|^{2}}{|f|^{2}+|g|^{2}}$. Vejamos que $Z_{g}(\tilde{f})=F_{1}$ e $Z_{g}(\tilde{g})=F_{2}$, temos

$$
F_{2}=\{x \in X(n, \mathbb{L}) \mid \tilde{f}(x)=1\}
$$

Para qualquer real $\eta>0$ menor do que $\frac{1}{4}$ consideramos agora $h_{1} \in \mathcal{A}(m, \mathbb{L})$ tal que, para qualquer,

$$
\left\{x \in X(n, \mathbb{L}) \mid \tilde{f}(x) \leq \frac{1}{2}\right\} \subset Z_{g}\left(h_{1}\right) \subset\left\{x \in X(n, \mathbb{L}) \mid \tilde{f}(x) \leq \frac{1}{2}+\eta\right\}
$$

e $h_{2} \in \mathcal{A}(m, \mathbb{L})$ tal que, 


$$
\left\{x \in X(n, \mathbb{L}) \mid \tilde{g}(x) \geq \frac{1}{2}+\eta\right\} \supset Z_{g}\left(h_{2}\right) \supset\left\{x \in X(n, \mathbb{L}) \mid \tilde{g}(x) \leq \frac{1}{2}\right\}
$$

A existencia de $h_{1}$ e $h_{2}$ pode ser deduzido, pelo Lema 1.5.11 aplicando nas funções $\tilde{f}$ e $\tilde{g}$. Coloquemos $F_{1}^{\prime}=Z_{g}\left(h_{1}\right)$ e $F_{2}^{\prime}=Z_{g}\left(h_{2}\right)$, considerando que $\tilde{f}+\tilde{g}=1$ e podemos verificar que

$$
F_{1}^{\prime} \cup F_{2}^{\prime}=X(n, \mathbb{L}), F_{1}^{\prime} \supset F_{1}, F_{2}^{\prime} \supset F_{2}, F_{1}^{\prime} \cap F_{2}=F_{2}^{\prime} \cap F_{1}=\emptyset .
$$

(4) Seja $f \in \mathcal{A}(m, \mathbb{L})$ e faça $\tilde{f}=\frac{|f|^{2}}{1+|f|^{2}}$. Então $|\tilde{f}| \leq 1$. Considerando $Z_{g}\left(f_{n}\right)=B_{n}$ e faça $B_{n}=Z_{g}\left(\tilde{f}_{n}\right)$ e definamos $g:=\sum_{n=1}^{\infty} \alpha_{n}^{\bullet}|\tilde{f}|^{2}$. Como $\left.\alpha \cdot f\right|^{2} \approx 0$, temos que $g$ converge. Assim, como $\mathcal{A}(m, \mathbb{L})$ é um espaço completo, obtemos que $g \in \mathcal{A}(m, \mathbb{L})$, $\operatorname{logo}, Z_{g}(g)=\cap Z_{g}\left(\tilde{f}_{n}\right)=\cap B_{n}$, e portanto $X(n, \mathbb{L})$ é estável sob interseções.

(5) Seja $\left(x_{\varphi}\right)_{\varphi}$ representante de $x \in \tilde{\Omega}_{c}$ e para $\varphi \in \mathcal{A}_{0}$ definamos $f_{\varphi}(\cdot)=\left(\cdot-\pi\left(x_{\varphi}\right) / \Omega\right)$. Então $f=\left[\left(\left(\cdot-\pi\left(x_{\varphi}\right) / \Omega\right)\right)_{\varphi}\right]=(\cdot-\pi(x) / \Omega)$ e segue que $Z_{g}(f)=\left[\left(\pi\left(x_{\varphi}\right)\right)_{\varphi}\right]=\pi(x) \in$ $X(n, \mathbb{L})$. Logo obtemos uma família de subconjutos fechados de $X(n, \mathbb{L})$ do espaço de Aragona que satisfaz a definição de compactificação. A conclusão que o espaço $X(n, \mathbb{L})$ tem uma compactificação segue pelo Teorema 1.5.10. 


\section{Capítulo 2}

\section{Álgebras de Aragona}

\section{$2.1 \quad$ Preliminar}

Consideramos $\overline{\mathbb{K}}$ com a topologia cortante definida no capitulo anterior. Vamos brevemente relembrar alguns conceitos e elementos introduzidas na teoria por Aragona-Juriaans, no caso da álgebra simplificada, e posteriormente estendidos, no contexto da álgebra full por Aragona-Juriaans-Garcia.

Seja $A \subset \mathcal{A}_{0}(\mathbb{K})$, e denotamos por $A^{c}$ o complemento de $A$ em $\mathcal{A}_{0}(\mathbb{K})$, por $\chi_{A}$ a função característica de $A$ com dominio $\mathcal{A}_{0}(\mathbb{K})$. Seja $\mathcal{S}=\left\{S \subset I \mid 0 \in \bar{S} \cap \bar{S}^{c}\right\}$, onde $\bar{S}$ denotao fecho topológico do conjunto $S$ em $I=] 0,1]$, e

$$
\mathcal{S}_{f}=\left\{A \subset \mathcal{A}_{0}(\mathbb{K}) \mid \forall p \in \mathbb{N}, \exists \varphi \in \mathcal{A}_{p}(\mathbb{K}),\left\{\varepsilon \mid \varphi_{\varepsilon} \in A\right\} \in \mathcal{S}\right\}
$$

Denotamos por $P_{*}\left(\mathcal{S}_{f}\right)$ o conjunto de todos os subconjuntos $\mathcal{F}$ de $P\left(\mathcal{S}_{f}\right)$ com a seguinte propriedade:

1. $\forall A \in \mathcal{S}_{f}$, temos que $A \in \mathcal{F}$ ou $A^{c} \in \mathcal{F}$.

2. Se $A, B \in \mathcal{F}$, então $A \cup B \in \mathcal{F}$.

$\mathrm{Na}$ literatura, conjuntos com estas propriedades são conhecidos como cofiltros. Se $\mathcal{F} \in$ $P_{*}\left(\mathcal{S}_{f}\right)$, denotemos por $g_{f}(\mathcal{F})$ o ideal de $\overline{\mathbb{K}}$ gerado pelo conjunto de idempotentes $\left\{\chi_{A} \quad \mid \quad A \in\right.$ $\mathcal{F}\}$. Escolhendo cada $\mathcal{F} \in P_{*}\left(\mathcal{S}_{f}\right)$, o ideal $g_{f}(\mathcal{F})$ é um ideal primo próprio, veja em [AJ01, AGJ13]. Sabemos que Inv $(\overline{\mathbb{K}})$, o grupo de unidades de $\overline{\mathbb{K}}$, é aberto e denso em $\overline{\mathbb{K}}$ e que $H=\left\{\dot{\alpha}_{r} \mid r \in R\right\}$, onde $\dot{\alpha}_{r}=(\operatorname{diam}(\operatorname{supp}(\phi)))^{r}$ ou $\dot{\alpha}_{r}=(i(\phi))^{r}$ and $i(\phi)$ denota o diametro do suporte de $\phi \in \mathcal{A}_{0}(\mathbb{K})$ é, um subgrupo de Inv $(\overline{\mathbb{K}})$. Lembremos que podemos considerar $\mathcal{G}_{f}(\Omega) \subset \mathcal{F}\left(\tilde{\Omega}_{c}, \overline{\mathbb{K}}\right), \tilde{\Omega}_{c} \subset \overline{\mathbb{K}}^{n}$.

Dado $\xi \in \tilde{\Omega}_{c}$, definamos $\nu_{\xi}: \mathcal{G}(\Omega) \longrightarrow \overline{\mathbb{K}}$ por $\nu_{\xi}(f)=f(\xi)$. Agora se $Y \subset \tilde{\Omega}_{c}$ e $I \triangleleft \overline{\mathbb{K}} e ́$ um ideal então $\mathcal{G}_{Y, I}(\Omega)=\left\{f \in \mathcal{G}(\Omega) \mid \nu_{\xi}(f) \in I, \forall \xi \in Y\right\}$ é um ideal de $\mathcal{G}(\Omega)$. Estes ideais são próprios se, e somente se, I é um ideal próprio. Considerando ideais $I \neq J \triangleleft \overline{\mathbb{K}}$ tem-se que $\mathcal{G}_{\xi, I}(\Omega) \neq \mathcal{G}_{\xi, J}(\Omega)$. Se $\xi \neq \zeta \in \tilde{\Omega}_{c}$, e se $I \triangleleft \overline{\mathbb{K}}$ então $\mathcal{G}_{\xi, I}(\Omega) \neq \mathcal{G}_{\zeta, J}(\Omega)$. Por outro lado, se $I, J \triangleleft \overline{\mathbb{K}}$ são ideais e $\xi, \zeta \in \tilde{\Omega}_{c}$ então $\xi=\zeta$ e $I=J$ se, e somente se, $\mathcal{G}_{\xi, I}(\Omega)=\mathcal{G}_{\zeta, I}(\Omega)$ ( [AJ01, AJOS08a]). Portanto um ideal $\mathcal{M} \triangleleft \mathcal{G}(\Omega)$ é maximal se, e somente se, $m=\nu_{\xi}(\mathcal{M})$ é um ideal maximal de $\overline{\mathbb{K}}$. 
Dado um ideal $\mathcal{J}$ de $\mathcal{G}(\Omega)$, o seu traço generalizado é definido por

$$
\operatorname{GTr}(\mathcal{J})=\left\{\xi \in \tilde{\Omega}_{c} \mid \nu_{\xi}(\mathcal{J}) \neq \overline{\mathbb{K}}\right\}
$$

e seu traço por $G \operatorname{Tr}(\mathcal{J}) \cap \Omega$ ([AJOS08a]). O conjunto de funções generalizadas de suporte compacto, $\mathcal{G}_{c}(\Omega)=\{f \in \mathcal{G}(\Omega) \mid \operatorname{supp}(f) \subset \subset \Omega\}$, é um ideal denso de $\mathcal{G}(\Omega)$ cujo traço generalizado é vazio ( [AJOSO8a]).

Como já mencionamos em [AJOS08a] foram provados vários resultados interessantes, entre eles o

Teorema 2.1.1 (Teorema Fundamental de $\overline{\mathbb{K}}$ [AJOS08a]). Em $\overline{\mathbb{K}}$ vale o seguinte:

1. $x \in \operatorname{Inv}(\overline{\mathbb{K}})$ se, e somente se, $Z(\hat{x}) \notin \mathcal{S}_{f}$, para todo representante $\hat{x}$ de $x$.

2. $x \notin \operatorname{Inv}(\overline{\mathbb{K}})$ se, e somente se, $\exists e \in \overline{\mathbb{K}}, e^{2}=e$ tal que $x \cdot e=0$. Em particular, se $x \in \overline{\mathbb{K}}-\{0\}$, e $x \notin \operatorname{Inv}(\overline{\mathbb{K}})$, então $x$ é um divisor de zero.

3. $x \in \operatorname{inv}(\overline{\mathbb{K}})$ se, e somente se, existe $r \in \mathbb{R}$ tal que $\alpha_{r}^{\bullet} \leq|x|$.

4. A álgebra booleana $\mathcal{B}(\overline{\mathbb{K}})=\left\{\chi_{T}: T \in \mathcal{S}_{f}\right\}$.

Seguimos agora com umas definições devido a Rosinger. Denotamos $C\left(\mathbb{R}^{n}\right)$ o conjuntos das funções continuas a valores reais sobre $\mathbb{R}^{n}$, e $\Lambda$ um conjunto infinito arbitrario. Consideramos $\left(C\left(\mathbb{R}^{n}\right)\right)^{\Lambda}$ produto cartesiano de $\Lambda$ copias $C\left(\mathbb{R}^{n}\right)$, e isto também pode ser identificado com $C\left(\Lambda \times \mathbb{R}^{n}\right)$, o conjunto das funções continuas sobre $\Lambda \times \mathbb{R}^{n}$, onde $\Lambda$ é tomado com a topologia discreta. $\left(C\left(\mathbb{R}^{n}\right)\right)^{\Lambda}$ é uma álgebra unitária comutativa sobre $\mathbb{R}$ e temos a identificação

$$
\begin{aligned}
C\left(\mathbb{R}^{n}\right) & \mapsto\left(C\left(\mathbb{R}^{n}\right)\right)^{\Lambda} \\
\psi & \mapsto u(\psi)
\end{aligned}
$$

onde $u(\psi)=\left(\psi_{\lambda} \mid \lambda \in \Lambda\right)$, com $\psi_{\lambda}=\psi, \forall \lambda \in \Lambda$. Por construção, a unidade em $\left(C\left(\mathbb{R}^{n}\right)\right)^{\Lambda} \dot{e}$ $u(1)$, onde $1 \in C\left(\mathbb{R}^{n}\right)$ denota a função constante 1 . Denotamos por $\mathcal{U}_{\Lambda}\left(\mathbb{R}^{n}\right)$ a imagem de $C\left(\mathbb{R}^{n}\right)$ em $\left(C\left(\mathbb{R}^{n}\right)\right)^{\Lambda}$ via a identificação acima, ou seja $\mathcal{U}_{\Lambda}\left(\mathbb{R}^{n}\right)=\left\{u(\psi) \mid \psi \in C\left(\mathbb{R}^{n}\right)\right\}$.

Seja $\Lambda$ um conjunto infinito, $\mathbb{K} \in\{\mathbb{R}, \mathbb{C}\}, \Omega \subset \mathbb{K}^{n}$ um subconjunto aberto e $C(\Omega)$ o anel de funções continuas tomando valores em $\mathbb{K}$. Dado uma subálgebra $\mathcal{A} \subset C(\Omega)^{\Lambda}$ e um ideal $I \triangleleft \mathcal{A}$, dizemos que o par $(\mathcal{A}, I)$ satisfaz a condição fora da diagonal se o seguinte valer

$$
I \cap\left(\mathcal{A} \cap \mathcal{U}_{\Lambda}(\Omega)\right)=\{0\}
$$

Neste caso, a álgebra quociente $\frac{\mathcal{A}}{I}$ contém uma cópia de $\mathcal{U}_{\Lambda}(\Omega)$. Estes pares aparecem no estudo de equações não lineares. As álgebras de Colombeau são exemplos de álgebras construídas desta forma.

\section{2 Álgebras de Aragona}

Vimos que existe um monomorfismo de álgebras $\kappa: \mathcal{G}(\Omega) \longrightarrow \mathcal{F}\left(\tilde{\Omega}_{c}, \overline{\mathbb{K}}\right)$ que comuta com a derivação. Por [AFJ05, Proposição 3.2], se $\|h\|<1$ então $x+h \in \tilde{\Omega}_{c}$. Sejam $I \triangleleft \overline{\mathbb{K}}$ um ideal, $\mathbb{L}=\overline{\mathbb{K}} / I$ e $\pi: \overline{\mathbb{K}} \longrightarrow \mathbb{L}$ a projeção canônica. Esta aplicação se estende de $\overline{\mathbb{K}}^{n}$ para $\mathbb{L}^{n}$, aplicando em cada coordenada, e ainda será denotado por $\pi$. 
A desigualdade generalizada de Cauchy-Schwartz e o Teorema do Valor Médio, foram provados em [AFJ05] para a álgebra simplificada. Eles foram generalizadas para a álgebra Full em [Cor]. Usaremos estes resultados livremente no que segue.

Dado $f \in \mathcal{G}(\Omega)$, denotamos $\kappa(f)$ ainda por $f$. Se $x \in \tilde{\Omega}_{c}$ e $h \in I^{n}$ é tal que $x+h \in \tilde{\Omega}_{c}$, então, pelo Teorema do Valor Médio, existe $z \in \tilde{\Omega}_{c}$ tal que

$$
f(x+h)-f(x)=\langle\nabla f(z) \mid h\rangle \in I
$$

Consequentemente, em $\mathbb{L}$, temos $\pi(f(x+h))=\pi(f(x))$.

Consideremos a álgebra de funções $\mathcal{F}\left(\mathbb{L}^{n}, \mathbb{L}\right)$, a sub-álgebra $\mathcal{A}=\mathcal{F}(X, \mathbb{L})$, com $X=\pi\left(\tilde{\Omega}_{c}\right)$ todas com a topologia quociente. Pelo que acabamos de ver acima, a aplicação $\pi$ induz um homomorfismo

$$
\begin{gathered}
\Psi: \mathcal{G}(\Omega) \longrightarrow \mathcal{A} \\
\Psi(f)(\pi(x))=\pi(f(x))
\end{gathered}
$$

No caso em que $I=m$ é um ideal maximal, tem-se que $\mathbb{L}$ é um corpo que também denotamos por $\overline{\mathbb{K}}_{m}$.

Em homenagem a um dos pioneiros da teoria, introduzimos as seguintes nomenclaturas. Seja $X(n, \mathbb{L})=\pi\left(\tilde{\Omega}_{c}\right)$ e $\mathcal{A}(m, \mathbb{L})=\mathcal{A}=\mathcal{F}(X(n, \mathbb{L}), \mathbb{L})$ a álgebra de funçôes definida em $X(n, \mathbb{L})$ e tomando valores em $\mathbb{L}$. Chamamos $X(n, \mathbb{L}) \subset \overline{\mathbb{K}}_{m}^{n}$ de um $n$-Espaço de Aragona $e$ $\mathcal{A}(n, \mathbb{L})$ de uma Álgebra de Aragona. Observemos que estes espaços dependem do ideal maximal, mas, no caso de $\mathbb{K}=\mathbb{C}$, um resultado de Steiner garante que, a menos de isomorfismo, existe um único espaço de Aragona e logo uma única álgebra de Aragona para cada dimensão $n \in \mathbb{N}$. Esses nomes foram dados devido à grande contribuição que o professor Alfredo Jorge Aragona Vallejo deu na teoria de funções generalizadas de Colombeau.

Nosso objetivo é mostrar que as distribuições de Schwartz podem ser imersas numa álgebra de Aragona. Para conseguir fazer isto, é necessário que desenvolvemos um Cálculo Diferencial sobre as álgebas de Aragona. Todorov obteve um resultado nesta direção mas a nossa abordagem não só é diferente mas também mostra que as álgebras envolvidos são quocientes de uma álgebra de Colombeau. Isto reforça a construção de Colombeau e estabelece um par com a condição fora da diagonal. É preciso também reconhecer uma construção de Khelif-Scarpalezos que, recentemente, foi compartilhado conosco. Nossos resultados não só são completos mas também vão garantir uma classificação completa dos ideias maximais de $\mathcal{G}_{f}(\Omega)$, via as álgebras de Aragona. Prosseguimos agora desenvolvendo o Cálculo Diferencial sobre as álgebras de Aragona.

Lema 2.2.1. Temos que $J=\operatorname{Ker}(\Psi)=\{f \in \mathcal{G}(\Omega): \operatorname{Im}(f) \subset m\}$. Além disso, $J$ é um ideal diferencial $\mathcal{G}(\Omega)$ e se $F \in \mathcal{G}(\Omega)$ é tal que $\frac{\partial F}{\partial x_{j}} \in J$ então existe uma constante $c \in \overline{\mathbb{K}}$ tal que $c+F \in J$. Em particular, se $n=1$, isto significa que cada $f \in J$ tem uma primitiva em $J$.

Demonstração. Suponhamos primeiro que $n=1$. Já que $m$ é fechado ([AJ01]) temos que se $f \in \operatorname{Ker}(\Psi)$ então,

$$
f^{\prime}(x)=\lim _{n \rightarrow \infty} \frac{f\left(x+\alpha_{n}\right)-f(x)}{\alpha_{n}} \in m
$$

Como toda $f \in \mathcal{G}(\Omega)$ tem uma primitiva $F$ (vide [AFJ05]), então podemos supor $0 \in \Omega$ e $F(0)=0$. Assim, lembrando que $f(x) \in m, \forall x$, tem-se que se $f \in J$ então para cada $x$ existe $c_{x}$ tal que $F(x)=F(x)-F(0)=F^{\prime}\left(c_{x}\right)(x-0)=f\left(c_{x}\right) \cdot x \in m, \forall x \in \widetilde{\Omega}_{c}$, logo $F \in J$. 
Disto segue que em geral se $f \in J$ então $\partial^{\alpha} f \in J, \forall \alpha$, multi-indice. Usando a regra de Leibniz, isto prova que $\operatorname{Ker}(\Psi)$ é um ideal diferencial de $\mathcal{G}(\Omega)$ e que toda $f \in J$ e $1 \leq j \leq n$ existe $F \in J$ tal que $f=\frac{\partial F}{\partial x_{j}} \operatorname{com} F \in J$.

Corolário 2.2.2. Se $F \in \mathcal{G}(\Omega)$ e $\alpha$ é um multi indice tal que $\frac{\partial^{\alpha} F}{\partial x^{\alpha}} \in J$ então existe uma constante $c \in \overline{\mathbb{K}}$ tal que $c+F \in J$.

Como consequência, tem-se que $\mathcal{G}(\Omega) / \operatorname{Ker}(\Psi)$ está contida numa álgebra de Aragona, por que satisfaz uma condição fora da diagonal.

Podemos ver se $f \in C^{\infty}(\Omega)$ não é nula, então, $C^{\infty}(\Omega)$ é mergulhado como uma subálgebra em $\mathcal{G}(\Omega)$, pelo fato de $f$ não ser nula segue que $\operatorname{Im}(f)$ contém unidades e portanto $f \notin$ $\operatorname{Ker}(\Psi)$. Temos que $C^{\infty}(\Omega) \cap \operatorname{Ker}(\Psi)=\{0\}$. De fato, se $f \in C^{\infty}(\Omega) \cap \operatorname{Ker}(\Psi)=\{0\}$ é não nula, então existe $x_{0} \in \Omega$ tal que $f\left(x_{0}\right) \neq 0$ temos que $f\left(x_{0}\right) \in \operatorname{Inv}(\overline{\mathbb{K}}) \cap m$, uma contradição.

Se $0 \in \Omega$ e $\delta$ é a distribuição de Dirac, então $\delta(0)=\alpha_{-1}^{\bullet} \in \operatorname{Inv}(\overline{\mathbb{K}})$ e portanto $\delta \notin$ $\operatorname{Ker}(\Psi)$. Vamos provar que $\operatorname{Ker}(\Psi) \cap D^{\prime}(\Omega)=0$ e com isso, $D^{\prime}(\Omega)$ terá uma cópia numa Álgebra de Aragona. Sendo $\Psi$ um homomorfismo de álgebras, é fácil ver que $\Psi$ é injetiva quando restrita a $D^{\prime}(\Omega)$ se, esomente se, $\Psi$ é injetiva quando restrito a $\mathcal{E}^{\prime}(\Omega)$, o subespaço de distribuição de suporte compacto. De fato, se $w \in J \cap D^{\prime}(\Omega)$ não é zero então existe um subconjunto compacto $K \subset \Omega$ tal que a restrição $\chi_{K} \cdot w \neq 0$ e $\Psi\left(\chi_{K} \cdot w\right)=\Psi\left(\chi_{K}\right) \cdot \Psi(w)=0$.

Quando $\mathbb{K}=\mathbb{R}$ temos que $\mathbb{L}=\overline{\mathbb{R}}_{m}$ é um corpo fechado real e segue que $\overline{\mathbb{C}}_{m}$ é um corpo algebricamente fechado e não depende do ideal maximal $m$, ou seja, eles são todos isomorfos. Vamos provar que estes corpos são ultra métricos onde a métrica é dado por

$$
\|\pi(\alpha)\|_{m}=\operatorname{Inf}\{\|\alpha+h\|: h \in m\}
$$

Esta ultra métrica induz a topologia quociente sobre estes corpos, e, em particular, $\pi$ é uma função Lipschitz com constante Lipschitz igual a 1. Temos ainda que $\|a b\|_{m} \leq\|a\|_{m}$. $\|b\|_{m}$.

Agora vamos provar um resultado que relaciona a norma definida sobre $\mathbb{L}$ com a ultra métrica, $\mathcal{F}$, definido sobre $m$. Para mais detalhes consulte [AJ01, Ver10], usaremos resultados contidos nestas duas referências.

Lema 2.2.3. Seja $m \triangleleft \overline{\mathbb{K}}$ um ideal maximal, $\mathbb{L}=\overline{\mathbb{K}} / m$ e $\mathcal{F}$ o ultrafiltro associado a $m$. Se $\alpha \in \overline{\mathbb{K}}$, então

$$
\|\pi(\alpha)\|_{m}=\operatorname{Inf}\left\{\left\|\alpha \cdot \chi_{A}\right\|: A \in \mathcal{F}\right\}
$$

Demonstração. Primeiro notamos que $\pi\left(\chi_{A}\right)=1, \forall A \in \mathcal{F}$. Então se $\alpha \notin m$ então $\pi\left(\alpha \cdot \chi_{A}\right)=$ $\pi(\alpha)$. Como $x=\chi_{A} x+\chi_{A^{c}} x$, então que $\|x\|=\max \left\{\left\|x \cdot \chi_{A}\right\|,\left\|x \cdot \chi_{A^{c}}\right\|\right\} \geq\left\|x \cdot \chi_{A}\right\|, \forall x \in \overline{\mathbb{K}}$. Assim, então

$$
\|\pi(\alpha)\|_{m}=\operatorname{Inf}\{\|\alpha+h\|: h \in m\} \geq \operatorname{Inf}\left\{\left\|\alpha \cdot \chi_{Z(h)}\right\|: h \in m\right\}
$$

Pelo fato que $\alpha \cdot \chi_{Z(h)}=\alpha+\alpha \cdot \chi_{Z(h)^{c}}=\alpha+h_{1}, h_{1} \in m$ temos que

$$
\operatorname{Inf}\left\{\left\|\alpha \cdot \chi_{Z(h)}\right\|: h \in m\right\} \geq \operatorname{Inf}\{\|\alpha+h\|: h \in m\}=\|\pi(\alpha)\|_{m}
$$

Isto prova que a igualdade acontece.

Corolário 2.2.4. Sejam $m \triangleleft \overline{\mathbb{K}}$ um ideal maximal, $\mathbb{L}=\overline{\mathbb{K}}_{m}, r \in \mathbb{R}, \mathbb{L}^{0}=\left\{x \in \mathbb{L}:\|x\|_{m} \leq\right.$ 1\} $e \check{\mathbb{L}}=\left\{x \in \mathbb{L}:\|x\|_{m}<1\right\}$. Então temos que 
1. Se $\pi(x)<\pi(y)$ em $\mathbb{L}$ então $\|\pi(x)\|_{m} \leq\|\pi(y)\|_{m}, \forall x, y \in \overline{\mathbb{K}}$.

2. Todos os elementos de $\mathbb{L}$ são potências multiplicativas, ou seja, $\left\|\pi\left(x^{n}\right)\right\|_{m}=\|\pi(x)\|_{m}^{n}$, $\forall n \in \mathbb{N}$ e $x \in \overline{\mathbb{K}}$.

3. $\mathbb{L}^{0}$ é um subanel $e \check{\mathbb{L}}$ é um ideal primo de $\mathbb{L}^{0}$.

4. $\pi\left(\alpha_{r}^{\bullet}\right)$ é um elemento multiplicativo de $\mathbb{L}$, ou seja, em $\mathbb{L}$ temos que $\left\|\pi\left(\alpha_{r}^{\bullet} \cdot x\right)\right\|_{m}=$ $\left\|\pi\left(\alpha_{r}^{\bullet}\right)\right\|_{m} \cdot\|\pi(x)\|_{m}, \forall x \in \overline{\mathbb{K}}$.

Demonstração. Para provar o primeiro item, podemos escolher $0<x<y$ em $\overline{\mathbb{K}}$. Para cada $A \in \mathcal{F}$ então temos que $0<x \cdot \chi_{A}<y \cdot \chi_{A}$. Tomamos o ínfimo, o resultado segue.

Para provar o segundo item usamos o lema anterior e o fato que $\left\|x^{n}\right\|=\|x\|^{n}$ em $\overline{\mathbb{K}}$.

Para o terceiro item, se $x, y \in \mathbb{L}^{0}$ então $\|x \cdot y\|_{m} \leq\|x\|_{m} \cdot\|y\|_{m} \leq 1$ e $\|x+y\|_{m} \leq$ $\max \left\{\|x\|_{m},\|y\|_{m}\right\}$, e isto mostra que $\mathbb{L}^{0}$ é um subanel de $\mathbb{L}$. Da mesma forma, segue que $\check{\mathbb{L}}$ é um subanel também, e contido em $\mathbb{L}^{0}$. Agora escolhamos $x, y \in \mathbb{L}^{0}$ tal que $x \cdot y \in \check{\mathbb{L}}$. Já que $\check{\mathbb{L}}$ é um subanel e $\mathbb{L}$ é totalmente ordenado, nós podemos supor que $0<x<y$ com isso $\|x\|_{m} \leq\|y\|_{m}$. Multiplicando por $x$, temos que $0<x^{2}<x \cdot y$. Usando o segundo item temos que $\|x\|_{m}^{2}=\left\|x^{2}\right\|_{m} \leq\|x \cdot y\|_{m}<1$. Então nós devemos ter que $x \in \check{\mathbb{L}}$, provando a primalidade deste ideal.

Para a prova do ultimo item, recordamos de [AJ01] que os elementos de $\alpha_{r}$ são elementos multiplicativos de $\overline{\mathbb{K}}$ e que $\left\|\chi_{A}\right\|=1$. Usando isto e o lema anterior temos que,

$$
\begin{gathered}
\left\|\pi\left(\alpha_{r}\right)\right\|_{m}=\operatorname{Inf}\left\{\left\|\alpha_{r} \cdot \chi_{A}\right\|: A \in \mathcal{F}\right\}=\left\|\alpha_{r}\right\| \cdot \operatorname{Inf}\left\{\left\|\chi_{A}\right\|: A \in \mathcal{F}\right\} \\
=\left\|\alpha_{r}\right\|
\end{gathered}
$$

portanto obtemos que

$$
\begin{gathered}
\left\|\pi\left(\alpha_{r} \cdot x\right)\right\|_{m}=\operatorname{Inf}\left\{\left\|\alpha_{r} \cdot x \cdot \chi_{A}\right\|: A \in \mathcal{F}\right\}=\left\|\alpha_{r}\right\| \cdot \operatorname{Inf}\left\{\left\|x \cdot \chi_{A}\right\|: A \in \mathcal{F}\right\} \\
=\left\|\alpha_{r}\right\| \cdot\|\pi(x)\|_{m}=\left\|\pi\left(\alpha_{r}\right)\right\|_{m} \cdot\|\pi(x)\|_{m}
\end{gathered}
$$

Note que o resultado provado acima não dependem se a álgebra de Colombeau é a simplificada ou a full. Segue que a norma resultante é sempre uma pm-norma e $\mathbb{L}^{0} / \check{\mathbb{L}}$ é sempre um dominio de integridade. Portanto, o resultado seguinte se aplica para todas estas álgebras.

Teorema 2.2.5. Sejam $m \triangleleft \overline{\mathbb{K}}$ um ideal maximal e $\mathbb{L}=\overline{\mathbb{K}}_{m}$. Então $\|\cdot\|_{m}$ é uma valuação de $\mathbb{L}$.

Demonstração. Para provar este resultado, aplicamos o corolário 2.2.4 e [BGR84, 1.5.3, Proposição 1], que diz que a norma para ser uma valuação é suficiente ter certos elementos multiplicativos e $\mathbb{L}^{0} / \check{\mathbb{L}}$ ser um domínio de integridade.

Usando o Cálculo Diferencial introduzido por Araogona-Juriaans, no contexto da álgebra simplificada, e generalizada para a full álgebra por Garcia, vamos introduzir um Cálculo Diferencial nas álgebras de Aragona $\mathcal{F}\left(\mathbb{L}^{n}, \mathbb{L}\right)$.

Visto que $\mathbb{L}$ é um corpo, começamos a definir uma derivação sobre $\mathbb{L}$. Dizemos que a função $f: U \subset \mathbb{L}^{n} \longrightarrow \mathbb{L}$ é diferenciável no ponto $x_{0}$ se existir um $a \in \mathbb{L}$ tal que

$$
\lim _{x \rightarrow x_{0}} \frac{f(x)-f\left(x_{0}\right)-a\left(x-x_{0}\right)}{x-x_{0}}=0
$$


Lema 2.2.6. Seja $y_{0}=\pi\left(x_{0}\right) \in X(n, \mathbb{L})$ e $f \in \mathcal{G}(\Omega)$. A função $\pi(f)$ é diferenciável em $y_{0}$.

Demonstração. Começamos a prova usando parte da prova do Teorema do Mergulho em [AFJ05, Theorem 4.1] que diz o seguinte: para qualquer $x, x_{0} \in \tilde{\Omega}_{c}$ e $h \in m^{n}$ existe uma constante $C(h)$ tal que

$$
\begin{gathered}
\left\|\pi\left(f(x+h)-f\left(x_{0}\right)-f^{\prime}\left(x_{0}\right)\left(x+h-x_{0}\right)\right)\right\|_{m} \leq \\
\left\|f(x+h)-f\left(x_{0}\right)-f^{\prime}\left(x_{0}\right)\left(x+h-x_{0}\right)\right\| \leq C\left\|x-x_{0}+h\right\|^{2}
\end{gathered}
$$

Disto, e o fato que $\|\cdot\|_{m}$ é uma valuação sobre $\mathbb{L}$, segue-se que

$$
\begin{gathered}
\left\|\pi(f)(\pi(x))-\pi(f)\left(\pi\left(x_{0}\right)\right)-\pi\left(f^{\prime}\right)\left(\pi\left(x_{0}\right)\right)\left(\pi(x)-\pi\left(x_{0}\right)\right)\right\|_{m} \leq \\
C\left\|\pi(x)-\pi\left(x_{0}\right)\right\|_{m}^{2}
\end{gathered}
$$

Finalmente podemos provar o

Teorema 2.2.7 (Teorema do Mergulho). Dado um subconjunto aberto $\Omega \subset \mathbb{R}^{n}$ existe um corpo ultra métrico $\mathbb{L}$, com a ultra métrica dado por uma valuação, um espaço de Aragona $X(n, \mathbb{L}) \subset \mathbb{L}^{n}$, uma Álgebra de Aragona $\mathcal{A}(n, \mathbb{L})$ e um monomorfismo linear

$$
\tau: D^{\prime}(\Omega) \longrightarrow C^{\infty}(X(n, \mathbb{L}), \mathbb{L}) \subset \mathcal{A}(n, \mathbb{L})
$$

tal que

$$
\tau\left(\partial^{\alpha} T\right)=D^{\alpha}(\tau(T)), \forall T \in D^{\prime}(\Omega), \forall \alpha
$$

Demonstração. Temos que $\tau=\Psi \circ \kappa$ onde $\kappa$ é a aplicação do Teorema do Mergulho [AFJ05, Theorem 4.1]. É claro que é uma aplicação linear e devemos provar que é injetiva. Como visto acima, isto significa que devemos provar que $\operatorname{Ker}(\Psi) \cap \mathcal{E}^{\prime}(\Omega)=0$. Mas se $w \in \operatorname{Ker}(\Psi) \cap \mathcal{E}^{\prime}(\Omega)$ então então $w=\partial^{\alpha} f$, para algum multi indice $\alpha$ e alguma função continua $f \in C^{0}(\Omega)$. Aplicando o Corolário 2.2.2, segue que $f \in J=\operatorname{Ker}(\Psi)$. Se $f$ não fosse zero existiria $x_{0} \in \Omega$ tal que $f\left(x_{0}\right) \neq 0$. Como $\kappa(f)\left(x_{0}\right) \in f\left(x_{0}\right)+\overline{\mathbb{K}}_{0}$ e $f\left(x_{0}\right) \in \operatorname{Inv}(\overline{\mathbb{K}}) \cap \mathbb{R}$ de norma igual a 1 , segue que $\kappa(f)\left(x_{0}\right) \in \operatorname{Inv}(\overline{\mathbb{K}})$ pois, caso contrário, existiria um idempotente não trivial $e$ tal que $\kappa(f)\left(x_{0}\right) \cdot e=0$. O que implicaria que $f\left(x_{0}\right) \cdot e \in \overline{\mathbb{K}}_{0}$, uma contradição.

$\mathrm{O}$ fato que de $\tau$ comutar com a derivação segue diretamente da definição da aplicação e do Teorema do Mergulho de [AFJ05].

A injetividade também segue dos resultados do Todorov e Vernaev [Tod06, TV08]. De fato, seja $T \in D^{\prime}(\Omega) \cap \operatorname{Ker}(\Psi)$. Segue [TV08, Definiçãa 2.5, Definição 4.1] que a imagem de $T$ em $\widehat{\mathbb{C}^{\mathcal{D}_{0}}}$ é zero. Podemos agora usar [TV08, Teorema 5.3, Teorema \%.12] para concluir que $T=0$.

O teorema permite vermos as distribuições como funções do calculo clássico. Vamos usar esse teorema para o estudo dos ideais maximais na álgebra de Colombeau $\mathcal{G}(\Omega)$ e dos idempotentes da álgebra full. As provas funcionam para as álgebra full e simplificada.

Lema 2.2.8. Sejam $\Omega \subset \mathbb{R}^{n}$ e $0 \neq T \in \mathcal{E}^{\prime}(\Omega)$ uma distribuição de suporte compacto. Então existe um $r>0$ tal que $\forall x \in \widetilde{\Omega}_{c}$ temos que $\alpha_{r}^{\bullet} \cdot T(x) \in \overline{\mathbb{K}}_{0}$.

Demonstração. Se $x=\left[\left(x_{\phi}\right)\right]$ então $T(x)=\left[\left(T\left(x_{\phi}\right)\right)\right]$, onde $T\left(x_{\phi}\right)=T \star \phi\left(x_{\phi}\right)=T\left(\phi_{x_{\phi}}\right)$ e $\phi_{x_{\phi}}(y)=\phi\left(x_{\phi}-y\right)$. Calculamos $T(x)\left(\phi_{\epsilon}\right)=T\left(x_{\phi_{\epsilon}}\right)=T \star \phi_{\epsilon}\left(x_{\phi_{\epsilon}}\right)$ usando o fato que $T=\partial^{\alpha_{0}} f$. Notamos que depois de uma mudança de variável temos que $T(x)\left(\phi_{\epsilon}\right)=(-1)^{\left|\alpha_{0}\right|} \epsilon^{-\left|\alpha_{0}\right|} \int_{W} f\left(x_{\phi_{\epsilon}}-\right.$ 
$\epsilon y) \partial^{\alpha_{0}} \phi(y) d y$ e $\epsilon^{1+\left|\alpha_{0}\right|} \cdot i(\phi)^{1+\left|\alpha_{0}\right|} T(x)\left(\phi_{\epsilon}\right)=(-1)^{\left|\alpha_{0}\right|} \cdot i(\phi)^{1+\left|\alpha_{0}\right|} \int_{W} f\left(x_{\phi_{\epsilon}}-\epsilon y\right) \partial^{\alpha_{0}} \phi(y) d y$. Assim que, $\left|\int_{W} f\left(x_{\phi_{\epsilon}}-\epsilon y\right) \partial^{\alpha_{0}} \phi(y) d y\right| \leq C(\phi)$.

Escolha uma subsequência $\left(\epsilon_{n}\right)$ tal que $x_{\phi_{\epsilon_{n}}}$ converge para o ponto $x_{0} \in W$. A última desigualdade acontece porque

$$
\int \partial^{\alpha} \phi=\int \partial^{\alpha} \phi \cdot \widehat{\delta}=\int \widehat{\partial^{\alpha} \phi} \cdot \delta=(-1)^{|\alpha|} \int x^{\alpha} \widehat{\phi} \cdot \delta=0
$$

Seguindo a Análise não-standard, para cada $x_{0} \in \overline{\mathbb{K}}^{n}$ definimos o halo de $x_{0}$ por halo $\left(x_{0}\right)=$ $\left\{x \in \overline{\mathbb{K}}^{n}: x-x_{0} \in \overline{\mathbb{K}}_{0}^{n}\right\}=x_{0}+$ halo( 0$)$. Lembramos que $\overline{\mathbb{K}}_{0}$ consiste dos elementos de $\overline{\mathbb{K}}$ que estão associados a 0 . Usando [AFJ05, Proposição 3.2] temos que se $x_{o} \in \tilde{\Omega}_{c}$ então $\operatorname{halo}\left(x_{0}\right) \subset \tilde{\Omega}_{c}$.

Todos os elementos de um espaço de Aragona são limitados porque todas suas coordenadas são limitados, pois $\widetilde{\Omega}_{c} \subset B_{1}(0)$.

Lema 2.2.9. Seja $X(n, \mathbb{L})$ um espaço de Aragona associado a $\Omega \subset \mathbb{K}^{n}$. Então

$$
X(n, \mathbb{L})=\bigcup_{x \in \Omega} h a l o(x)
$$

onde

$$
\operatorname{halo}(x)=x+\left\{y \in \mathbb{L}:\|y\|_{m}<1\right\}
$$

Demonstração. Basta considerar o caso $n=1$. Pois podemos mergulhar $\mathbb{R}$ em $\mathbb{L}$ como conjunto discreto, segue que se $b \in \mathbb{L}-\mathbb{R}$ é limitado, ou seja, $r<b<s \operatorname{com} r, s \in \mathbb{R}$, então o conjunto $A_{0}=\{x \in \mathbb{R}: x<b\}$ é limitado por acima em $\mathbb{R}$ por $s$ and $A_{1}=\{x \in \mathbb{R}: b<x\}$ é limitado por baixo em $\mathbb{R}$ por $r$. Assim, $b_{0}=S u p\left(A_{0}\right) \in \mathbb{R}$ e $b_{1}=\operatorname{Inf}\left(A_{1}\right) \in \mathbb{R}$. Claramente os elementos de $A_{1}$ são cotas superiores de $A_{0}$ e temos que $b_{0} \leq b_{1}$. Se ele não fosse igual poderia existir $n \in \mathbb{N}$ tal que $b_{0}<b_{0}+\frac{1}{n}<b_{1}$. Já que $\mathbb{L}$ é totalmente ordenado e $b \notin \mathbb{R}$ temos que $b_{0}+\frac{1}{n}<b$ ou $b<b_{0}+\frac{1}{n}$. No último caso temos que $b_{0}+\frac{1}{n} \in A_{1}$ com isso $b_{0}+\frac{1}{n} \geq$ $\operatorname{Inf}\left(A_{1}\right)=b_{1}$, uma contradição. No primeiro caso poderiamos ter que $b_{0}+\frac{1}{n} \in A_{0}$ temos que $b_{0}+\frac{1}{n} \leq \operatorname{Sup}\left(A_{0}\right)=b_{0}$, uma contradição e segue que $b_{0}=b_{1}$. Já que $b \neq b_{0} \in \mathbb{R}$ podemos supor que $b-b_{0}>0$ e que não é um infinitesimal. Neste caso, existe $n \in \mathbb{N}$ tal que $b-b_{0}>\frac{1}{n}$, e isto poderia implicar que $b_{0}+\frac{1}{n} \in A_{0}$ e consequentemente $b_{0}+\frac{1}{n} \leq \operatorname{Sup}\left(A_{0}\right)=b_{1}=b_{0}$, mais uma contradição. Isto prova que para um elemento limitado de $b \in \mathbb{L}$ existe $b_{0} \in \mathbb{R}$ tal que $b \in$ halo $\left(b_{0}\right)$. Este elemento $b_{0}$ deve ser único pois dois números reais distintos não podem estar associados a 0 , ou seja, não podem diferir por um infinitesimal. Lembrando que todos os elementos do espaço de Aragona são limitados, segue-se que o resultado está provado.

Dizemos que um elemento $f$ de uma álgebra de Aragona é limitado se $f(x)$ é limitado para todo $x \in X(n, \mathbb{L})$. Neste caso definimos,

$$
\tilde{f}(x)=\operatorname{shadow}[f(x)]
$$

onde shadow $[f(x)] \in \mathbb{R}$ é um único número real tal que $f(x) \in$ halo(shadow $[f(x)])$. Temos que $\tilde{f}=0$ se e somente se $\operatorname{Im}(f) \subset$ halo(0). Estas funções estão associadas à composição de funções generalizadas. 
Dizemos que a função $f$ é acotada se existe $r>0$ tal que $\alpha_{r}^{\bullet} \cdot f$ é limitada. O conjunto das funções limitadas forma um subanel de $\mathcal{A}(n, \mathbb{L})$. Se uma função não é acotada, dizemos que ela é ilimitada ou não acotada. Por exemplo $f_{0}=\delta$, a função Delta de Dirac, não limitada, pois $\delta(0)=\alpha_{-1}^{\bullet}$, que é uma cota superior de $\mathbb{N}$. Por outro lado, qualquer distribuição de suporte compacto, em particular $f_{0}$, é acotada pelo Lema 2.2.8. Como existem distribuição de ordem não limitada segue que (veja a prova do Lema 2.2.8) que existem funções não acotadas na Álgebras de Aragona. A existência de tais funções está relacionada à existência de ideais maximais que não são fixos, ou seja, não são da forma $\mathcal{G}_{x, I}(\Omega)$. Logo, tais ideais maximais existem. Estas idéias foram introduzidos ao se estudar os ideias de $C(X)$, onde $X$ é um espaço topológico regular.

Fixando $q=\pi(p) \in X$, o ideal maximal $\mathcal{G}_{p, m}(\Omega)$ é a imagem inversa do ideal maximal $\{f \in \mathcal{A}: \Psi(f)(p)=0\}$. Observamos que

$$
\operatorname{Ker}(\Psi)=\bigcap_{p \in \tilde{\Omega}_{c}} \mathcal{G}_{p, m}(\Omega)
$$

Embora que o $\operatorname{Ker}(\Psi) \neq(0)$, ou seja, $\tau$ não é injetivo, e com isso podemos usar Álgebras de Aragona para reduzir qualquer problema nas álgebras de Colombeau a problemas em álgebra de Aragona. Isto pode ser feito pois o Radical de Jacobson Rad $(\overline{\mathbb{K}})=0$ (veja[AJ01]).

\section{$2.3 \quad$ Filtros e Idempotentes}

Nesta seção demonstraremos a relação de filtros e idempotentes. Veja que em [Ver10] foi provado que se $P$ é um ideal primo no anel de números generalizados de Colombeau, então $\mathcal{L}=\left\{A \subset(0,1) \mid \chi_{A} \in P\right\}$ é um ultra filtro sobre $(0,1)$ contendo $J_{0}=\{] \eta, 1[: \eta \in] 0,1[\}$. Podemos fazer uma construção análoga na álgebra Full de Colombeau. Começamos definindo $\mathcal{J}_{f}=\left\{A \subset \mathcal{A}_{0}(\mathbb{K}) \mid \forall p \in \mathbb{N}, \exists \varphi \in \mathcal{A}_{p}(\mathbb{K})\right.$ tal que $\left.\left\{\varepsilon \mid \varphi_{\varepsilon} \in A\right\} \in J_{0}\right\}$.

Lema 2.3.1. Em $\overline{\mathbb{K}}$ acontece o seguinte.

1. Se $\mathcal{F} \in P_{*}\left(\mathcal{S}_{f}\right)$, então $\mathcal{L}_{f}=\left\{A \subset \mathcal{A}_{0}(\mathbb{K}): \chi_{A} \in g_{f}(\mathcal{F})\right\}=\mathcal{F} \cup \mathcal{J}_{f}$ é um co-filtro maximal sobre $\mathcal{A}_{0}(\mathbb{K})$.

2. Se $\mathcal{L}_{f}$ é um co-filtro maximal sobre $\mathcal{A}_{0}(\mathbb{K})$ contendo $\mathcal{J}_{f}$, então $\mathcal{F}=\mathcal{L}_{f} \cap \mathcal{S}_{f} \in P_{*}\left(\mathcal{S}_{f}\right)$

Demonstração. Como já notado, $g_{f}(\mathcal{F})$ é um ideal primo de $\overline{\mathbb{K}}$ segue que

$\left\{A \subset \mathcal{A}_{0}(\mathbb{K}): \chi_{A} \in g_{f}(\mathcal{F})\right\}$ é um ultra filtro. Assim $\mathcal{L}_{f}$ é um co-filtro maximal. Isto prova a primeira parte.

Já que $\mathcal{L}_{f}$ é um co-filtro maximal, ele é fechado para união finita e $\forall A \in \mathcal{S}_{f}$, temos que, $A$ ou $A^{c}$ está em $\mathcal{L}_{f}$. A prova estará completa se provarmos que $\mathcal{F}$ é fechado sob união finita. De fato, notamos que temos a união disjunta $\mathcal{L}_{f}=\left(\mathcal{S}_{f} \cap \mathcal{L}_{f}\right) \cup\left(\mathcal{J}_{f} \cap \mathcal{L}_{f}\right)$ e, claramente, $\mathcal{S}_{f} \cap \mathcal{J}_{f}=\emptyset$. Se $A \in \mathcal{S}_{f}$ então $A$ ou $A^{c} \in \mathcal{L}_{f}$ segue que $A$ ou $A^{c} \in \mathcal{L}_{f} \cap \mathcal{S}_{f}=\mathcal{F}$. Finalmente, se $A, B \in \mathcal{F}$ então $A \cup B \in \mathcal{L}_{f}$ e, já que escrevemos $\mathcal{L}_{f}$ como uma união disjunta, precisamos mostrar que $A \cup B \in \mathcal{S}_{f} \cap \mathcal{L}_{f}$. Se isso não fosse o caso, então $A \cup B \in \mathcal{J}_{f} \cap \mathcal{L}_{f}$. Disto temos que $\chi_{A}+\chi_{B}-\chi_{A \cap B}=0$. Multiplicando a última equação com $\chi_{A}$ temos que $\chi_{A}=0 \mathrm{e}$ portanto $A \in \mathcal{J}_{f}$, uma contradição.

Corolário 2.3.2. Se $P \triangleleft \overline{\mathbb{K}}$ é um ideal primo, então $g_{p}=\left\{A \subset \mathcal{A}_{0}(\mathbb{K}): \chi_{A} \in P\right\}$ é um ultra filtro sobre $\mathcal{A}_{0}(\mathbb{K})$ contendo $J_{0}$. 
Vamos determinar a álgebra booleana de $\mathcal{G}(\Omega)$. Fornecemos duas provas: a primeira usando o cálculo discontínuo e a outra usando as Álgebras de Aragona. Isto mostra a utilidade das álgebras de Aragona. Porém é na classificação dos ideias maximais de $\mathcal{G}_{f}(\Omega)$ que veremos a utilidades das álgebras de Aragona.

Teorema 2.3.3. Para um subconjunto aberto conexo $\Omega$ de $\mathbb{R}^{n}$ temos que $\mathcal{B}(\mathcal{G}(\Omega))=\mathcal{B}(\overline{\mathbb{K}})$.

Demonstração. Para provar que $\mathcal{B}(\overline{\mathbb{K}}) \subset \mathcal{B}(\mathcal{G}(\Omega))$, consideramos $\mathcal{B}(\overline{\mathbb{K}}) \subset \mathcal{G}(\Omega)$ como funções constantes.

Agora seja $f \in \mathcal{B}(\mathcal{G}(\Omega))$ um idempotente não trivial. Então, já que $f$ é função diferenciável sobre $\tilde{\Omega}_{c}$ é também um idempotente, temos que $f(x)$, é idempotente em $\overrightarrow{\mathbb{K}}$ para todo $x \in \tilde{\Omega}_{c}$. Consequentemente $f(x) \in \mathcal{B}(\overline{\mathbb{K}})$. Já que a álgebra booleana $\overline{\mathbb{K}}$ é um subconjunto discreto, $\Omega$ é conexo e $f$ é diferenciável, segue que $f$ é constante sobre $\tilde{\Omega}_{c}$. Portanto existe $T \in \mathcal{S}_{f}$, tal que $f=\chi_{T}$.

Vamos provar o mesmo resultado usando as Álgebras de Aragona. Seja $f \in \mathcal{B}(\mathcal{G}(\Omega))$ um idempotente não trivial e escolha pontos, $x_{1} \neq-x_{0}$ em $\tilde{\Omega}_{c}$. Então existe um ideal maximal $m \triangleleft \mathcal{G}(\Omega)$ tal que $f\left(x_{0}\right)-f\left(x_{1}\right) \notin m$ e então $\tau(f)$ é um idempotente não constante, mas os $i d e m p o t e n t e s$ em $\mathbb{L}$ são 0 e 1 , e como $\tau(f)$ é contínua, temos uma contradição.

\subsection{Traços de Ideais}

Relembramos a desigualdade generalizada de Cauchy-Schwartz e o Teorema do Valor Médio já mencionado na seção anterior. Seja $\Omega$ um subconjunto aberto conexo de $\mathbb{R}^{n} e$ $f \in \mathcal{G}(\Omega)$. Para $x, y \in \tilde{\Omega}_{c}$ existe $z \in \tilde{\Omega}_{c}$ tal que $f(x)-f(y)=\langle\nabla f(z) \mid x-y\rangle$ e para $x, y \in \overline{\mathbb{K}}$ temos que $|\langle x, y\rangle| \leq[x]_{2}[y]_{2}$, onde $x=\left(x_{1}, \cdots, x_{n}\right)$ e $[x]_{2}=\left(\sum_{i=1}^{n}\left[x_{i}\right]\right)^{\frac{1}{2}}$.

Proposição 2.4.1. Seja $\Omega$ um subconjunto aberto de $\mathbb{R}^{n}$ e $\xi \in \tilde{\Omega}_{c}$. A função $\nu_{\xi}: \mathcal{G}(\Omega) \longrightarrow$ $\overline{\mathbb{K}}, \nu_{\xi}(f)=f(\xi)$, é um epimorfismo contínua de $\overline{\mathbb{K}}$ - álgebras.

Demonstração. Já que os elementos de $\overline{\mathbb{K}}$ são identificados como funções constantes de $\mathcal{G}(\Omega)$ temos que $\nu_{\xi}$ é epimorfismo que é claramente $\overline{\mathbb{K}}$-linear.

Para provar a continuidade, vamos usar os resultados sobre a topologia cortante de $\mathcal{G}(\Omega)$ (vide também [AFJ05, AGJ13]). Já que $\nu_{\xi}$ é um homomorfismo, apenas temos que provar sua continuidade em 0 . Seja $\left(f_{n}\right) \subset \mathcal{G}(\Omega)$ que converge para zero, e já que $\xi=\left[\left(\xi_{\varphi}\right)\right]$ é compactamente suportado, existe $\Omega_{1} \subset \subset \Omega$ e $p \in \mathbb{N}$ tal que $\xi_{\varphi} \in \Omega_{1}, \forall \varphi \in \mathcal{A}_{p}(\mathbb{K})$. Agora o homomorfismo restrição $j: \mathcal{G}(\Omega) \longrightarrow \mathcal{G}\left(\Omega_{1}\right)$ é contínua, dado um elemento $W_{l, r}^{\beta}$ de base filtrada de $\mathcal{G}\left(\Omega_{1}\right)$ existe $n_{0} \in \mathbb{N}$ tal que $j\left(f_{n}\right) \in W_{l, r}^{\beta}, \forall n \geq n_{0}$. Assim, $\left|\partial^{\sigma} f_{n}(z)\right| \leq \alpha_{r}^{\bullet}, \forall z \in$ $\tilde{\Omega}_{1}, \forall \sigma \leq \beta$. Em particular, $\left|f_{n}(\xi)\right| \leq \alpha_{r}^{\bullet}, \forall n \geq n_{0}$ e portanto $f_{n}(\xi) \in V_{r}[0], \forall n \geq 0$, que prova que $f_{n}(\xi)$ converge para 0 .

A convexidade de ideais afirma que se $\mathcal{J}$ é um ideal de $\overline{\mathbb{R}}$ e $x, y \in \overline{\mathbb{R}}$ então $x \in \mathcal{J}$ se, e somente se, $|x| \in \mathcal{J}$. Portanto, se $|y| \leq|x|$, então $y \in \mathcal{J}$ e no caso $\mathbb{K}=\mathbb{R}, x \in \mathcal{J}$ e $0 \leq y \leq x$, então $y \in \mathcal{J}$ (veja [AJOSO8a]).

Teorema 2.4.2. Sejam $\xi, \zeta \in \tilde{\Omega}_{c}, z=[\xi-\zeta]_{2}, \mathcal{I}, \mathcal{J} \triangleleft \overline{\mathbb{K}}$ ideais e $\mathcal{L} \triangleleft \mathcal{G}(\Omega)$ um ideal.

1. Se $z \in \mathcal{J}$, então $\mathcal{G}_{\xi, \mathcal{J}}(\Omega)=\mathcal{G}_{\zeta, \mathcal{J}}(\Omega)$

2. $\mathcal{J}$ é primo e $z \in \mathcal{J}$ se, e somente se, $\mathcal{G}_{\xi, \mathcal{J}}(\Omega)=\mathcal{G}_{\zeta, \mathcal{J}}(\Omega)$

3. $\operatorname{Tr}(\mathcal{L})=\operatorname{Tr}(\overline{\mathcal{L}})$ e $G \operatorname{Tr}(\mathcal{L})=G \operatorname{Tr}(\overline{\mathcal{L}})$ 
4. Se $x_{0} \in G \operatorname{Tr}(\mathcal{L})$ e $L=\nu_{x_{0}}(\mathcal{L})$, então $\tilde{\Omega}_{c} \cap\left(x_{0}+L^{n}\right) \subset G \operatorname{Tr}(\mathcal{L})$, onde $x_{0}+L^{n}=$ $\left\{x_{0}+x \mid x \in L^{n}\right\}$.

5. Se $\mathcal{I} \neq \mathcal{J}$ então $\mathcal{G}_{\xi, \mathcal{I}}(\Omega) \neq \mathcal{G}_{\xi, \mathcal{J}}(\Omega)$

Demonstração. Seja $f \in \mathcal{G}_{\xi, \mathcal{J}}(\Omega)$. Pela proposição anterior, existe $z \in \tilde{\Omega}_{c}$ tal que $f(\xi)-$ $f(\zeta)=\langle\nabla f(z), \xi-\zeta\rangle$. Então temos, $|f(\xi)-f(\zeta)|=|\langle\nabla f(z), \xi-\zeta\rangle| \leq[\nabla f(z)]_{2}[\xi-\zeta]_{2}$. Pela convexidade de ideais e a hipótese implica que $f(\zeta) \in \mathcal{J}$. Consequentemente, $\mathcal{G}_{\xi, \mathcal{J}}(\Omega)=$ $\mathcal{G}_{\zeta, \mathcal{J}}(\Omega)$, assim prova o primeiro item.

Suponhamos $\mathcal{J}$ primo e seja $\hat{f}(\epsilon, x):=\left\langle x-\xi_{\epsilon}, x-\xi_{\epsilon}\right\rangle$. Então obviamente é uma função moderada e temos que $f \in \mathcal{G}(\Omega)$. Claramente $f(\xi)=0$ temos que $f \in \mathcal{G}_{\xi, J}(\Omega)$. Assim $f(\zeta)=[\xi-\zeta]_{2}^{2}=z^{2} \in \mathcal{J}$, e pela primalidade de $\mathcal{J}$ temos que $z \in \mathcal{J}$. Já pelo item anterior mostra a reciproca, o segundo item está provado.

Seja $\mathcal{I}$ um ideal de $\mathcal{G}(\Omega)$ e $x_{0} \in G \operatorname{Tr}(\mathcal{I})$ se $x_{0} \notin G \operatorname{Tr}(\overline{\mathcal{I}})$ então existe um elemento $f \in \overline{\mathcal{I}}$ tal que $f\left(x_{0}\right)=1$. Escolhamos $\left(\left(f_{n}\right)\right) \subset \mathcal{I}$ convergindo para $f$. A continuidade de $\nu_{x_{0}}$ implica que $\left(\nu_{x_{0}}\left(f_{n}\right)\right)$ converge para $\nu_{x_{0}}(f) \in \overline{\mathbb{K}}$. Já que $\operatorname{Inv}(\overline{\mathbb{K}})$ é aberto em $\overline{\mathbb{K}}$ e $\nu_{x_{0}}(f)=1 \in \operatorname{Inv}(\overline{\mathbb{K}})$, existe $n_{0} \in \mathbb{N}$ tal que $f_{n}\left(x_{0}\right) \in \operatorname{Inv}(\overline{\mathbb{K}}), \forall n \geq n_{0}$. Disto segue que $x_{0} \notin G \operatorname{Tr}(\mathcal{I})$, uma contradição. Assim, prova que $G \operatorname{Tr}(\mathcal{I}) \subset G \operatorname{Tr}(\overline{\mathcal{I}})$. Já que a reciproca é clara, o terceiro item está provado.

Seja $x_{0} \in \operatorname{GTr}(\mathcal{L}), L=\nu_{x_{0}}(\mathcal{L})$ e $x \in \tilde{\Omega}_{c} \cap\left(x_{0}+L^{n}\right)$. Escrevamos $x=x_{0}+h, h=$ $\left(h_{1}, \cdots, h_{n}\right), h_{i} \in L, \forall i=1, . ., n$. Pela a escolha de $x_{0}$, temos que $L \neq \overline{\mathbb{K}}$. Escolhendo $f \in \mathcal{L}$, como antes, existe $z \in \tilde{\Omega}_{c}$ tal que $f(x)-f\left(x_{0}\right)=\left\langle\nabla f(z), x-x_{0}\right\rangle=\langle\nabla f(z), h\rangle$. Assim $\left|f(x)-f\left(x_{0}\right)\right|=\left|\left\langle\nabla f(z), x-x_{0}\right\rangle\right| \leq[\nabla f(z)]_{2}\left[x-x_{0}\right]_{2}$. Pela convexidade de ideal e pelo afto que $h=x-x_{0} \in L^{n}$, temos que $f(x)-f\left(x_{0}\right) \in L$. Consequentemente, $f(x) \in L$ e $\operatorname{assim} \nu_{x}(\mathcal{L}) \subset L \neq \overline{\mathbb{K}}$. Logo, $x \in G \operatorname{Tr}(\mathcal{L})$. E portanto $\tilde{\Omega}_{c} \cap\left(x_{0}+L^{n}\right) \subset G \operatorname{Tr}(\mathcal{L})$

Para provar o último item, seja $f \in \mathcal{G}_{\xi, \mathcal{J}}(\Omega) \cap \overline{\mathbb{K}}$. Então $f$ é uma função constante e $\operatorname{assim} f=f(\xi) \in \mathcal{J}$. Segue que $\mathcal{G}_{\xi, \mathcal{J}}(\Omega) \cap \overline{\mathbb{K}}=\mathcal{J}$.

Teorema 2.4.3. Sejam $\mathcal{J}, \mathcal{M}$ ideais de $\mathcal{G}(\Omega)$. Então temos:

1. $\operatorname{GTr}(\mathcal{J})$ é fechado.

2. Se $\mathcal{M}$ é maximal, $\xi \in G \operatorname{Tr}(\mathcal{M})$ e $m=\nu_{\xi}(\mathcal{M})$. Então

a. $\mathcal{M} \cap \overline{\mathbb{K}}=m$.

b. $\nu_{x}(\mathcal{M})=m$ e $\mathcal{M}=\mathcal{G}_{x, m}(\Omega)$ para todo $x \in G \operatorname{Tr}(\mathcal{M})$;

c. $\tilde{\Omega}_{c} \cap\left\{\xi+h \mid h \in m^{n}\right\}=G \operatorname{Tr}(\mathcal{M})$

Demonstração. 1. Consideramos uma sequência $\left(\xi_{n}\right) \subset G \operatorname{Tr}(\mathcal{J})$ tal que $\xi_{n} \longrightarrow \xi$. Se $\xi \notin G \operatorname{Tr}(\mathcal{J})$, então $\nu_{\xi}(\mathcal{J})=\overline{\mathbb{K}}$ e existe $f \in \mathcal{J}$ tal que $f(\xi)=1$. Pela continuidade de $f$, temos que $f\left(\xi_{n}\right) \longrightarrow f(\xi)=1$. Já que $\operatorname{Inv}(\overline{\mathbb{K}})$ é um aberto, existe $n_{0} \in \mathbb{N}$ tal que $f\left(\xi_{n}\right) \in \operatorname{Inv}(\overline{\mathbb{K}}) \forall n>n_{0}$. Portanto, $\xi_{n} \notin G \operatorname{Tr}(\mathcal{J})$, uma contradição.

(a) Seja $f \in \mathcal{M} \cap \overline{\mathbb{K}}$. Então $f=f(\xi)=\nu_{\xi}(f) \in m$. Por outro lado, se $m \neq \mathcal{M} \cap \overline{\mathbb{K}}$, existe $f_{0} \in m$ mas $f_{0} \notin \mathcal{M}$, por maximalidade de $\mathcal{M}$, temos que $f_{0} \cdot \mathcal{G}(\Omega)+\mathcal{M}=\mathcal{G}(\Omega)$. Aplicando $\nu_{\xi}$ obtemos que $\nu_{\xi}\left(f_{0} \cdot \mathcal{G}(\Omega)+\mathcal{M}\right)=\nu_{\xi}(\mathcal{G}(\Omega))=\overline{\mathbb{K}}$. Como $\nu_{\xi}\left(f_{0} \cdot \mathcal{G}(\Omega)+\mathcal{M}\right)=$ $\nu_{\xi}\left(f_{0} \cdot \mathcal{G}(\Omega)\right)+\nu_{\xi}(\mathcal{M})=f_{0} \cdot \overline{\mathbb{K}}+m=m \neq \overline{\mathbb{K}}$, uma contradição.

Prosseguimos mostrando que $m$ é maximal. De fato, se isto não fosse o caso, então existe $x_{0} \in \overline{\mathbb{K}}$ tal que $x_{0} \cdot \overline{\mathbb{K}}+m \neq \overline{\mathbb{K}}$. A maximalidade de $\mathcal{M}$ nos dar $x_{0} \cdot \mathcal{G}(\Omega)+\mathcal{M}=\mathcal{G}(\Omega)$. Devido ao paragrafo anterior, obtemos que $x_{0} \cdot \overline{\mathbb{K}}+m=\overline{\mathbb{K}}$, uma contradição. 
(b) Seja $x \in G \operatorname{Tr}(\mathcal{J})$. Então,foi provado que $\nu_{x}(\mathcal{M})=\mathcal{M} \cap \overline{\mathbb{K}}=m$. Supondo que $\mathcal{G}_{x, m}(\Omega)$ não está contido em $\mathcal{M}$ podemos escolher $f_{0} \in \mathcal{G}_{x, m}(\Omega)$, com $f_{0} \notin \mathcal{M}$. Pela maximalidade de $\mathcal{M}$ temos que $f_{0} \cdot \mathcal{G}(\Omega)+\mathcal{M}=\mathcal{G}(\Omega)$. Aplicando $\nu_{x}$ a esta equação, obtemos que $\overline{\mathbb{K}}=\nu_{x}\left(f_{0}\right) \cdot \overline{\mathbb{K}}+m \subset m \cdot \overline{\mathbb{K}}+m=m$, uma contradição. Portanto $\mathcal{G}_{x, m}(\Omega) \subset \mathcal{M}$. Já que o primeiro é ideal maximal, temos a igualdade.

(c) Para provar o último item, notamos que, pelo teorema anterior, $\tilde{\Omega}_{c} \cap\left\{\xi+h \mid h \in m^{n}\right\} \subset$ $G \operatorname{Tr}(\mathcal{M})$. Sejam $\left.x \in G \operatorname{Tr}(\mathcal{M}), h=x-\xi=\left(h_{1}, \cdots, h_{n}\right)\right)$ e $z=[x-\xi]_{2}$. Pelo que já foi provado, temos que $\mathcal{G}_{x, m}(\Omega)=\mathcal{M}=\mathcal{G}_{\xi, m}(\Omega)$. Como $\mathcal{M}$ é maximal, então primo, segue pelo segundo item do Teorema 2.4.2 que $z^{2} \in m$. Já que $\left|h_{1}\right| \leq z^{2}$, segue por convexidade de ideais que $h_{i} \in m, \forall i$. Assim $h \in m^{\mathbf{n}}$ e $x=\xi+h \in \xi+m^{\mathbf{n}}$. Logo, usando o item 4 do Teorema 2.4 .2 finaliza a prova.

Corolário 2.4.4. Seja $\mathcal{M} \triangleleft \mathcal{G}(\Omega)$ um ideal maximal. Então

1. $\operatorname{Tr}(\mathcal{M})$ tem no maximo um elemento;

2. $\operatorname{Tr}(\mathcal{M})$ não é vazio se, e somente se $m=\mathcal{M} \cap \overline{\mathbb{K}}$ é um ideal maximal e existe um único $\xi \in \Omega$ tal que $\mathcal{M}=\mathcal{G}_{\xi, m}(\Omega)$;

3. $\operatorname{GTr}(\mathcal{M})$ é não vazio se, e somente se, $m=\mathcal{M} \cap \overline{\mathbb{K}}$ é um ideal maximal e existe $\xi \in \tilde{\Omega}_{c}$ tal que $\mathcal{M}=\mathcal{G}_{\xi, m}(\Omega)$;

4. Se $\mathcal{M}$ é denso então $G \operatorname{Tr}(\mathcal{M})=\emptyset$.

Demonstração. 1. Seja $x, y \in \operatorname{Tr}(\mathcal{M}) \subset \Omega$. Pelo item 2.c do teorema anterior temos que $x-y \in m^{n}$, onde $m=\mathcal{M} \cap \overline{\mathbb{K}}$ é maximal. Se $x \neq y$ então uma das coordenadas de $x-y \in \mathbb{K}$ é um numero real pertencente a $m$, uma contradição já que $m$ é um ideal próprio de $\overline{\mathbb{K}}$.

2. Para provar o segundo item, notamos que se $\operatorname{Tr}(\mathcal{M}) \neq \emptyset$ então, pelo item anterior, ele é um elemento único. Logo pelo segundo item do teorema anterior dado, temos a conclução.

3. Foi provado no último Teorema 2.4.3.

4. Se $\mathcal{M}$ é maximal e denso então ele não pode ser fechado. Suponhamos que $\operatorname{GTr}(\mathcal{M}) \neq$ $\emptyset$. Assim, pelo teorema anterior, $\mathcal{M}=\mathcal{G}_{x, m}(\Omega)$, para $x \in G \operatorname{Tr}(\mathcal{M})$. Já que a aplicação $\nu_{x}$ é contínua e ideais maximais de $\overline{\mathbb{K}}$ são fechados, segue que $\mathcal{M}$ é fechado, contradizendo o fato que é um ideal próprio.

Seja $\mathcal{M}=\mathcal{G}_{x_{0}, m}(\Omega) \triangleleft \overline{\mathbb{K}}$ com $m \triangleleft \overline{\mathbb{K}}$ um ideal maximal. Dado $f \in \mathcal{G}(\Omega)$ e $g=f-f\left(x_{0}\right)$ temos que $g\left(x_{0}\right)=0 \in m$ com isso $g \in \mathcal{M}$. Assim $f+\mathcal{M}=f\left(x_{0}\right)+\mathcal{M}$, com $f\left(x_{0}\right) \in \overline{\mathbb{K}}$. Isto prova que a classe residual de corpos $\frac{\mathcal{G}(\Omega)}{\mathcal{M}} \cong \frac{\overline{\mathbb{K}}}{m}$. Isto prova que, se $\mathcal{M}$ for maximal com traço generalizada não vazio, então todos os quocientes $\frac{\mathcal{G}(\Omega)}{\mathcal{M}}$ são isomorfos. Vamos chamar este tipo de ideais maximais de ideais maximais fixos. Se um ideal maximal não é fixo vamos chamar-lo de um ideal maximal livre. Então um ideal maximal é fixo se em alguma Álgebra de Aragona a interseção dos conjuntos de zeros de seus elementos da sua projeção é um ponto. Notamos que isto é similar à ideia clássica de ideal maximal em $C(X)=\{f: X \longrightarrow \mathbb{R}$ : $f$ continuas $\}$, onde $X$ é um espaço topológico compacto. Isto é exatamente o que foi sugerido [AJOS08a] e que motivou esta parte da tese. Isto é claramente uma afirmação sobre Z-filtros 
maximais em tais espaços. No nosso caso, um espaço de Aragona não é compacto, já que contém algum aberto $\Omega \subset \mathbb{R}$ como subconjunto discreto. Logo o processo de compactificação é mais complicado e foi explicado nas prelimináres. Pode ser provado que este processo não é a compactificação de Stone-Cech.

Finalizando, mostramos como descrever todos os ideais maximais de $\mathcal{G}_{f}(\Omega)$. Seja $\mathcal{M} \triangleleft$ $\mathcal{G}_{f}(\Omega)$ um ideal maximal e seja $I=\mathcal{M} \cap \overline{\mathbb{K}}$. Temos então que existem um monomorfismo de $\overline{\mathbb{K}} / I$ com $\mathcal{G}_{f}(\Omega) / \mathcal{M}$ e logo $\overline{\mathbb{K}} / I$ é um anel de integridade. Concluimos que I é um ideal primo. Logo existe um filtro maximal $\mathcal{F}$ tal que $I=g_{f}(\mathcal{F})$. Consideramos o anel de frações $\mathbb{L}=Q\left(\overline{\mathbb{K}} / g_{f}(\mathcal{F})\right)$. Seguimos o roteiro da segunda seção para obter um homomorfismo de álgebras de $\mathcal{G}_{f}(\Omega)$ em um espaço de funções $\mathcal{F}(X, \mathbb{L})$, onde $X$ é a projeção de $\widetilde{\Omega}_{c}$ em $\mathbb{L}^{n}$. Temos também uma cópia de $D^{\prime}(\Omega)$ em $\mathcal{F}(X, \mathbb{L})$. Podemos fazer agora um processo de compactificação e parametrizar os ideias maximais usando os ideais primos $g_{f}(\mathcal{F})$ e os pontos da compactificação.

Para entendermos as relações das álgebras de Colombeau e Aragona, fazemos o seguinte gráfico que comuta:
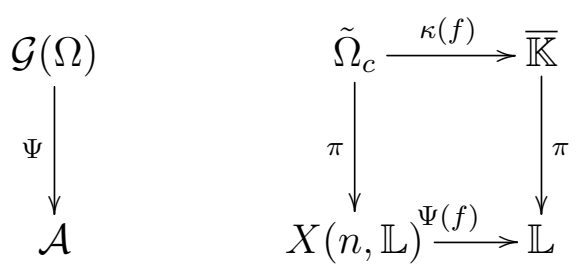


\section{Capítulo 3}

\section{Conjuntos Internos}

\subsection{Teoria dos Conjuntos Internos}

A teoria de conjuntos internos e funções internas vem crescendo ao longo das últimas décadas, em [OK99] aparece a noção de função interna na álgebra de Colombeau. Neste artigo eles caracterizam os elementos em $\mathcal{G}(\Omega)$ por seus pontos generalizados, onde $\Omega \subseteq \mathbb{R}^{n}$ é um aberto e sabendo que $\mathcal{G}(\Omega)$ é a álgebra simplificada de Colombeau. Essa caracterização é muito importante, pois a mesma diz que o dominio de um elemento $\mathcal{G}(\Omega)$ é exatamente $\tilde{\Omega}_{c}$.

Melhorando nossa investigação e alargando o conjunto $\mathcal{G}(\Omega)$ para $\mathcal{G}_{f}(\Omega)$, no trabalho feito por Eduard A. Nigsch em [Nig13] ele fez um trabalho similar de Oberguggenberger, caracterizou os elementos de $\mathcal{G}_{f}(\Omega)$ (na álgebra plena de Colombeau), verificando também que os elementos de $\mathcal{G}_{f}(\Omega)$ também são funções internas cujo domínio é $\tilde{\Omega}_{f, c}$.

Apesar da teoria de conjuntos internos aparecer na análise não standard como pode ser visto em [Ver11], é no trabalho de Aragona e Juriaans em [AFJO12] que surge as primeiras propriedades de conjuntos internos na álgebra simplificada de Colombeau, ao qual os autores chamaram de membranas como pode ser verificado em [AFJO12]. Neste trabalho eles desenvolveram cálculo diferencial e integral nas álgebras de Colombeau simplificada e provaram várias propriedades de membranas.

Acrescentando com a teoria de conjuntos internos, Oberguggenberger e Vernaeve no artigo [OV08], provaram várias propriedades de conjuntos internos e funções internas, estendendo a noção de membranas da álgebra simplificada de Colombeau. No trabalho [OV08] tem como objetivo principal em provar interseções e uniões de conjuntos internos.

Motivado com o trabalho de Oberguggenger e Vernaeve, nós ficamos atraídos em investigar as propriedades de conjuntos internos e funções internas na álgebra plena de Colombeau. Com isso em mente nosso principal objetivo neste capitulo é provar várias propriedades de conjuntos internos e funções internas para a álgebra plena de Colombeau.

Vamos fazer algumas definições estendendo as definições feitas em [OV08]. Começamos com a seguinte definição.

Vamos considerar E um espaço vetorial localmente convexo. Seja $\left(p_{i}\right)_{i \in I}$ uma família de semi - normas gerando a topologia de E. Então definimos $\mathcal{G}_{E}:=\mathcal{E}_{M}(E) / \mathcal{N}_{E}$, onde temos

$$
\begin{gathered}
\mathcal{E}_{M}=\left\{\left(u_{\varphi}\right)_{\varphi} \in E^{\mathcal{A}_{0}}:(\forall i \in I), \exists p \in \mathbb{N}, \forall \varphi \in \mathcal{A}_{p}, \exists \eta \in(0,1) \text { tal que } \forall \varepsilon \leq\right. \\
\left.\eta, \text { temos } p_{i}\left(u_{\varphi}\right) \leq \varepsilon^{-p} \iota(\varphi)^{-p}\right\} \\
\mathcal{N}_{E}=\left\{\left(u_{\varphi}\right)_{\varphi} \in E^{\mathcal{A}_{0}}:(\forall i \in I), \quad \forall m \in \mathbb{N}, \exists q \in \mathbb{N}, \forall \varphi \in \mathcal{A}_{q}, \exists \eta \in(0,1) \text { tal que } \forall \varepsilon \leq\right. \\
\left.\eta, \text { temos } p_{i}\left(u_{\varphi}\right) \leq \varepsilon^{p} \iota(\varphi)^{p}\right\}
\end{gathered}
$$


Vamos considerar $E$ um espaço vetorial normado. Seja $U(\Omega)$ o conjunto dos pares $(\varphi, x) \in$ $\mathcal{A}_{0}\left(\mathbb{R}^{n}\right) \times \Omega$ satisfazendo supp $\varphi+x \subseteq \Omega$. Agora vamos para definição com espaços vetoriais.

Definição 3.1.1. $\mathcal{E}_{M, f}(E)=\left\{\left(u_{\varphi}\right)_{\varphi} \in E^{\mathcal{A}_{0}} \mid \forall \varphi \in \mathcal{A}_{0}(E), \exists \eta>0, e \forall \varepsilon<\eta\right.$ : temos $\left(\varphi_{\varepsilon}, u_{\varphi_{\varepsilon}}\right) \in U(E), \quad e \exists p \in \mathbb{N} ; \forall \varphi \in \mathcal{A}_{p}(E), \varepsilon \leq \eta$, temos $\left.\left\|u_{\varphi_{\varepsilon}}\right\|=O\left(\varepsilon^{-p}\right)\right\}$

$\mathcal{N}(E)=\left\{\left(u_{\varphi}\right)_{\varphi} \in E^{\mathcal{A}_{0}}: \forall m \in \mathbb{N}, \exists q \in \mathbb{N} ; \forall \varphi \in \mathcal{A}_{q}(E) ; \varepsilon \leq \eta\right.$, temos, $\left.\left\|u_{\varphi_{\varepsilon}}\right\|=O\left(\varepsilon^{m}\right)\right\}$

Então definimos $\mathcal{G}_{E}=\frac{\mathcal{E}_{M, f}(E)}{\mathcal{N}(E)}$. Assim, chamamos os elementos de $\mathcal{E}_{M, f}(E)$ e $\mathcal{N}(E)$ de rede moderadas e nulas respectivamente. $\mathcal{G}_{E}$ é chamado o espaço de Colombeau construído sobre $E$, no caso quando $E=\mathbb{R}$, ou $E=\mathbb{C}$, escrevemos $\tilde{\mathbb{R}}_{f}$ e $\tilde{\mathbb{C}}_{f}$ para $\mathcal{G}_{f}$.

Quando $\Omega$ é um aberto de $\mathbb{R}^{d}$ e $E=C^{\infty}(\Omega)$, o espaço $\mathcal{G}_{f}$ é uma álgebra diferencial, chamada de álgebra plena de Colombeau de funções generalizadas, e denotado por $\mathcal{G}_{f}(\Omega)$.

No nosso trabalho vamos considerar $E=\mathbb{R}^{n}$ a menos que faça menção contraria. Portanto tomamos um subconjunto $\Omega \subseteq \mathbb{R}^{n}$ e começamos a definir um conjunto imaginário que na maior parte do nosso trabalho será desenvolvido neste conjunto. Seja $\Omega \subseteq \mathbb{R}^{n} e$ $I_{\eta}\left(\right.$ onde $\left.\left.\left._{\eta}=\right] 0, \eta\right]\right)$, então definamos:

Definição 3.1.2. $\Omega_{M, f}=\left\{\left(x_{\varphi}\right)_{\varphi} \in \Omega^{\mathcal{A}_{0}}: \exists p \in \mathbb{N}, \forall \varphi \in \mathcal{A}_{p}, \exists c=c_{\varphi}>0, \varepsilon \in I_{\eta},\left|x_{\varphi_{\varepsilon}}\right| \leq\right.$ $\left.c \varepsilon^{-p}\right\}$.

Então definimos uma relação de equivalência definida por,

$\left(x_{\varphi}\right)_{\varphi} \sim\left(y_{\varphi}\right)_{\varphi} \Leftrightarrow \exists p \in \mathbb{N}, \exists \gamma \in \Gamma ; \forall \varphi \in \mathcal{A}_{q}$ para $\forall q \geq p, \exists C=C_{\varphi}>0$ e $\forall \varepsilon \in I_{\eta}$, temos $\left|x_{\varphi_{\varepsilon}}-y_{\varphi_{\varepsilon}}\right| \leq C \varepsilon^{\gamma(q)-p}$.

Então definimos o conjunto $\tilde{\Omega}_{f}:=\Omega_{M, f} / \sim$. O conjunto suportado compactamente é por definição,

$\tilde{\Omega}_{c}=\left\{\tilde{x} \in \tilde{\Omega}_{f}:\right.$ existem representantes $\left(x_{\varphi}\right)_{\varphi}, \exists K \subset \subset \Omega$ compacto, $\exists p \in \mathbb{N}, \forall \varphi \in$ $\left.\mathcal{A}_{p}, \forall \varepsilon \in I_{\eta} ; x_{\varphi_{\varepsilon}} \in K,\right\}$.

Claro que se a propriedade de $\tilde{\Omega}_{c}$ acontece para um representante de $\tilde{x} \in \tilde{\Omega}$, então acontece para cada representante. Para $\Omega=\mathbb{K}$, temos $\tilde{\mathbb{K}}=\overline{\mathbb{K}}$. Portanto temos a identificação canônica $\tilde{\mathbb{K}}^{n}=\overline{\mathbb{K}}^{n}$ e $\tilde{\mathbb{K}}_{c}=\overline{\mathbb{K}}_{c}$.

Definição 3.1.3. Um subconjunto $A$ de $\tilde{\Omega}_{f}$ é chamado conjunto interno se existe uma rede $\left(A_{\varphi}\right)_{\varphi} \subset \Omega^{\mathcal{A}_{0}}$ de subconjuntos de $\Omega \subset E$ tal que

$$
A=\left\{u \in \tilde{\Omega}_{f} \mid \exists \text { representantes }\left(u_{\varphi}\right)_{\varphi} \text { de } u, \exists p \in \mathbb{N}, \forall \varphi \in \mathcal{A}_{p}(E) \text { com } u_{\varphi} \in A_{\varphi}\right\}
$$

Denotamos por $A=\left[\left(A_{\varphi}\right)_{\varphi}\right]$.

Agora vamos considerar $E=\mathbb{R}^{d}$ e trabalharemos com $\mathbb{R}^{n}$ a menos se mencionarmos o contrário. Seja $\Omega \subset \mathbb{R}^{n}$, então definimos,

Definição 3.1.4. Sejam $\left(M_{\varphi}\right)_{\varphi} \subset \Omega^{\mathcal{A}_{0}(n)}$ e $P_{f}(\Omega)_{M}$ uma familia de subconjuntos $M_{\varphi} \subset \Omega \subseteq$ $\mathbb{R}^{n}$, tais que:

1. Existe $K \subset \subset \Omega$ compacto $e \exists N \in \mathbb{N}$ tal que $\forall \varphi \in \mathcal{A}_{N}(n)$ acontece $M_{\varphi} \subset \subset K$.

2. A função característica de $M_{\varphi}$ é integrável à Lebesgue.

Qualquer elemento de $P_{f}(\Omega)_{M}$ é chamado de pré-membrana de dimensão n, ou uma, $n$-pré-membrana em $\Omega$. 
Definição 3.1.5. Sejam $\left(M_{\varphi}\right)_{\varphi},\left(M_{\varphi}^{\prime}\right)_{\varphi} \subset P_{f}(\Omega)_{M}$. Dizemos que $\left(M_{\varphi}\right)_{\varphi}$ é equivalente ao $\left(M_{\varphi}^{\prime}\right)_{\varphi}$, e denotamos por $\left(M_{\varphi}\right)_{\varphi} \sim\left(M_{\varphi}^{\prime}\right)_{\varphi}$, se existir uma função nula $\left(\Psi_{\varphi}\right)_{\varphi} \in \mathcal{N}_{f}\left(\Omega ; \mathbb{R}^{n}\right)$ tal que a função $\phi$ definida sobre $\mathcal{A}_{0}(n) \times \Omega \operatorname{por} \phi(\varphi, x)=x+\Psi_{\varphi}(x)$ tal que $\Psi\left(\varphi, M_{\varphi}\right)=M^{\prime} \varphi$, $\forall \varphi \in \mathcal{A}_{0}$.

Agora com essas definições podemos começar a enunciar os resultados sobre conjuntos internos, para construir tais resultados vamos usar a topologia dado por $V_{r}\left[x_{0}\right]=\{x \in$ $\left.\overline{\mathbb{K}}|| x-x_{0} \mid \leq \alpha_{r}^{\bullet}\right\}$ e $\beta=\left\{V_{r}[0] \mid r \in \mathbb{R}\right\}$ que pode ser consultado em [AFJ' 09].

Proposição 3.1.6. Seja $A=\left[\left(A_{\varphi}\right)_{\varphi}\right]$ um conjunto interno de $\tilde{\Omega}_{f}$, então $A=\left\{u \in \tilde{\Omega}_{f} \mid \operatorname{dist}(u, A)=\right.$ 0 , ou $\left.\operatorname{seja}\left(d\left(u_{\varphi} ; A_{\varphi}\right)\right)_{\varphi} \in \mathcal{N}\right\}$.

Demonstração. Por hipótese temos $\left(d\left(u_{\varphi} ; A_{\varphi}\right)\right)_{\varphi} \in \mathcal{N}$ então existe $p_{1} \in \mathbb{N}, \exists \gamma_{1} \in \Gamma, \forall \varphi \in$ $\mathcal{A}_{p_{1}}(n), \exists C_{1}>0, \exists \eta_{1}>0$ tal que $\forall \varepsilon<\eta_{1}$, temos;

$$
d_{\varphi_{\varepsilon}}=i n f_{a_{\varphi_{\varepsilon}} \in A_{\varphi_{\varepsilon}}}\left|u_{\varphi_{\varepsilon}}-a_{\varphi_{\varepsilon}}\right| \leq C_{1} \varepsilon^{\gamma_{1}(q)-p_{1}}, \forall q \geq p_{1} .
$$

Veja que $\forall k \geq 0$ e $\forall \varphi \in \mathcal{A}_{k}(n), \exists \eta>0 ; \forall \varepsilon<\eta$ tal que temos $\left|e^{-\frac{1}{\iota\left(\varphi_{\varepsilon}\right)}}\right|<\frac{1}{2^{k}}$, e segue que, $\left(e^{-\frac{1}{\imath(\varphi)}}\right)_{\varphi}$ é nula. Assim,

existe $p_{2} \in \mathbb{N}, \exists \gamma_{2} \in \Gamma, \forall \varphi \in \mathcal{A}_{p_{2}}(n), \exists C_{2}>0, \exists \eta_{2}>0 ; \forall \varepsilon<\eta_{2}$, temos $\left|e^{-\frac{1}{u\left(\varphi_{\varepsilon}\right)}}\right|<$ $C_{2} \varepsilon^{\gamma_{2}(q)-p_{2}}<1, \forall q \geq p_{2}$.

Seja $p=\max \left\{p_{1}, p_{2}\right\}, \gamma=\max \left\{\gamma_{1}, \gamma_{2}\right\}, C=\max \left\{C_{1}, C_{2}\right\}$ e $\eta=\min \left\{\eta_{1}, \eta_{2}\right\}$, e escolha que $\forall \varphi \in \mathcal{A}_{p}(n)$ existe um $a_{\varphi}^{\prime} \in A_{\varphi}$ tal que $d\left(u_{\varphi}, a_{\varphi}^{\prime}\right) \leq d_{\varphi}+e^{-\frac{1}{\imath(\varphi)}}$, então $\forall \varepsilon<\eta$, acontece $d\left(u_{\varphi_{\varepsilon}}, a_{\varphi_{\varepsilon}}^{\prime}\right) \leq C \varepsilon^{\gamma(q)-p}, \forall q \geq p$. Consequentemente $\left(u_{\varphi}\right)_{\varphi} \sim\left(a_{\varphi}^{\prime}\right)_{\varphi}$, segue que $\left\{u \in \tilde{\Omega}_{c, f}(n) \mid \operatorname{dist}(u, A)=0\right.$, ou seja $\left.\left(d\left(u_{\varphi} ; A_{\varphi}\right)\right)_{\varphi} \in \mathcal{N}\right\} \subseteq A$. A outra inclução é óbvia.

Corolário 3.1.7. Seja A um conjunto interno. Então $\left[\left(A_{\varphi}\right)_{\varphi}\right]=\left[\left(\overline{A_{\varphi}}\right)_{\varphi}\right]$.

Definição 3.1.8. Dizemos que $A \subseteq \tilde{\Omega}_{f}$ é $A$-J limitado se existir $r \in \mathbb{R}$, tal que $\forall u \in A$, verifica-se $[u]_{2} \leq \alpha_{-r}^{\bullet}$.

Definição 3.1.9. Seja $\left(A_{\varphi}\right)_{\varphi} \subset \Omega^{\mathcal{A}_{0}}$ uma rede de subconjuntos de $\Omega$. Definimos o supremo de uma rede por sup $|v|_{v \in\left[\left(A_{\varphi}\right)_{\varphi}\right]}:=b \in \overline{\mathbb{R}}_{f}$ tal que existem representantes $\left(b_{\varphi}\right)_{\varphi}$ de $b, \forall \varphi \in \mathcal{A}_{0}(n)$ temos que $\sup _{v_{\varphi} \in A_{\varphi}}\left|v_{\varphi}\right| \leq b_{\varphi}$.

Definição 3.1.10. Dizemos que uma rede $\left(A_{\varphi}\right)_{\varphi} \subset \Omega^{\mathcal{A}_{0}}$ é $A$-J limitada se existir $r \in \mathbb{R}$ tal que $\sup _{v \in\left[\left(A_{\varphi}\right)_{\varphi}\right]}|v| \leq \alpha_{-r}^{\bullet}$.

Temos o seguinte exemplo.

Exemplo 3.1.11. Sejam $\Omega=\mathbb{R}^{2}$, e $A_{\varphi}=B(0, \iota(\varphi))$, então $\operatorname{Vol}(A)=\left[\varphi \rightarrow \operatorname{Vol}\left(A_{\varphi}\right)\right]=$ $\left[\varphi \rightarrow \pi \iota(\varphi)^{2}\right]=\pi\left[\varphi \rightarrow \iota(\varphi)^{2}\right]=\pi \alpha_{2}^{\bullet}$

Logo obtemos a medida do conjunto interno, chamada de bolas generalizadas.

Proposição 3.1.12. Cada $A \subset \tilde{\Omega}_{f}$ conjunto interno é fechado na topologia $A$ - $J$ (topologia natural de Colombeau).

Demonstração. Seja $u \in \bar{A} / A$ e suponhamos que $u_{n} \in A$ tal que $\lim _{n \rightarrow \infty} \operatorname{dist}\left(u_{n}, u\right)=0$. Então $\exists n_{0} ; \forall n \geq n_{0}$, temos $\operatorname{dist}\left(u_{n}, u\right) \in \mathcal{N}$.

Sabemos que $\operatorname{dist}\left(u_{n}, A\right)=\left[\varphi \rightarrow i n f_{v \in A_{\varphi}}\left|u_{n, \varphi}-v\right|\right]=0 \in \mathcal{N}$. Assim, $\forall n \geq n_{0}$ segue que $\operatorname{dist}(u, A) \leq \operatorname{dist}\left(u_{n}, u\right)+\operatorname{dist}\left(u_{n}, A\right) \operatorname{logo} \operatorname{dist}(u, A) \in \mathcal{N}$. 
Antes de provarmos o próximo resultado vamos usar o seguinte lema, que permite $\overline{\mathbb{R}}_{f}$ ter uma relação de ordem parcial.

Lema 3.1.13. Dado $x \in \overline{\mathbb{R}}_{f}$ são equivalentes.

1. (a) cada representante $\hat{x}$ de $x$ satisfaz a condição:

$\exists N \in \mathbb{N}$ tal que para todo $b>0$ e para todo $\varphi \in \mathcal{A}_{N}, \exists \eta=\eta(b, \varphi) \in I$ verifica-se

$$
\hat{x}\left(\varphi_{\varepsilon}\right) \geq-\varepsilon^{b}, \forall \varepsilon<I_{\eta}
$$

2. (b) existe um representante $\hat{x}$ de $x$ satisfazendo (a).

3. (c) existe um representante $x_{*}$ tal que $x_{*}(\varphi) \geq 0$ para todo $\varphi \in \mathcal{A}_{0}$;

4. (d) existe um $N \in \mathbb{N}$ e um representante $x_{*}$ de $x$ tal que $x_{*}(\varphi) \geq 0, \forall \varphi \in \mathcal{A}_{N}$.

Prova 3.1.14. Veja em $\left[A F J^{+} 09\right]$.

Agora com esse lema anterior iremos aplicar em alguns resultados. Sejam $s<r$ com $s, r \in \mathbb{R}$ pelo lema anterior condição (a) implica que $\alpha_{r}^{\bullet} \leq \alpha_{s}^{\bullet}$.

Lema 3.1.15. Seja $A \subset \tilde{\Omega}_{f}$ conjunto interno que é $A$-J limitado. Então A tem representantes $A$-J limitados.

Demonstração. Como $A$ é conjunto interno, então para $u \in A, \exists q \in \mathbb{N}, \forall \varphi \in \mathcal{A}_{q}(n)$, e $\exists\left(u_{\varphi}\right)_{\varphi}$ representante de $u$ tal que $u_{\varphi} \in A_{\varphi}$.

Existe um $r \in \mathbb{R}$ e $\forall u \in A$ implica que $\exists\left(u_{\varphi}\right)_{\varphi}$ representante de $u$ tal que $[u]_{2} \leq \alpha_{-r}^{\bullet}$. Então construímos o conjunto,

$$
\tilde{A}_{\varphi}=\left\{u \in \tilde{\Omega}_{f} \mid[u]_{2} \leq \alpha_{-r-1}^{\bullet}\right\} \cap A_{\varphi} .
$$

Então $\tilde{A}_{\varphi} \neq \emptyset$ pois pelo Lema $3.1 .13 \alpha_{-r}^{\bullet} \leq \alpha_{-r-1}^{\bullet}$, e concluímos que $\left[\left(\tilde{A}_{\varphi}\right)_{\varphi}\right] \subseteq A$.

Reciprocamente como $A$ é um conjunto interno, por definição para $u \in A$, existe $q \in$ $\mathbb{N}, \forall \varphi \in \mathcal{A}_{q}(n)$, e $\exists\left(u_{\varphi}\right)_{\varphi}$ representante de $u$ tal que $u_{\varphi} \in A_{\varphi}$.

Por outro lado veja que $\exists N \in \mathbb{N}$ tal que para todo $\varphi \in \mathcal{A}_{N}(n)$, e pelo Lema 3.1.13 verifica-se $\alpha_{-r}^{\bullet} \leq \alpha_{-r-1}^{\bullet}$.

Seja $p^{\prime}=\max \{q, N\}, \forall \varphi \in \mathcal{A}_{p^{\prime}}(n)$ e $u \in A$ implica que $\exists\left(u_{\varphi}\right)_{\varphi}$ representante de $u$ e $[u]_{2} \leq \alpha_{-r-1}^{\bullet} \operatorname{com} u_{\varphi} \in A_{\varphi}$ consequentemente temos que $u_{\varphi} \in \tilde{A}_{\varphi}$. Portanto $A \subseteq\left[\left(\tilde{A}_{\varphi}\right)_{\varphi}\right]$.

Com este lema conseguimos provar o próximo resultado.

Proposição 3.1.16. Sejam $A, B \subset \tilde{\Omega}_{f}$ conjuntos internos não vazios e $A$ é $A$-J limitado. Então $\left\{[u-v]_{2} \mid u \in A, v \in B\right\}$ atinge o minimo em $\tilde{\mathbb{R}}$.

Demonstração. Por hipótese $A$ é conjunto interno, então $\exists p_{1} \in \mathbb{N}, \forall \varphi \in \mathcal{A}_{p_{1}}(n)$ com $u \in A$ existe um $\left(u_{\varphi}\right)_{\varphi}$ representante de $u$ tal que $u_{\varphi} \in A_{\varphi}$.

Sabemos também que $B$ é conjunto interno, então $\exists p_{2} \in \mathbb{N}, \forall \varphi \in \mathcal{A}_{p_{2}}(n) \operatorname{com} v \in B$ existe um $\left(v_{\varphi}\right)_{\varphi}$ representante de $v$ tal que $v_{\varphi} \in B_{\varphi}$.

Agora seja $p=\max \left\{p_{1}, p_{2}\right\}$ e $\forall \varphi \in \mathcal{A}_{p}(n)$, temos $u_{\varphi} \in A_{\varphi}$ e $v_{\varphi} \in B_{\varphi}$. Escolhemos $u_{\varphi}^{\prime} \in A_{\varphi}$ e $v_{\varphi}^{\prime} \in B_{\varphi}$ de modo que

$\left|u_{\varphi}^{\prime}-v_{\varphi}^{\prime}\right| \leq i n f_{a \in A_{\varphi}, b \in B_{\varphi}}|a-b|+e^{-\frac{1}{\imath(\varphi)}}$.

Como $\left(u_{\varphi}\right)_{\varphi}$ é moderado e $B$ é não vazio segue que $\left(v_{\varphi}\right)_{\varphi}$ é moderado. Logo tomando os representantes o resultado segue $\left[u^{\prime}-v^{\prime}\right]_{2}=i n f_{\tilde{a} \in A, \tilde{b} \in B}|\tilde{a}-\tilde{b}|+\left[\left(e^{-\frac{1}{\iota(\varphi)}}\right)_{\varphi}\right]$. 
Construímos um exemplo ao qual mostramos que a união de conjuntos internos nem sempre é interno, que enunciaremos logo a seguir, sendo assim precisamos de um elemento para que possamos fazer união de conjuntos internos que sejam internos. Tendo em vista isso precisamos do seguinte lema.

Lema 3.1.17. Seja $A \in \tilde{\Omega}_{f}$. Então temos o conjunto, $\operatorname{interl}(A)=\left\{\sum_{j=1}^{m} \chi_{S_{j}} a_{j} \mid a_{j} \in A\right.$, e $S_{1}, \ldots, S_{m} \in \mathcal{A}_{0}(n)$ uma partição de $\left.\mathcal{A}_{0}(n)\right\}$.

Se A é um conjunto interno, então interl $(A)=A$.

Demonstração. Sejam $u, v \in A$, e $B \subset S_{f}$. Então, por definição de conjunto interno,

$\exists p_{1} \in \mathbb{N}$ e $\forall \varphi \in \mathcal{A}_{p_{1}}(n)$, com $u_{\varphi} \in A_{\varphi}$, também $\exists p_{2} \in \mathbb{N}$ e $\forall \varphi \in \mathcal{A}_{p_{2}}(n), v_{\varphi} \in A_{\varphi}$.

Consideramos $p=\max \left\{p_{1}, p_{2}\right\}$ para um representante $\left(w_{\varphi}\right)_{\varphi} \in \operatorname{interl}(A)$, temos que $w_{\varphi}=\chi_{B} u_{\varphi}+\chi_{B^{c}} v_{\varphi}$

Assim, $\forall \varphi \in \mathcal{A}_{p}(n)$ só pode acontecer o seguinte, $u_{\varphi} \in A_{\varphi}$, ou, $v_{\varphi} \in A_{\varphi}$. Logo $\operatorname{interl}(A)=$ $A$.

Proposição 3.1.18 (Uniões de conjuntos internos). Sejam $A, B \subseteq \tilde{\Omega}_{f}$ conjuntos internos não vazios. Então $\left[\left(A_{\varphi} \cup B_{\varphi}\right)_{\varphi}\right]=\operatorname{interl}(A \cup B)=\left\{\chi_{S} a+\chi_{S^{c}} b \mid a \in A\right.$ e beB, com $S \subset$ $\left.\mathcal{A}_{0}(n)\right\}$

Demonstração. Seja $u \in\left[\left(A_{\varphi} \cup B_{\varphi}\right)_{\varphi}\right]$. Então $u=\left[\left(u_{\varphi}\right)_{\varphi}\right]$ e segue por definição de conjunto interno que: $\exists p \in \mathbb{N} ; \forall \varphi \in \mathcal{A}_{p}(n)$, temos $u_{\varphi} \in A_{\varphi} \cup B_{\varphi}$.

Então definimos $S=\left\{\varphi \in \mathcal{A}_{p}(n) \mid u_{\varphi} \in A_{\varphi}\right\}$. Assim,

$$
\begin{aligned}
& \hat{u}=\left\{\begin{array}{lll}
u_{\varphi}, & \text { se } & \varphi \in S, \\
a_{\varphi} & \text { se } & \varphi \in S^{c}
\end{array}\right. \\
& \hat{v}=\left\{\begin{array}{lll}
b_{\varphi}, & \text { se } & \varphi \in S, \\
u_{\varphi}, & \text { se } & \varphi \in S^{c} .
\end{array}\right.
\end{aligned}
$$

Consideramos $\chi_{S} \hat{u}+\chi_{S^{c}} \hat{v}$ e só pode ocorrer $u_{\varphi} \in A_{\varphi} \operatorname{com} \chi_{S} \hat{u}+\chi_{S^{c}} \hat{v} \in \operatorname{interl}(A \cup B)$. Então $\left[\left(A_{\varphi} \cup B_{\varphi}\right)_{\varphi}\right] \subseteq \operatorname{interl}(A \cup B)$. A recíproca é aplicando o lema anterior.

Com esse exemplo que mostramos a seguir fica evidente a importancia do resultado anterior.

Exemplo 3.1.19. Consideramos as sequências na representação decimal $0, a_{0}, a_{1}, \ldots, a_{i}, \ldots$ onde $a_{i} \in\{0, \ldots, 9\}$ e o conjunto interno $A=\{$ dízimas períodicas formada por 1 e 5$\}$, $B=\left[\left(B_{i}\right)_{i}\right] \operatorname{com} B_{0}=\{0\} e$

$B_{i}=\{$ números com representação finita $\}$. Então perguntamos a união $A \cup B$ é um conjunto interno? Se for teremos que interl $(A \cup B)=A \cup B$, e segue que $0,1,5 \in \operatorname{interl}(A \cup B)$ portanto sendo $p=015015$... concluímos que $p \in A \cup B$ que é uma contradição pois $p \notin A \cup B$.

Proposição 3.1.20 (Inclusões de conjuntos internos). Sejam $A \subseteq \tilde{\Omega}_{f}$ conjunto interno não vazio e suponhamos que $A$ tem representantes $\left(A_{\varphi}\right)_{\varphi} A$-J limitado. Então $B=\left[\left(B_{\varphi}\right)_{\varphi}\right]$ conjunto interno. Então

$$
A \subseteq B \Leftrightarrow\left(\delta_{\varphi}\right)_{\varphi}:=\left(\sup _{u \in A_{\varphi}} d\left(u, B_{\varphi}\right)\right)_{\varphi} \in \mathcal{N} .
$$


Demonstração. Suponhamos que $\left(\delta_{\varphi}\right)_{\varphi}:=\left(\sup _{u \in A_{\varphi}} d\left(u, B_{\varphi}\right)\right)_{\varphi} \in \mathcal{N}$, então pela Proposição 3.1.6 temos $A \subseteq B$.

Suponhamos que $A \subseteq B$, e que $\forall q \in \mathbb{N}$ existe uma $\varphi \in \mathcal{A}_{q}(n)$ tal que $\delta_{\varphi} \notin \mathcal{N}$. Então $\forall p \in \mathbb{N}, \forall \gamma \in \Gamma ; \exists q \geq p$ e $\exists \varphi \in \mathcal{A}_{q}(n), \forall C>0, \forall \eta>0$ com $\exists 0<\varepsilon_{0}<\eta$ tal que satisfaz,

$$
\delta_{\varphi_{\varepsilon_{0}}} \geq C \varepsilon_{0}^{\gamma(q)-p}
$$

podemos escolher uma sequência de $C_{n}>0$ e $\left(\varepsilon_{n}\right)_{n \in \mathbb{N}} \operatorname{com} \lim _{n \rightarrow \infty} \varepsilon_{n}=0$ tal que $0<\varepsilon_{n}<\eta$ acontece

$$
(*) d\left(u_{\varphi_{\varepsilon_{n}}}, B_{\varphi_{\varepsilon_{n}}}\right) \geq C \varepsilon_{n}^{\gamma(q)-p} .
$$

Então definimos o conjunto $T_{1}(\varphi)=\left\{\varepsilon_{n} \mid(\varphi)_{\varepsilon_{n}}\right.$ que satisfaz $\left.(*)\right\}$ e $T_{2}=\left\{\varphi \mid \varepsilon_{n} \in T_{1}\right\}$. Como $T_{1}(\varphi) \in \mathcal{S}$, então $T_{2} \in S_{f}$.

Veja que $u_{\varphi_{\varepsilon_{n}}} \in A_{\varphi_{\varepsilon_{n}}}$ e como $\left(A_{\varphi}\right)_{\varphi}$ é uma rede $A$ - $J$ limitada, existe $t \in \mathbb{R}$ tal que para $u \in A$ satisfaz

$$
\left|u_{\varphi_{\varepsilon}}\right| \leq \varepsilon^{-t} \iota(\varphi)^{-t}
$$

Definamos para $\varepsilon \in\left\{\varepsilon_{n} \mid n \in \mathbb{N}\right\}$ por $v_{\varphi_{\varepsilon_{n}}}:=u_{\varphi_{\varepsilon_{n}}}$ satisfazendo $\left(^{*}\right)$, e para $\varepsilon \notin\left\{\varepsilon_{n} \mid n \in \mathbb{N}\right\}$ como sendo $v_{\varphi_{\varepsilon}}:=u_{\varphi_{\varepsilon}}$ satisfazendo (3.1). Logo obtemos $v_{\varphi} \in A_{\varphi}$ e $\left(v_{\varphi}\right)_{\varphi}$ é moderado com $d\left(u_{\varphi_{\varepsilon_{n}}}, B_{\varphi_{\varepsilon_{n}}}\right) \geq C \varepsilon_{n}^{\gamma(q)-p}$.

Vejamos que $d=\left[\left(d\left(u_{\varphi}, B_{\varphi}\right)\right)_{\varphi}\right] \in \overline{\mathbb{R}}_{f}$ e como $A$ é $A$ - $J$ limitado estendemos $\left(d\left(u_{\varphi_{\varepsilon_{n}}}, B_{\varphi_{\varepsilon_{n}}}\right)\right)$ para $\left(d\left(v_{\varphi_{\varepsilon}}, B_{\varphi_{\varepsilon}}\right)\right)$. Por outro lado usando a relação de ordem de $\overline{\mathbb{R}}_{f}$, temos que $0<\chi_{T_{2}} d \leq d$, o que implica $d \in \overline{\mathbb{R}}_{f}^{*}$, por isso $A$ não está contido em $B$ absurdo. Logo $A \subseteq B$.

Corolário 3.1.21. Sejam $\left(A_{\varphi}\right)_{\varphi}$ e $\left(B_{\varphi}\right)_{\varphi}$ duas redes de subconjuntos de $\mathbb{R}^{n} A$-J limitados $\operatorname{com}\left[\left(A_{\varphi}\right)_{\varphi}\right] \neq \emptyset e\left[\left(B_{\varphi}\right)_{\varphi}\right] \neq \emptyset, A, B \subseteq \mathbb{R}^{n}$ e denotamos por $d_{H}(A, B)=\max \left(\sup _{x \in A} d(x, B)\right.$, $\left.\sup _{x \in B} d(x, A)\right)$ a distância de Hausdorff. Então $\left[\left(A_{\varphi}\right)_{\varphi}\right]=\left[\left(B_{\varphi}\right)_{\varphi}\right] \Leftrightarrow\left(d_{H}\left(A_{\varphi}, B_{\varphi}\right)_{\varphi}\right) \in \mathcal{N}$.

Corolário 3.1.22. Sejam $A \subseteq B$ tal que $\left(\delta_{\varphi}\right)_{\varphi} \in \mathcal{N}$. Então para qualquer rede nula $\left(\nu_{\varphi}\right)_{\varphi} \in$ $\mathbb{R}^{\mathcal{A}_{0}(n)}$, com $\nu_{\varphi}>0, \forall \varphi \in \mathcal{A}_{0}(n)$, temos que $A=\left[\left(\tilde{A}_{\varphi}\right)_{\varphi}\right]$ com,

$$
\tilde{A}_{\varphi}=\left\{u \in \mathbb{R}^{n}: d\left(u, A_{\varphi}\right) \leq \delta_{\varphi}+\nu_{\varphi}\right\} \cap B_{\varphi} .
$$

Demonstração. Seja $A \subseteq B$. Então $\left(\delta_{\varphi}\right)_{\varphi} \in \mathcal{N}$, e temos que

$$
\begin{gathered}
\exists p \in \mathbb{N}, \exists \gamma \in \Gamma ; \forall \varphi \in \mathcal{A}_{p}(n), \exists C>0, \exists \eta>0, \forall \varepsilon<\eta ; \\
d_{\varphi_{\varepsilon}}:=d\left(u_{\varphi_{\varepsilon}}, B_{\varphi_{\varepsilon}}\right)=i n f_{v_{\varphi_{\varepsilon}} \in B_{\varphi_{\varepsilon}}}\left|u_{\varphi_{\varepsilon}}-v_{\varphi_{\varepsilon}}\right| \leq C \varepsilon^{\gamma(q)-p}, \forall q \geq p .
\end{gathered}
$$

Escolhamos $\forall \varphi \in \mathcal{A}_{p}(n)$ um $v_{\varphi}^{\prime} \in B_{\varphi}$ tal que $d\left(u_{\varphi}, v_{\varphi}^{\prime}\right) \leq d_{\varphi}+\nu_{\varphi}$, então $\left(v_{\varphi}^{\prime}\right)_{\varphi}$ é outro representante de $\left(u_{\varphi}\right)_{\varphi}$, obtemos

$$
\tilde{A_{\varphi}}=\left\{u \in \mathbb{R}^{n}: d\left(u, A_{\varphi}\right) \leq \delta_{\varphi}+\nu_{\varphi}\right\} \cap B_{\varphi} .
$$

Consequentemente, $\left[\left(\tilde{A_{\varphi}}\right)_{\varphi}\right] \subseteq A$.

Seja $u \in A$. Então por definição de conjunto interno $\exists p_{1} \in \mathbb{N}, \forall \varphi \in \mathcal{A}_{p_{1}}(n)$, e $\left(u_{\varphi}\right)_{\varphi}$ representante com $u_{\varphi} \in A_{\varphi}$. Além disso, podemos fazer seja $u \in A$ ainda por definição de conjunto interno $\exists p_{2} \in \mathbb{N}, \forall \varphi \in \mathcal{A}_{p_{2}}(n)$, e $u_{\varphi} \in A_{\varphi}$, com $d\left(u_{\varphi}, A_{\varphi}\right) \leq \delta_{\varphi}+\nu_{\varphi}$. Assim, tomando o $p=\max \left\{p_{1}, p_{2}\right\}$ e $\forall \varphi \in \mathcal{A}_{p}(n)$, temos $u_{\varphi} \in A_{\varphi}$ e $u_{\varphi} \in \tilde{A_{\varphi}}$, portanto $A \subseteq\left[\left(\tilde{A}_{\varphi}\right)_{\varphi}\right]$.

Consequentemente, $\sup _{u_{\varphi} \in \tilde{A}_{\varphi}}\left|u_{\varphi}\right| \leq \sup _{u_{\varphi} \in \tilde{A}_{\varphi}} \inf _{a \in A_{\varphi}}\left|u_{\varphi}-a\right|+|a| \leq \delta_{\varphi}+\nu_{\varphi}+\alpha_{-r}^{\bullet}(\varphi)$. Portanto, $\sup _{u \in\left[\left(\tilde{A}_{\varphi}\right)_{\varphi}\right]} \leq \delta+\alpha_{-r}^{\bullet}$ temos que $\left(\tilde{A}_{\varphi}\right)_{\varphi}$ é uma rede $A$ - $J$ limitada.

Vamos agora provar um resultado ao qual considero um dos mais importantes dessa seção. 
Proposição 3.1.23. Seja $A \cap B$ conjunto interno e que tem representantes $A$-J limitados. Então $\left[\left(A_{\varphi} \cap B_{\varphi}\right)_{\varphi}\right]=A \cap B$.

Demonstração. Sejam $A \cap B \subseteq A$ e $\left(C_{\varphi}\right)_{\varphi}$ um representante de $A \cap B$. Assim, por definição de conjunto interno, temos que,

Seja $u \in A \cap B, \exists p_{1} \in \mathbb{N}, \forall \varphi \in \mathcal{A}_{p_{1}}(n), e \exists\left(u_{\varphi}\right)_{\varphi} \operatorname{com} u_{\varphi} \in C_{\varphi}$ e segue que $\operatorname{dist}(u, A)=0$. Agora por definição de nulidade temos,

$\exists p_{2} \in \mathbb{N}, \exists \gamma_{2} \in \Gamma, \forall \varphi \in \mathcal{A}_{p_{2}}(n), \exists C_{2}>0, \eta_{2}>0, \forall \varepsilon<\eta_{2}$, temos

$$
\delta^{A}=\sup _{u_{\varphi_{\varepsilon}} \in C_{\varphi_{\varepsilon}}} d\left(u_{\varphi_{\varepsilon}}, A_{\varphi_{\varepsilon}}\right) \leq C \varepsilon^{\gamma_{2}(q)-p_{2}}, \forall q \geq p_{2} .
$$

Como $A \cap B \subseteq B$ e consideramos $\left(C_{\varphi}\right)_{\varphi}$ um representante de $A \cap B$, e como anteriormente temos que, seja $u \in A \cap B, \exists p_{1} \in \mathbb{N}, \forall \varphi \in \mathcal{A}_{p_{1}}(n), e \exists\left(u_{\varphi}\right)_{\varphi} \operatorname{com} u_{\varphi} \in C_{\varphi}$. Então $\operatorname{dist}(u, B)=$ 0 , agora por definição de nulidade temos

$\exists p_{3} \in \mathbb{N}, \exists \gamma_{3} \in \Gamma, \forall \varphi \in \mathcal{A}_{p_{3}}(n), \exists C_{3}>0, \eta_{3}>0, \forall \varepsilon<\eta_{3}$, temos por definição de nula

$$
\delta^{B}=\sup _{u_{\varphi_{\varepsilon}} \in C_{\varphi_{\varepsilon}}} d\left(u_{\varphi_{\varepsilon}}, B_{\varphi_{\varepsilon}}\right) \leq C \varepsilon^{\gamma_{3}(q)-p_{3}}, \forall q \geq p_{3} .
$$

Sejam $p=\max \left\{p_{1}, p_{2}, p_{3}\right\}, C=\max \left\{C_{2}, C_{3}\right\}$ e $\gamma=\max \left\{\gamma_{2}, \gamma_{3}\right\}$ e $\eta=\min \left\{\eta_{2}, \eta_{3}\right\}$ tal que $\forall \varphi \in \mathcal{A}_{p}(n)$, temos que $u_{\varphi} \in C_{\varphi}$ e $\delta^{A} \leq C \varepsilon^{\gamma(q)-p}$ e $\delta^{B} \leq C \varepsilon^{\gamma(q)-p}, \forall q \geq p$.

Agora podemos escolher uma rede nula $\left(\nu_{\varphi}\right)_{\varphi} \in \mathbb{R}^{\mathcal{A}_{0}}$ e $\nu_{\varphi}>0 \mathrm{com}$

$$
\begin{aligned}
& \tilde{A}_{\varphi}=\left\{u \in \mathbb{R}^{n} \mid d\left(u, A_{\varphi}\right) \leq \delta_{\varphi}^{A}+\nu_{\varphi}\right\} \\
& \tilde{B}_{\varphi}=\left\{u \in \mathbb{R}^{n} \mid d\left(u, B_{\varphi}\right) \leq \delta_{\varphi}^{B}+\nu_{\varphi}\right\}
\end{aligned}
$$

e por $(3.2)$ e (3.3), temos $\left(\sup _{u \in \tilde{A}_{\varphi}} d\left(u, A_{\varphi}\right)\right)_{\varphi} \in \mathcal{N}$. Da mesma forma $\left(\sup _{u \in A_{\varphi}} d\left(u, \tilde{A}_{\varphi}\right)\right)_{\varphi}$ $\in \mathcal{N}$. Consequentemente $\left(d_{H}\left(\tilde{A}_{\varphi}, A_{\varphi}\right)\right)_{\varphi} \in \mathcal{N}$ e segue também que $\left(d_{H}\left(\tilde{B}_{\varphi}, B_{\varphi}\right)\right)_{\varphi} \in \mathcal{N}$. Portanto $\left(\tilde{A}_{\varphi}\right)_{\varphi}$ é um representante de $A$, e $\left(\tilde{B}_{\varphi}\right)_{\varphi}$ é representante de $B$. Temos que $\left[\left(\tilde{A}_{\varphi} \cap\right.\right.$ $\left.\left.\tilde{B}_{\varphi}\right)_{\varphi}\right] \subseteq A \cap B$.

Seja $u \in A \cap B$ e $\log 0 \exists p \in \mathbb{N}, \forall \varphi \in \mathcal{A}_{p}(n), \exists\left(u_{\varphi}\right)_{\varphi}$ com $u_{\varphi} \in C_{\varphi}$, e escolhendo uma rede nula $\left(\nu_{\varphi}\right)_{\varphi}$ podemos ter $d\left(u_{\varphi}, A_{\varphi}\right) \leq \delta^{A}+\nu_{\varphi}$ e $d\left(u_{\varphi}, B_{\varphi}\right) \leq \delta^{B}+\nu_{\varphi}$. Portanto dessa forma temos $A \cap B \subseteq\left[\left(\tilde{A}_{\varphi} \cap \tilde{B}_{\varphi}\right)_{\varphi}\right]$.

Teorema 3.1.24 (Princípio da saturação). Sejam $\left(A_{n}\right)_{n \in \mathbb{N}}$ uma sequência de conjuntos internos de $\tilde{\Omega}_{f}$, com a propriedade de interseção finita. Suponhamos também que exista ponto positivo $t=t(n) \in \mathbb{R}$ tal que $[u]_{2} \leq \alpha_{-t}^{\bullet}, \forall u \in A_{1} \cap \ldots \cap A_{n}$ e $\forall n \in \mathbb{N}$. Então $\cap_{n \in \mathbb{N}} A_{n} \neq \emptyset$.

Demonstração. Escolhamos $u_{n} \in A_{1} \cap \ldots \cap A_{n}$, então existe $\left(u_{n, \varphi}\right)_{\varphi}$ representante de $u_{n}$ e $A=\left[\left(A_{n, \varphi}\right)_{\varphi}\right]$ então pela Proposição 3.1.6 temos que $d\left(u_{n, \varphi}, A_{j, \varphi}\right)_{\varphi} \in \mathcal{N}, \forall j \leq n$ e pelo fato $p_{j} \in \mathbb{N}, \exists \gamma_{j} \in \Gamma, \forall \varphi \in \mathcal{A}_{p_{j}}(n) \forall j \leq n$, e $\forall q \geq p_{j}, \exists C>0, \exists \eta>0$ tal que $\forall \varepsilon<\eta$, temos que , $\exists\left(\varepsilon_{q, k}\right)_{k \in \mathbb{N}} \operatorname{com} \lim _{k \rightarrow \infty} \varepsilon_{q, k}=0$, tal que $d\left(u_{n, \varphi_{\varepsilon}}, A_{j, \varphi_{\varepsilon}}\right) \leq C \varepsilon^{\gamma(q)-p_{j}}$.

Dessa forma definamos $v_{\varphi}:=u_{n, \varphi}, \forall \varphi \in \mathcal{A}_{p_{n}}(n) \backslash \mathcal{A}_{p_{n+1}}(n)$ e sabemos que $d\left(v_{\varphi_{\varepsilon}}, A_{j, \varphi_{\varepsilon}}\right) \leq$ $C \varepsilon^{\gamma(q)-p_{j}}, \forall \varepsilon_{q, k+1}<\varepsilon \leq \varepsilon_{q, k}, \forall q, k \in \mathbb{N}$, por outro lado, sabemos também que por hipótese temos $\left[u_{n}\right]_{2} \leq \alpha_{-t}^{\bullet}$ e pelo Lema $3.1 .13 \alpha_{-t}^{\bullet} \leq \alpha_{-t-1}^{\bullet}$, como $v=\left[\left(v_{\varphi}\right)_{\varphi}\right]$ satisfaz $[v]_{2} \leq$ $\alpha_{-t-1}^{\bullet}$, então $v \in \tilde{\Omega}_{f}$. Como acontece pela Proposição $3.1 .6 d\left(v_{\varphi}, A_{j, \varphi}\right)_{\varphi} \in \mathcal{N}, \forall j \leq n$, pela proposição 3.1.6. Logo $v_{\varphi} \in A_{j, \varphi}, \forall j \in \mathbb{N}$ e $\forall \varphi \in \mathcal{A}_{p_{n}}(n) \backslash \mathcal{A}_{p_{n+1}}(n)$ e portanto $v \in$ $\cap_{n \in \mathbb{N}} A_{n}$.

\section{Proposição 3.1.25.}

(a) Sejam E,F espaços normados e $A=\left[\left(A_{\varphi}\right)_{\varphi}\right] \subseteq \mathcal{G}_{E}, B=\left[\left(B_{\varphi}\right)_{\varphi}\right] \subseteq \mathcal{G}_{F}$, conjuntos internos. Então $A \times B=\left[\left(A_{\varphi} \times B_{\varphi}\right)_{\varphi}\right]$ é um subconjunto interno de $\mathcal{G}_{E} \times \mathcal{G}_{F}$. 
(b) Seja $A=\left[\left(A_{\varphi}\right)_{\varphi}\right]$ um subconjunto interno de $\mathcal{G}_{E} \times \mathcal{G}_{F}$. Então a projeção $\operatorname{pr}_{\mathcal{G}_{E}}(A)$ de $A$ sobre $\mathcal{G}_{E}$ é um subconjunto de $\left[\operatorname{pr}_{E}\left(A_{\varphi}\right)_{\varphi}\right]$. Se $A$ tem um representante $A$-J limitado na topologia natural, então $\operatorname{pr}_{\mathcal{G}_{E}}(A)=\left[\left(\operatorname{pr}_{E}\left(A_{\varphi}\right)\right)_{\varphi}\right]$ é interno.

Demonstração. (a) A topologia de $E \times F$ é gerada por $\|(u, v)\|=\max \left\{\|u\|_{E},\|v\|_{F}\right\}$. Sejam $I D_{E}: E \rightarrow E$ e $I D_{F}: F \rightarrow F$ a identidade de $E$, respectivamente de $F$ e $I D: E \times F \rightarrow$ $E \times F$ por $I D=I D_{E} \times I D_{F}$. Então definimos $T_{E}: \mathcal{G}_{E} \rightarrow \mathcal{G}_{E}$ e $T_{F}: \mathcal{G}_{F} \rightarrow \mathcal{G}_{F}$ por $T_{E}(A):=\left[\left(I D_{E}\left(A_{\varphi}\right)\right)_{\varphi}\right]$, e $T_{F}(A):=\left[\left(I D_{F}\left(A_{\varphi}\right)\right)_{\varphi}\right]$. Consequentemente definimos o seguinte objeto,

$$
T: \mathcal{G}_{E} \times \mathcal{G}_{F} \rightarrow \mathcal{G}_{E \times F} \text { por } T(A \times B):=\left[\left(I D\left(A_{\varphi} \times B_{\varphi}\right)\right)_{\varphi}\right] .
$$

Mostramos que $T$ é homomorfismo. Iremos provar agora que $T$ é um isomorfismo. De fato, seja $\left[\left(A_{\varphi} \times B_{\varphi}\right)_{\varphi}\right] \in \mathcal{G}_{E \times F}$. Então $\left[\left(A_{\varphi} \times B_{\varphi}\right)_{\varphi}\right]=\left[\left(I D\left(A_{\varphi} \times B_{\varphi}\right)\right)_{\varphi}\right]$ e por definição $T(A \times B)=$ $\left[\left(I D\left(A_{\varphi} \times B_{\varphi}\right)\right)_{\varphi}\right]$ onde $A=\left[\left(A_{\varphi}\right)_{\varphi}\right]$ e $B=\left[\left(B_{\varphi}\right)_{\varphi}\right]$. Assim, $T$ é sobrejetivo.

Agora para $A \times B \in \operatorname{Ker}(T)$, temos $T(A \times B)=\left[\left(I D\left(A_{\varphi} \times B_{\varphi}\right)\right)_{\varphi}\right] \in \mathcal{N}_{E \times F}$, segue que por definição de conjunto interno que $\exists p_{1} \in \mathbb{N}$ tal $u \in\left[\left(I D\left(A_{\varphi} \times B_{\varphi}\right)\right)_{\varphi}\right]$. Assim, $\forall \varphi \in \mathcal{A}_{p_{1}}$ temos $u_{\varphi} \in A_{\varphi} \times B_{\varphi}$, e segue $u_{\varphi}=u_{1, \varphi} \times u_{2, \varphi}$ e por definição de nulidade,

$\exists p_{2} \in \mathbb{N}, \exists \gamma \in \Gamma ; \forall q \geq p_{2}, \forall \varphi \in \mathcal{A}_{q}, \exists C>0, \exists \eta>0 ; \forall \varepsilon<\eta$ temos que $\forall u \in$ $\left[\left(\left(A_{\varphi} \times B_{\varphi}\right)\right)_{\varphi}\right] \operatorname{com}\left\|u_{\varphi_{\varepsilon}}\right\| \leq C \varepsilon^{\gamma(q)-p_{2}}$ tomamos $p=\max \left\{p_{1}, p_{2}\right\}$ então $\forall \varphi \in \mathcal{A}_{p}$ temos $\left\|u_{\varphi_{\varepsilon}}\right\| \leq C \varepsilon^{\gamma(q)-p}$ consequemtemente temos $\left\|u_{\varphi_{\varepsilon}}\right\|=\max \left\{\left\|u_{1, \varphi_{\varepsilon}}\right\|,\left\|u_{2, \varphi_{\varepsilon}}\right\|\right\} \leq C \varepsilon^{\gamma(q)-p}$, o que implica que $\left[\left(A_{\varphi}\right)_{\varphi}\right] \in \mathcal{N}_{E}$ e $\left[\left(B_{\varphi}\right)_{\varphi}\right] \in \mathcal{N}_{F}$. Logo $T$ é isomorfismo.

(b) Tome $u \in \operatorname{pr}_{\mathcal{G}_{E}}(A)$ existe algum $v \in \mathcal{G}_{F}$ tal que $(u, v) \in A$, então por definição de conjunto interno, existe $p \in \mathbb{N} ; \forall \varphi \in \mathcal{A}_{p}$ existem representantes $\left(u_{\varphi}, v_{\varphi}\right)$ de $(u, v)$ com $\left(u_{\varphi}, v_{\varphi}\right) \in A_{\varphi}$ e temos ainda $u_{\varphi} \in \operatorname{pr}_{E} A_{\varphi}$, portanto $u \in\left[\left(p r_{E}\left(A_{\varphi}\right)\right)_{\varphi}\right]$.

Reciprocamente, seja $u \in\left[\left(\operatorname{pr}_{E}\left(A_{\varphi}\right)\right)_{\varphi}\right]$, por definição de conjunto interno existe $p \in \mathbb{N}$; $\forall \varphi \in \mathcal{A}_{p}$, existe um representante $\left(u_{\varphi}\right)_{\varphi}$ de com $u \in \operatorname{pr}_{E}\left(A_{\varphi}\right)$. Nesta condições existe algum representante $\left(v_{\varphi}\right)_{\varphi}$ de $v$ com $v_{\varphi} \in F$, tal que $\left(u_{\varphi}, v_{\varphi}\right) \in A_{\varphi}, \forall \varphi \in \mathcal{A}_{p}$. Como $\left(A_{\varphi}\right)_{\varphi}$ é uma rede $A$ - $J$ limitada, então $\left(v_{\varphi}\right)_{\varphi}$ é uma rede moderada, portanto $v \in \mathcal{G}_{F}$, consequentemente temos $(u, v) \in A$ e $\operatorname{pr}_{\mathcal{G}_{E}}(u, v)=u, \log 0 \operatorname{pr}_{\mathcal{G}_{E}} A=\left[\left(p r_{E}\left(A_{\varphi}\right)\right)_{\varphi}\right]$.

\subsection{Funções Internas}

Vamos apresentar mais um conceito que nos permite descrever quando um conjunto é interno através de uma aplicação. Começamos então definindo o conceito de funções internas. Estendemos alguns resultados encontrados em [OV08].

Uma aplicação $f: A \subseteq \tilde{\mathbb{R}}^{d} \rightarrow \tilde{\mathbb{R}}^{d^{\prime}}$ é chamada interna se o seu gráfico graf $(f)=$ $[\{(x, f(x)): x \in A\}]$ é um subconjunto interno de $\tilde{\mathbb{R}}^{d+d^{\prime}}$. Da mesma forma $f: A \subseteq \tilde{\mathbb{R}}^{d} \rightarrow \tilde{\mathbb{C}}$ é chamada interna se o graf $(f)$ é um subconjunto interno de $\tilde{\mathbb{R}}^{d+2}$.

Para construir nosso resultado principal desse capítulo precisamos enunciar alguns resultados, e começamos pelo seguinte lema.

Lema 3.2.1. Seja $f: A \subseteq \tilde{\mathbb{R}}^{d} \rightarrow \tilde{\mathbb{R}}^{d^{\prime}}$ uma aplicação interna. Se $A$ e $f(A)$ forem $A-J$ limitados na topologia natural, então $A$ e $f(A)$ são conjuntos internos.

Demonstração. De fato, é só aplicar a Proposição 3.1.25, pois $A$ e $f(A)$ são projeções do gráfico de $f$ limitados na topologia natural.

Sabemos que para cada $h \in \mathcal{G}_{f}(\Omega)$ induz uma aplicação $h: \tilde{\Omega}_{c} \rightarrow \tilde{\mathbb{C}}$ por meios da ação $\left[\left(x_{\varphi}\right)_{\varphi}\right] \rightarrow\left[\left(h_{\varphi}\left(x_{\varphi}\right)\right)_{\varphi}\right]$ (que é independente dos representantes) e temos o seguinte resultado. 
Lema 3.2.2. Seja $h \in \mathcal{G}_{f}(\Omega)$. Então para cada $A \subset \tilde{\Omega}_{c}$, a aplicação $h: A \rightarrow \tilde{\mathbb{C}}$ é interna. Além disso, $A$ e $h(A)$ são limitados na topologia natural.

Demonstração. O gráfico da aplicação induzida é o conjunto $\left[\left(\left\{\left(x, h_{\varphi}(x)\right): x \in A_{\varphi}\right\}\right)_{\varphi}\right]$ (que é definido independentemente dos representantes $\left(h_{\varphi}\right)_{\varphi}$ de $\left.h\right)$. Vejamos que $A$ é limitado na topologia natural por definição de $\tilde{\Omega}_{c}$ e $h(A)$ é limitado na topologia natural pois as redes $\left(h_{\varphi}\right)_{\varphi}$ são moderadas.

Vamos agora enunciar nosso resultado principal deste capitulo, e vamos relacionar aplicações internas com conjuntos internos.

Proposição 3.2.3. Seja $f: A \subseteq \tilde{\mathbb{R}}^{d} \rightarrow \tilde{\mathbb{R}}^{d^{\prime}}$ aplicação interna com $A$ e $f(A) A-J$ limitados. Então existe uma rede $\left(f_{\varphi}\right)_{\varphi}$ com $f_{\varphi} \in C^{\infty}\left(\mathbb{R}^{d}, \mathbb{R}^{d^{\prime}}\right)$ tal que para cada $\tilde{x} \in A$ com representante $\left(x_{\varphi}\right)_{\varphi}, e\left(f_{\varphi}\left(x_{\varphi}\right)\right)_{\varphi}$ é representante de $f(\tilde{x})$. Além disso, $f$ é uniformemente contínua(na topologia natural)sobre $A$.

Demonstração. Seja $\left[\left(R_{\varphi}\right)_{\varphi}\right]$ o gráfico de $f$ (podemos supor que $R_{\varphi}$ é fechado e consequentemente compacto). Então para,

$u \in\left[\left(R_{\varphi}\right)_{\varphi}\right], \exists p_{1} \in \mathbb{N}$, tal que $\forall \varphi \in \mathcal{A}_{p_{1}}$ existem representantes que $\left(u_{\varphi}\right)_{\varphi} \operatorname{com} u_{\varphi} \in R_{\varphi}$, e por $A$ e $f(A)$ serem limitados $\exists M \in \mathbb{N}, \exists \eta_{1}>0 ; \forall \varepsilon<\eta_{1}$, temos $R_{\varphi_{\varepsilon}} \subset B\left(0, \iota(\varphi)^{-M} \varepsilon^{-M}\right)$.

Notamos que $\forall \varphi \in \mathcal{A}_{0}$ podemos construir uma função $g_{\varphi}: \mathbb{R}^{d} \rightarrow \mathbb{R}^{d^{\prime}}$, por compacidade existe $\left(x_{\varphi}, y_{\varphi}\right) \in R_{\varphi}$ tal que $x \in \mathbb{R}^{d}$ com $\left|x_{\varphi}-x\right|$ é ínfimo. Assim, $g_{\varphi}(x):=y_{\varphi}($ não nescessariamente único).

Sejam $\tilde{x} \in A$ com representante $\left(x_{\varphi}\right)_{\varphi}$ e $\tilde{y}=f(\tilde{x})$ com representante $\left(y_{\varphi}\right)_{\varphi}$. Então, desde que $(\tilde{x}, \tilde{y}) \in\left[\left(R_{\varphi}\right)_{\varphi}\right]$, existe $\left(x_{\varphi}^{\prime}, y_{\varphi}^{\prime}\right) \in R_{\varphi} \operatorname{com}\left|x_{\varphi}-x_{\varphi}^{\prime}\right|_{\varphi}$ e $\left|y_{\varphi}-y_{\varphi}^{\prime}\right|_{\varphi}$ são nulos. Vamos mostrar que $\left(g_{\varphi}\right)_{\varphi}$ não dependem dos representantes. Para outro representante $\left(x_{\varphi}^{\prime \prime}\right)_{\varphi}$ de $\left(x_{\varphi}\right)_{\varphi}$, então $\left|x_{\varphi}^{\prime \prime}-x_{\varphi}^{\prime}\right| \leq\left|x_{\varphi}^{\prime \prime}-x_{\varphi}\right|+\left|x_{\varphi}-x_{\varphi}^{\prime}\right|$ e temos que,

existe $p_{2} \in \mathbb{N}, \exists \gamma_{2} \in \Gamma, \forall \varphi \in \mathcal{A}_{p_{2}}, \exists C_{2}>0, \exists \eta_{2}>0 ; \forall \varepsilon<\eta_{2}$, com

$$
\left|x_{\varphi_{\varepsilon}}-x_{\varphi_{\varepsilon}}^{\prime}\right| \leq C_{2} \varepsilon^{\gamma_{2}(q)-p_{2}}, \forall q \geq p_{2} \text {. }
$$

existe $p_{3} \in \mathbb{N}, \exists \gamma_{3} \in \Gamma, \forall \varphi \in \mathcal{A}_{p_{3}}, \exists C_{3}>0, \exists \eta_{3}>0 ; \forall \varepsilon<\eta_{3}$, com

$\left|x_{\varphi_{\varepsilon}}-x_{\varphi_{\varepsilon}}^{\prime \prime}\right| \leq C_{3} \varepsilon^{\gamma_{3}(q)-p_{3}}, \forall q \geq p_{3}$.

Consideramos,

(*) $p=\max \left\{p_{1}, p_{2}, p_{3}\right\}$ e $\eta=\min \left\{\eta_{1}, \eta_{2}, \eta_{3}\right\}$ e $\gamma=\max \left\{\gamma_{2}, \gamma_{3}\right\} \operatorname{com} C=\max \left\{C_{2}, C_{3}\right\}$.

É facil ver que, $\left|x_{\varphi}^{\prime \prime}-x_{\varphi}^{\prime}\right| \leq C \varepsilon^{\gamma(q)-p}, \forall q \geq p$ e em particular podemos ter, $\mid x_{\varphi}^{\prime \prime}-$ $x_{\varphi}|\leq| x_{\varphi}^{\prime}-x_{\varphi} \mid$, com a mesma ordem. Desta maneira $\left(x_{\varphi}^{\prime \prime}, g_{\varphi}\left(x_{\varphi}\right)\right)_{\varphi} \in R_{\varphi}$, em particular $d\left(\left(x_{\varphi}^{\prime \prime}, g_{\varphi}\left(x_{\varphi}\right)\right), R_{\varphi}\right)_{\varphi}$ é nula. Portanto $\left(g_{\varphi}\left(x_{\varphi}\right)\right)_{\varphi}$ é um representante de $f(\tilde{x})$.

Vamos mostrar que $\left(g_{\varphi}\right)_{\varphi}$ é uniformemente contínuo sobre $A$, ou seja, $\left(g_{\varphi}\right)_{\varphi}$ é um representante de $f$, independentemente dos pontos de $A$.

Nas condições de $(*)$ suponhamos que $\forall q \geq p$ existe $\varphi \in \mathcal{A}_{q}$ e $\forall \varepsilon<\eta$, temos que existe $\left(\varepsilon_{m}\right)_{m \in \mathbb{N}} \operatorname{com} \lim _{m \rightarrow \infty} \varepsilon_{m}=0$, e $x_{\varphi_{\varepsilon_{m}}} \in A_{\varphi_{\varepsilon_{m}}} \operatorname{com} x_{\varphi_{\varepsilon_{m}}}^{\prime} \in \mathbb{R}^{d}$ tal que $\left(A_{\varphi}\right)_{\varphi}$ é um representante $A-J$ limitado satisfazendo,

$\forall m \in \mathbb{N}$ existe $n \in \mathbb{N}\left|x_{\varphi_{\varepsilon_{m}}}-x_{\varphi_{\varepsilon_{m}}}^{\prime}\right| \leq \iota(\varphi)^{m} \varepsilon^{m} \mathrm{e}\left|g_{\varphi_{\varepsilon_{m}}}\left(x_{\varphi_{\varepsilon_{m}}}\right)-g_{\varphi_{\varepsilon_{m}}}\left(x_{\varphi_{\varepsilon_{m}}}^{\prime}\right)\right| \geq \iota(\varphi)^{n} \varepsilon^{n}$.

Nas condições do Lema 1.2.32, temos que existem $\left(q_{l}\right)_{l \in \mathbb{N}}$ e $\left(a_{l}\right)_{l \in \mathbb{N}}$ tal que $\varphi_{q_{l}} \in \mathcal{A}_{a_{l}}$ e $\left(\varepsilon_{q_{l}, k}\right)_{k \in \mathbb{N}} \operatorname{com} \lim _{k \rightarrow \infty} \varepsilon_{q_{l}, k}=0$. Então existe $s\left(\varphi_{q_{l}, \varepsilon_{q_{l}, k}}\right):=x_{q_{l}, \varepsilon_{q_{l}, k}}$ e $\forall \varepsilon_{q_{l}, k} \notin\left\{\varepsilon_{m}: m \in \mathbb{N}\right\}$, e $\forall l, k \in \mathbb{N}$, definimos $s\left(\varphi_{\varepsilon_{m}}\right):=x_{\varphi_{\varepsilon_{m}}}^{\prime}$. Agora é só mostrar que $s=\left[(s(\varphi))_{\varphi}\right]$ é uma rede moderada e suportado compactamente. 
Como $s\left(\varphi_{q_{l}, \varepsilon_{q_{l}, k}}\right) \in B\left(0, \iota(\varphi)^{-M} \varepsilon_{q_{l}, k}^{-M}\right)$, então podemos tomar um $K \subset B\left(0, \iota(\varphi)^{-M} \varepsilon_{q_{l}, k}^{-M}\right)$ compacto tal que $s\left(\varphi_{q_{l}, \varepsilon_{q_{l}, k}}\right) \in K$. Podemos tomar o fecho da bola por exemplo.

Como $A$ é $A-J$ limitado na topologia natural, temos que nas condições de $(*)$, para todo $\tilde{x} \in A$ acontece, $\left|x_{\varphi_{\varepsilon}}\right| \leq C^{\prime} \varepsilon^{-M}$ para alguma constante $C^{\prime}$. Desta maneira,

$$
\exists l_{0}>0 \text { tal que } a_{l_{0}} \geq p \text { e } \forall l \geq l_{0} \text {, obtemos que }
$$

$\exists \varphi_{q_{l}} \in \mathcal{A}_{a_{l}}$ com $a_{l} \geq a_{l_{0}}, \exists \varepsilon^{\prime}$ tal que para $\varphi \in \mathcal{A}_{a_{l}}$, acontece que $\varphi_{\varepsilon}=\varphi_{q_{l}, \varepsilon \varepsilon^{\prime}}=\varphi_{q_{l}, \varepsilon_{q_{l}, k}}$ para $\operatorname{algum} k \in \mathbb{N}$.

Então $\forall \varepsilon<\eta$, temos

$$
\begin{aligned}
\left|s\left(\varphi_{\varepsilon}\right)\right|=\left|s\left(\varphi_{q_{l}, \varepsilon \varepsilon^{\prime}}\right)\right| & =\left|s\left(\varphi_{q_{l}, \varepsilon_{q_{l}, k}}\right)\right| \leq \iota\left(\varphi_{q_{l}}\right)^{-M} \varepsilon_{q_{l}, k}^{-M} \leq \iota\left(\varphi_{q_{l}}\right)^{-M}\left(\varepsilon \varepsilon^{\prime}\right)^{-M}, \text { e tomamos } \\
C^{\prime} & =\iota\left(\varphi_{q_{l}}\right)^{-M} \varepsilon^{\prime} \text { obtemos que }\left|s\left(\varphi_{\varepsilon}\right)\right| \leq C^{\prime} \varepsilon^{-M},
\end{aligned}
$$

consequentemente $s=\left[(s(\varphi))_{\varphi}\right]$ é uma rede moderada. Quando $s\left(\varphi_{\varepsilon_{m}}\right)=x_{\varphi_{\varepsilon_{m}}}^{\prime}$ e pelo fato $x_{\varphi_{\varepsilon_{m}}}$ é moderada então $x_{\varphi_{\varepsilon_{m}}}^{\prime}$ é moderado também. Segue que,

$$
\left|x_{\varphi_{\varepsilon_{m}}}-s\left(\varphi_{\varepsilon_{m}}\right)\right| \leq \iota(\varphi)^{m} \varepsilon_{m}^{m} \text { e }\left|x_{\varphi_{q_{l}, \varepsilon_{q}, k}}-s\left(\varphi_{q_{l}, \varepsilon_{q_{l}, k}}\right)\right| \leq \iota\left(\varphi_{q_{l}}\right)^{m} \varepsilon_{q_{l}, k}^{m}, \forall \varphi_{q_{l}} \in \mathcal{A}_{a_{l}} / \mathcal{A}_{a_{l+1}} .
$$

Pelo fato que $\left(x_{\varphi}\right)_{\varphi}$ e $(s(\varphi))_{\varphi}$, poderiam representar o mesmo elemento de $A$, e sua imagem por $f$ poderiam ser diferentes, temos uma contradição.

Sejam $m \in \mathbb{N}$ e $\varepsilon \in I_{\eta}$, com $\varepsilon \in\left(\frac{1}{m}, \frac{1}{m-1}\right]$ e $\forall \varphi \in \mathcal{A}_{p}$, temos $h_{\varphi} \in C^{0}\left(\mathbb{R}^{d}, \mathbb{R}^{d^{\prime}}\right)$ por uma interpolação linear em cima dos valores de $\left\{g_{\varphi}(x): x \in \mathbb{Z}^{d} / m^{m}\right\}$ (por meios de triangulação de $\left.\mathbb{Z}^{d} / m^{m}\right)$.

Então para cada $x \in \mathbb{R}^{d}$, existe $x_{i} \in \mathbb{Z}^{d} / m^{m}, i \in I_{x}$ finito(números de elementos de $I_{x}$ dependendo apenas de $d$ )tal que $\left|x-x_{i}\right| \leq D \varepsilon^{m}(D \in \mathbb{R}$ depende apenas do diâmetro $d$ do $d$ - cubo)e para cada $i,\left|h_{\varphi}(x)-g_{\varphi}(x)\right| \leq \max _{j, k \in I_{x}}\left|g_{\varphi\left(x_{j}\right)}-g_{\varphi\left(x_{k}\right)}\right|$.

Agora sejam $\tilde{x} \in A$ com representante $\left(x_{\varphi}\right)_{\varphi}$ e $n \in \mathbb{N}$ a continuidade uniforme diz que existe $m \in \mathbb{N}$ tal que para todo $x, x^{\prime} \in \mathbb{R}^{d}$,

$$
\max \left(\left|x-x_{\varphi_{\varepsilon}}\right|,\left|x^{\prime}-x_{\varphi_{\varepsilon}}\right|\right) \leq \iota(\varphi)^{m} \varepsilon^{m} \Rightarrow\left|g_{\varphi_{\varepsilon}}(x)-g_{\varphi_{\varepsilon}}\left(x^{\prime}\right)\right| \leq \iota(\varphi)^{n} \varepsilon^{n},
$$

e segue que $\left|h_{\varphi_{\varepsilon}}\left(x_{\varphi_{\varepsilon}}\right)-g_{\varphi_{\varepsilon}}\left(x_{\varphi_{\varepsilon}}\right)\right| \leq\left|h_{\varphi_{\varepsilon}}\left(x_{\varphi_{\varepsilon}}\right)-g_{\varphi_{\varepsilon}}\left(x_{i, \varphi_{\varepsilon}}\right)\right|+\left|g_{\varphi_{\varepsilon}}\left(x_{i, \varphi_{\varepsilon}}\right)-g_{\varphi_{\varepsilon}}\left(x_{\varphi_{\varepsilon}}\right)\right| \leq$ $2 \iota(\varphi)^{n} \varepsilon^{n}$ (para algum $\left.x_{i, \varphi_{\varepsilon}} \in I_{x_{\varphi_{\varepsilon}}}\right)$.

Logo, $\left(h_{\varphi}\left(x_{\varphi}\right)\right)_{\varphi}$ é também representante de $f(\tilde{x})$. Finalmente pelo Teorema StoneWeierstrass garante a existência de $f_{\varphi} \in C^{\infty}\left(\mathbb{R}^{d}, \mathbb{R}^{d^{\prime}}\right)$ tal que $\sup _{|x|<\iota(\varphi)^{-m} \varepsilon^{-m}}\left|f_{\varphi_{\varepsilon}}(x)-h_{\varphi_{\varepsilon}}(x)\right| \leq$ $\iota(\varphi)^{n} \varepsilon^{n}$ para $\varepsilon \leq \frac{1}{m}$. Portanto $\left(f_{\varphi}\right)_{\varphi}$ é representantes de $f$ é independente dos pontos de $A$.

\subsection{Aplicações}

Vamos fazer diversas aplicações para o Teorema 3.1.24, então começamos enunciando a seguinte proposição.

Proposição 3.3.1. Existe um elemento $U=\left[\left(u_{\psi}\right)_{\psi}\right] \in \mathcal{G}_{f}\left(\mathbb{R}^{d}\right)$ tal que

$$
\begin{gathered}
\int U(x) d x=1, \int x^{\beta} U(x) d x=0 \text { para todo, }|\beta| \geq 1 \text {, supp } U \subseteq\left\{x \in \mathbb{R}^{d}:|x| \leq\right. \\
1\}, \text { mais precisamente, supp } u_{\psi} \subseteq\left\{x \in \mathbb{R}^{d}:|x| \leq 1\right\} \text { para todo } u_{\psi} .
\end{gathered}
$$

Demonstração. Vamos aplicar o Teorema 3.1.24 para o conjunto $E=C^{\infty}\left(\mathbb{R}^{d}\right)$, começamos construindo os conjuntos $(n \geq 0)$

$$
\mathcal{A}_{n}=\left\{\varphi \in \mathcal{D}\left(\mathbb{R}^{d}\right) \mid \int \varphi(x) d x=1, \int x^{\beta} \varphi(x) d x=0 \text { para todo } 1 \leq|\beta| \leq n, \text { supp } \varphi \subseteq\{x \in\right.
$$$$
\left.\left.\mathbb{R}^{d}:|x| \leq 1\right\}\right\} \text {. }
$$ 
Sabemos que esse conjuntos $\mathcal{A}_{n}$ são diferentes do vazio. Agora escolhemos $\varphi_{n} \in \mathcal{A}_{n}$ e $\iota\left(\varphi_{n}\right)=$ $t_{n}$ e $\psi \in \mathcal{A}_{p_{n}} \backslash \mathcal{A}_{p_{n+1}}$ de forma que $\frac{1}{\iota(\psi)} \geq t_{n}$. Definimos os conjuntos $(\forall n \geq 0)$

$$
A_{n, \psi}=\left\{\varphi \in \mathcal{A}_{n} \mid\|\varphi\| \leq \frac{1}{\iota(\psi)}\right\}
$$

Os conjuntos $A_{n, \psi} \neq \emptyset$. De fato, pois se $\varphi \in \mathcal{A}_{n}$ e $\varepsilon>0$ e $\varphi_{\varepsilon} \in \mathcal{A}_{n}$, então para algum $n \in \mathbb{N}$ e $\varepsilon>0$, temos que acontece $\left\|\varphi_{n, \varepsilon}\right\| \leq \frac{1}{\iota\left(\psi_{\varepsilon}\right)}$.

Vejamos que para cada $\psi$ fixado ocorre $A_{n+1, \psi} \subseteq A_{n, \psi}$. Conseguimos assim que $A_{n+1}=$ $\left[\left(A_{n+1, \psi}\right)_{\psi}\right] \subseteq A_{n}=\left[\left(A_{n, \psi}\right)_{\psi}\right]$. Então os conjuntos internos $A_{n}$ satisfazem as condições do teorema da saturação, e obtemos que $\cap_{n \in \mathbb{N}} A_{n} \neq \emptyset$, ou seja, existe $U \in \mathcal{G}_{f}(\Omega)$ tal que $U$ satisfaz as condições do Teorema 3.1.24.

Existe um representante $\left(u_{\psi}\right)_{\psi}$ de $U$ tal que $u_{\psi} \in A_{n, \psi}$ de modo que, para todo $\beta$, e $|\beta| \geq 1$, existe um $n \in \mathbb{N}$ tal que $u_{\psi} \in \mathcal{A}_{n}$ com $\int u_{\psi}(x) d x=1, \int x^{\beta} u_{\psi}(x) d x=0$, ou seja, $\int U(x) d x=1, \int x^{\beta} U(x) d x=0$ assim, concluimos o resultado.

$\mathrm{Na}$ Proposição 3.3.1 podemos encontrar a função generalizada $U$ perto da norma $-L^{1}$, como era esperado. Apresentamos a prova no seguinte resultado.

Proposição 3.3.2. Para cada $\delta>0$ existe um elemento $U \in \mathcal{G}_{f}\left(\mathbb{R}^{d}\right)$ tal que a Propriedade da Proposição 3.3 .1 acontece, e em adição $\int|U(x)| d x \leq 1+\delta$.

Demonstração. A prova é na mesma linha da anterior, vamos considerar $d=1$. Seja o conjunto

$$
\begin{gathered}
A_{0}^{\prime}(\delta)=\left\{\varphi \in A_{0, \psi}\left|\int\right| \varphi(x) \mid d x \leq 1+\delta\right\} . \text { Então } A_{0}^{\prime}(\delta) \text { é diferente do vazio, pois tomamos } \\
\text { uma função } \varphi \text { não negativa, e supp } \varphi \subset\{x \in \mathbb{R} \| x \mid \leq 1\} \text { com } \int \varphi(x) d x=1 .
\end{gathered}
$$

Agora suponhamos por indução que o resultado é verdade para $A_{n-1}^{\prime}(\delta / 2)$. Consequentemente vamos tomar $\varphi_{1} \in A_{n-1}^{\prime}(\delta / 2)$ com constantes $a, b$ reais e $0<\eta<1$ escolhidos, e definamos $\varphi(x)=a \varphi_{1}(x)+b \varphi_{1}(x / \eta)$. Notemos que $\varphi$ satisfaz

$$
\begin{gathered}
\int \varphi(x) d x=a+b \eta, \int x^{k} \varphi(x) d x=0 \text { para } 1 \leq k \leq n-1 \text { e } \int x^{n} \varphi(x) d x= \\
\left(a+b \eta^{n+1}\right) \int x^{n} \varphi_{1}(x) d x .
\end{gathered}
$$

Assim resolvendo o sistema $a+b \eta=1, a+b \eta^{n+1}=0$, obtemos $a=\frac{-\eta^{n}}{1-\eta n}<0, b=$ $\frac{1}{\eta-\eta^{n+1}}>0$. Logo,

$$
\int|\varphi(x)| d x \leq(|a|+|b| \eta) \int\left|\varphi_{1}(x)\right| d x \leq \frac{1+\eta^{n}}{1-\eta n}\left(1+\frac{\delta}{2}\right) \text { então o resultado segue. }
$$

Tomamos $\varphi \in A_{n+1}^{\prime}(\delta)$ então $\varphi \in \mathcal{A}_{n+1}$ e consequentemente $\varphi \in \mathcal{A}_{n} \operatorname{com} \int|\varphi(x)| d x \leq$ $1+\delta$ e segue que satisfaz $\varphi \in A_{n}^{\prime}(\delta)$. Notamos que $\forall n \in \mathbb{N}$ acontece $A_{n+1}^{\prime}(\delta) \subseteq A_{n}^{\prime}(\delta)$ e temos a cadeia encaixante de conjuntos internos $M_{n+1}^{\prime}=\left[A_{n+1}^{\prime}(\delta)\right] \subseteq M_{n}^{\prime}=\left[A_{n}^{\prime}(\delta)\right]$. Portanto pelo princípio da saturação temos $\cap_{n \in \mathbb{N}} M_{n}^{\prime} \neq \emptyset$.

Outra aplicação que vamos dar do princípio da saturação é a completude da esfera de $\tilde{\mathbb{R}}_{f}$ e $\tilde{\mathbb{C}}$. Uma prova foi dada para a álgebra de Colombeau simplificada em [OV08].

Proposição 3.3.3. Seja $\tilde{\mathbb{K}}$ sendo $\tilde{\mathbb{R}}_{f}$ ou $\tilde{\mathbb{C}}_{f}$. Seja $\left(B_{n}\right)_{n \in \mathbb{N}}$ uma cadeia decrescente de bolas sharp $B_{n}=\left\{x \in \tilde{\mathbb{K}}:\left\|x-a_{n}\right\|_{e} \leq r_{n}\right\}$, onde $a_{n} \in \tilde{\mathbb{K}}, r_{n} \in \mathbb{R}$ e $r_{n}>0$. Então $\cap_{n \in \mathbb{N}} B_{n} \neq \emptyset$.

Demonstração. Vamos supor que $\left(B_{n}\right)_{n \in \mathbb{N}}$ é estritamente decrescente. Nesta situação provaremos o teorema. Como $a_{n+1} \in B_{n}$ e $B_{n+1} \subset B_{n}$, então $\alpha_{-\operatorname{logr}_{n+1}} \leq \alpha_{-\operatorname{logr}_{n}} \operatorname{com}\left(r_{n}\right)_{n}$ decrescente a zero. Seja $\left(a_{n+1, \varphi}\right)_{\varphi}$ representante de $a_{n+1}$ e construímos

$$
V_{n, \varphi}=\left\{x \in \mathbb{K}|| x-a_{n+1, \varphi} \mid \leq \iota(\varphi)^{-\log r_{n}}\right\}
$$


Então temos o conjunto interno $V_{n}=\left[\left(V_{n, \varphi}\right)_{\varphi}\right]$. Seja $x \in B_{n+1}$, logo $\left\|x-a_{n+1}\right\|_{e} \leq r_{n+1}$ o que implica $e^{-V\left(\hat{x}-\hat{a}_{n+1}\right)}<r_{n+1}$, consequentemente $V\left(\hat{x}-\hat{a}_{n+1}\right)>-\log r_{n+1}$. Logo $\exists p \in$ $\mathbb{N} ; \forall \varphi \in \mathcal{A}_{p}, \exists \eta>0 ; \forall \varepsilon<\eta$, temos

$$
\begin{aligned}
& \lim _{\varepsilon \rightarrow 0} \iota(\varphi)^{\log r_{n+1}} \varepsilon^{\log r_{n+1}}\left|\hat{x}_{\varphi_{\varepsilon}}-\hat{a}_{n+1, \varphi_{\varepsilon}}\right|=0 \text { e } \forall \varepsilon<\eta \text { obtemos, } \\
& \left|\hat{x}_{\varphi_{\varepsilon}}-\hat{a}_{n+1, \varphi_{\varepsilon}}\right| \leq \hat{\alpha}^{\bullet}\left(\varphi_{\varepsilon}\right)^{-\log r_{n+1}} \leq \hat{\alpha}^{\bullet}\left(\varphi_{\varepsilon}\right)^{-\log r_{n}}
\end{aligned}
$$

logo concluímos que $x \in V_{n}$.

Agora seja $y \in V_{n}$. Então $\exists p \in \mathbb{N}$ tal que $\forall \varphi \in \mathcal{A}_{p}$. desta maneira existe $\left(y_{\varphi}\right)_{\varphi}$ representante de $y \operatorname{com} y_{\varphi} \in V_{n, \varphi}$, e obtemos $\left|y_{\varphi}-a_{n+1, \varphi}\right| \leq \iota(\varphi)^{-\log r_{n}}$. Por outro lado, $\exists \eta>0$ tal que $\forall \varepsilon<\eta$, temos $\iota(\varphi)^{\log r_{n}} \varepsilon^{\log r_{n}}\left|\hat{y}_{\varphi_{\varepsilon}}-\hat{a}_{n+1, \varphi_{\varepsilon}}\right|<1$, fazendo $\varepsilon \rightarrow 0$, temos

$$
\iota(\varphi)^{\log r_{n}} \lim _{\varepsilon \rightarrow 0} \varepsilon^{-\log r_{n}}\left|\hat{y}_{\varphi_{\varepsilon}}-\hat{a}_{n+1, \varphi_{\varepsilon}}\right|=0
$$

Portanto, $-\log r_{n} \in V\left(\hat{y}-\hat{a_{n+1}}\right)$ o que implica $\left\|y-a_{n+1}\right\|_{e}<e^{-V\left(\hat{y}-a_{n+1}\right)} \leq e^{\log r_{n}} \leq r_{n}$, logo $y \in B_{n}$. Então $B_{n+1} \subseteq V_{n} \subseteq B_{n}$ segue pelo princípio da saturação que $\cap B_{n}=\cap V_{n} \neq \emptyset$.

$O$ próximo resultado que vamos apresentar também através do princípio da saturação, está relacionado com a completude da álgebra plena de Colombeau. Definimos os seguinte conjunto $V(U, r)=\left\{V \in \mathcal{G}_{f} \mid\|U-V\|_{\beta, m} \leq \alpha_{r}^{\bullet}\right\}$, vamos usar esses conjuntos no próximo resultado.

Proposição 3.3.4. Seja $\mathcal{G}_{f}(\Omega)$ com a topologia natural de Colombeau. Então $\mathcal{G}_{f}(\Omega)$ é um espaço completo.

Demonstração. Seja uma sequência $\left(f_{j}\right)_{j \in \mathbb{N}}$ de Cauchy em $\mathcal{G}_{f}(\Omega)$ e consideramos uma sequência de números positivos $\left(r_{n}\right)_{n \in \mathbb{N}} \searrow_{0}$.

Como $\left(f_{j}\right)_{j \in \mathbb{N}}$ é uma sequência de cauchy, então:

$\forall r_{n} \in \mathbb{R}_{+}^{*}, \exists p \in \mathbb{N} ; \forall \beta \in \mathbb{N}^{m}, \operatorname{com} \forall p \geq|\beta|$ e $l \in \mathbb{N}, \exists n_{0} \in \mathbb{N} ; \forall n \geq n_{0}, \exists j_{n}$ tal que $\forall t^{\prime}, t \geq j_{n}$, temos $\left\|f_{t^{\prime}}-f_{t}\right\|_{\beta, l} \leq \alpha_{r_{n}}^{\bullet}$.

Sabemos que $B_{n+1} \subset B_{n}$. Assim vamos aproximar as bolas sharp com conjuntos internos, para isso definimos o conjunto interno $A_{n}$ por $A_{n}=V_{n}\left(f_{j_{n}} ;-\log r_{n}\right)$. Agora seja, $h \in$ $B_{n+1}\left(f_{j_{n+1}} ; r_{n+1}\right)$. Então $\left\|h-f_{j_{n+1}}\right\|=e^{-V\left(\hat{h}-\hat{f} j_{n+1}\right)} \leq r_{n+1}$ implica que $V\left(\hat{h}-\hat{f} j_{n+1}\right) \geq$ $-\log r_{n+1}$, que por consequência temos $\left\|\partial^{\beta}\left(\hat{h}\left(\varphi_{\varepsilon}, \bullet\right)-\hat{f}_{j_{n+1}}\left(\varphi_{\varepsilon}, \bullet\right)\right)\right\|_{l} \leq \varepsilon^{-\log r_{n+1}}$. Quando tomamos a classe $\left\|h-f_{j_{n+1}}\right\|_{\beta, m} \leq \alpha_{-\log r_{n+1}}^{\bullet} \leq \alpha_{-\log r_{n}}$, segue que $h \in A_{n}$.

Por outro lado seja $h \in A_{n}$ e como $\left(f_{j}\right)_{j \in \mathbb{N}}$ é sequência de Cauchy, vamos usar o seguinte resultado:

$\forall \nu \in \mathbb{N}^{*}$, para toda sequência finita $s=\left(m_{k}, p_{k}\right)_{1 \leq k \leq \nu}$ em $\mathbb{N}^{2}$ e $\left.\forall r_{n} \in\right] 0,\left[1, \exists j_{n} \in \mathbb{N}\right.$ tal que existem $\left.\left.r, j \geq j_{n}, \varepsilon_{j_{n} r} \in I=\right] 0,1\right], N_{k} \in \mathbb{N}$ tal que se $0<\varepsilon<\varepsilon_{j_{n} r}$ e $1 \leq k \leq \nu$, então

$$
\begin{aligned}
\left\|\partial^{\beta}\left(\hat{f}_{r}-\hat{f}_{j_{n+1}}\right)\left(\left(\varphi_{\varepsilon}, \bullet\right)\right)\right\|_{m_{k}} \leq & \varepsilon^{-\log r_{n}}, \forall \varphi \in \mathcal{A}_{N_{K}}, \forall|\beta| \leq p_{k} . \text { Então verifica-se } \\
& \left\|f_{j_{n+1}}-f_{r}\right\|_{e}<r_{n}
\end{aligned}
$$

então faça $\hat{f}_{r}=\hat{h}$ e $\operatorname{logo}$ obtemos $h \in B_{n}\left(f_{j_{n+1}} ; r_{n}\right)$. Sabemos que $\forall f_{j_{n+1}} \in B_{n}\left(f_{j_{n}}, r_{n}\right)$ temos $B_{n}\left(f_{j_{n+1}}, r_{n}\right)=B_{n}\left(f_{j_{n}}, r_{n}\right)$, e concluímos que $h \in B_{n}\left(f_{j_{n}}, r_{n}\right)$. Portanto temos que $B\left(f_{j_{n+1}}, r_{n+1}\right) \subseteq V_{n} \subseteq B\left(f_{j_{n}}, r_{n}\right)$, agora pelo princípio da saturação $\cap_{n \in \mathbb{N}} B_{n}=\cap_{n \in \mathbb{N}} V_{n} \neq$ $\emptyset$.

Definição 3.3.5. Seja $x \in \tilde{\mathbb{R}}_{f}$ chamamos $x$ de infinitamente grande se $|x| \geq y$ para todo $y \in \tilde{\mathbb{R}}_{f, c}$.

Então denotamos o conjunto de números infinitamente grande por $\tilde{\mathbb{R}}_{f, \infty}$, semelhantemente como em [Ver11]. 
Lema 3.3.6. Se $x \in \tilde{\mathbb{R}}_{f}$ é infinitamente grande, então $|x| \geq n, \forall n \in \mathbb{N}$.

Demonstração. Seja $x \in \tilde{\mathbb{R}}_{f, \infty}$ então $|x| \geq y \in \tilde{\mathbb{R}}_{f, c}$, e existe um representante $\left(y_{\varphi}\right)_{\varphi}$ de $y$, $\exists K \subset \subset \Omega, \exists p_{1} \in \mathbb{N}$ e $\forall \varepsilon \in I_{\eta}, \forall \varphi \in \mathcal{A}_{p_{1}}$ tal que $y_{\varphi_{\varepsilon}} \in K$. Agora construa o conjunto $\Omega:=\cup_{n=1}^{\infty}\left[n, n+\frac{1}{2}\right]$. Dado $\left(\varepsilon_{q, k}\right)_{k \in \mathbb{N}} \searrow_{0}$, para cada $\varphi \in \mathcal{A}_{q}$ e $x_{q, k} \in\left[n, n+\frac{1}{2}\right]$, tomamos pelo Lema 1.2.32 que existe uma aplicação $s_{n}: \mathcal{A}_{0} \rightarrow \Omega$ tal que $s_{n}\left(\varphi_{q_{l}, \varepsilon_{q_{l}, k}}\right)=x_{q_{l}, k}, \forall l, k \in$ $\mathbb{N}, \forall \varphi_{q_{l}} \in \mathcal{A}_{a_{l}} / \mathcal{A}_{a_{l+1}}$.

Como $(*) \tilde{\Omega}=\Omega_{M} / \sim$. Então existe $N \in \mathbb{N}, \forall \varphi \in \mathcal{A}_{N}, \exists C>0, \exists \eta>0$, e $\forall \varepsilon \in I_{\eta}$, temos que para $u \in \tilde{\Omega}$, acontece que $\left|u_{\varphi_{\varepsilon}}\right| \leq C \varepsilon^{-N}$.

Por outro lado, como temos que $\exists l_{0}$ tal que $q_{l} \geq N, \forall l \geq l_{0}$ e $\forall \varphi_{q_{l}} \in \mathcal{A}_{a_{l}} / \mathcal{A}_{a_{l+1}}$, então $s_{n}\left(\varphi_{q_{l}, \varepsilon_{q_{l}, k}}\right) \in\left[n, n+\frac{1}{2}\right] \forall n \in \mathbb{N}$ e em particular é limitado também satisfazendo $(*)$. Segue que que é suportado compactamente. Assim, $s_{n} \in \tilde{\mathbb{R}}_{f, c}$, e temos que $|x| \geq s_{n}$; em particular $\forall \varphi \in \mathcal{A}_{a_{l}} / \mathcal{A}_{a_{l+1}}$, e dado $\varepsilon>0 \exists \varepsilon^{\prime} \operatorname{com}\left|x_{\varphi_{\varepsilon}}\right| \geq s_{n}\left(\varphi_{\varepsilon}\right)=s_{n}\left(\varphi_{q_{l}, \varepsilon \varepsilon^{\prime}}\right)=s_{n}\left(\varphi_{q_{l}, \varepsilon_{q_{l}, k}}\right)$ para algum $k \in \mathbb{N}$, obtemos que $\left|x_{\varphi_{\varepsilon}}\right| \geq n, \forall l, k, n \in \mathbb{N}$.

Proposição 3.3.7. Overspill e Underspill Seja $A=\left[\left(A_{\varphi}\right)_{\varphi}\right] \subseteq \tilde{\mathbb{R}}_{f}$ um conjunto interno. Então A contém arbitrários grandes números suportados compactamente se, e somente se, A contém arbitrários números $a \in A$ menor ou igual a números infinitamente grandes. Ou seja, $\forall x \in \tilde{\mathbb{R}}_{f, c}$, existe é um $a \in A \cap \tilde{\mathbb{R}}_{f, c}$ tal que $|a| \geq|x|$ se, e somente se, para todo $x \in \tilde{\mathbb{R}}_{f, \infty}$ existe um $a \in A \cap \tilde{\mathbb{R}}_{f, \infty}$ tal que $|a| \leq|x|$.

Demonstração. $(\Rightarrow)$ seja $\tilde{x} \in \tilde{\mathbb{R}}_{f, \infty}$ com representante $\left(x_{\varphi}\right)_{\varphi}$. Então, para cada $n \in \mathbb{N}$ definimos o conjunto $\hat{B}_{n}=\left\{y \in A_{\varphi}: n \leq|y| \leq\left|x_{\varphi}\right|\right\}$, que pelo Lema 3.3.6 é diferente do vazio $(\hat{B} \neq \emptyset)$, assim, $B_{n}=\left[\left(\left\{y \in A_{\varphi}: n \leq|y| \leq\left|x_{\varphi}\right|\right\}\right)_{\varphi}\right] \neq \emptyset$, e temos que $B_{n}$ é limitado na topologia natural. De fato,

seja $y \in B_{n}$ e então $\left(y_{\varphi}\right)_{\varphi}$ representante de $y$ e por definição de conjunto interno $\exists p_{1} \in$ $\mathbb{N} ; \forall \varphi \in \mathcal{A}_{p_{1}}$, temos $y_{\varphi} \in \hat{B}_{n}$ e também por definição de moderada existe $N \in \mathbb{N} ; \forall \varphi \in \mathcal{A}_{N}$ e $\exists C>0, \exists \eta>0, \forall \varepsilon \in I_{\eta} \operatorname{com}\left|x_{\varphi_{\varepsilon}}\right| \leq C \varepsilon^{-N}$.

Agora tomamos $p=\max \left\{p_{1}, N\right\}$ e temos $n \leq\left|y_{\varphi_{\varepsilon}}\right| \leq\left|x_{\varphi_{\varepsilon}}\right| \leq C \varepsilon^{-p}$ e podemos fazer $\iota(\varphi)^{-p}:=C$ e $p:=r$. Conforme a Definição 3.1 .8 temos $\left|y_{\varphi_{\varepsilon}}\right| \leq\left|x_{\varphi_{\varepsilon}}\right| \leq \iota(\varphi)^{-r} \varepsilon^{-r} \Rightarrow|y| \leq$ $|x| \leq \alpha_{-r}^{\bullet}, \operatorname{logo} B_{n}$ é $A$ - $J$ limitado na topologia natural $\forall n \in \mathbb{N}$. Em particular, temos:

$\left\{\tilde{y} \in A \cap \tilde{\mathbb{R}}_{f, c}:|\tilde{y}| \geq n+1\right\} \subseteq B_{n} \subseteq\{\tilde{y} \in A: n \leq|\tilde{y}| \leq|\tilde{x}|\}$

$\operatorname{Logo},\left(B_{n}\right)_{n \in \mathbb{N}}$ é uma sequência decrescente de subconjuntos internos de $\tilde{\mathbb{R}}_{f}$ limitada na topologia natural e não-vazio. Pelo príncipio da saturação, existe $z \in \cap_{n \in \mathbb{N}} B_{n}$, e segue que $z \in A \cap \tilde{\mathbb{R}}_{f, \infty}$ e $|z| \leq|\tilde{x}|$.

$(\Leftarrow) \forall \tilde{x} \in \tilde{\mathbb{R}}_{f, c}$ existe $a \in A \cap \tilde{\mathbb{R}}_{f, c}$ tal que $|a| \geq \tilde{x}$. Consideramos $\left(x_{\varphi}\right)_{\varphi}$ representante de $\tilde{x}$ e definamos o conjunto

$\hat{B}=\left\{y \in A_{\varphi}:|y| \geq\left|x_{\varphi}\right|\right\}$. Dessa maneira temos o conjunto interno $B=\left[\left(\left\{y \in A_{\varphi}:\right.\right.\right.$ $\left.\left.\left.|y| \geq\left|x_{\varphi}\right|\right\}\right)_{\varphi}\right]$. Assim, por definição $A \cap \tilde{\mathbb{R}}_{f, \infty} \subseteq B \subseteq A \cap\left\{\tilde{y} \in \tilde{\mathbb{R}}_{f}:|\tilde{y}| \geq|\tilde{x}|\right\}$. Pela Proposição 3.1.16 existe $b \in B$ tal que $|b|$ é mínimo e por hipótese, $|b| \leq|\tilde{y}|$, para cada $\tilde{y} \in \tilde{\mathbb{R}}_{f, \infty}$.

Por hipótese, $|b| \leq|\tilde{y}|$ para cada $\tilde{y} \in \tilde{\mathbb{R}}_{f, \infty}$. Então $|b| \leq N$ para algum $N \in \mathbb{N}$. Se não for o caso, suponhamos o contrário, poderíamos achar um representante $\left(b_{\varphi}\right)_{\varphi}$ de $b$, tal que $\forall n \in \mathbb{N}, \exists p_{1} \in \mathbb{N}$ e $\forall \varphi \in \mathcal{A}_{p_{1}}$ temos $b_{\varphi} \in \hat{B}$ e além disso $\exists \eta>0 ; \forall 0<\varepsilon_{n}<\eta \operatorname{com}\left|b_{\varphi_{\varepsilon_{n}}}\right| \geq n$, poderíamos achar $\forall q \geq p_{1}, \exists \varphi \in \mathcal{A}_{q}, \exists\left(\varepsilon_{q, k}\right)_{k \in \mathbb{N}} \searrow_{0}$, consequentemente definimos,

$s\left(\varphi_{q, \varepsilon_{n}}\right)=\frac{n}{2}$, para cada $n \in \mathbb{N}$ e $s\left(\varphi_{q, \varepsilon_{q, k}}\right)=\iota(\varphi)^{-1} \varepsilon_{q, k}^{-1}$, caso contrário $\left(\varepsilon_{q, k} \notin\left\{\varepsilon_{n} \mid n \in\right.\right.$ $\mathbb{N}\}$ ), então teríamos uma rede $s(\varphi)_{\varphi}$ com algum representante $s \in \tilde{\mathbb{R}}_{f, \infty}$ com $|b| \nless s$ uma contradição. Logo $b \in A \cap \tilde{\mathbb{R}}_{f, c}$ e portanto $|b| \geq|\tilde{x}|$.

Proposição 3.3.8. Seja $A \subseteq \tilde{\mathbb{R}}_{f}$ conjunto interno. Se $A$ contém todos os números infinitamente grande maior, ou igual, do que $C \in \tilde{\mathbb{R}}_{f, c}$ em valor absoluto. Então A contém todos números infinitamente grande menor em valor absoluto do que algum $R \in \tilde{\mathbb{R}}_{f, \infty}$. 
Demonstração. Sejam $A=\left[\left(A_{\varphi}\right)_{\varphi}\right]$ e $C$ como na afirmação, $N \in \mathbb{N}$, e $|C| \leq N$. Então cada $n \in \mathbb{N}$, escolha um $l \in \mathbb{N}$ e definamos o conjunto:

$$
\begin{gathered}
\hat{B}_{n, \varphi_{q_{l}}}=\left\{x \in \mathbb{R}: n \leq|x| \leq \iota\left(\varphi_{q_{l}}\right)^{-1} \text { e } N \leq|y| \leq|x| \operatorname{com} d\left(y, A_{\varphi_{q_{l}}}\right) \leq \iota\left(\varphi_{q_{l}}\right)^{n}\right\} \text { escolha } \\
\varphi_{q_{l}} \in \mathcal{A}_{a_{l}} / \mathcal{A}_{a_{l+1}}
\end{gathered}
$$

e obtemos temos o conjunto interno $B_{n}=\left[\left(\hat{B}_{n, \varphi_{q_{l}}}\right)_{\varphi_{q_{l}}}\right] \forall l \in \mathbb{N}$. Seja $u \in B_{n+1}$. Então para $\left(u_{\varphi_{q_{l}}}\right)_{\varphi_{q_{l}}}$ representante de $u$, segue por definição de conjunto interno que $\exists p \in \mathbb{N}$ tal que $\forall \varphi_{q_{l}} \in \mathcal{A}_{p} \operatorname{com} u_{n, \varphi_{q_{l}}} \in B_{n+1, \varphi_{q_{l}}}$ o que implica $n+1 \leq\left|u_{\varphi_{q_{l}}}\right| \leq \iota\left(\varphi_{q_{l}}\right)^{-1}$ e $N \leq|y| \leq\left|u_{\varphi_{q_{l}}}\right|$ com $d\left(y, A_{\varphi_{q_{l}}}\right) \leq \iota\left(\varphi_{q_{l}}\right)^{n+1}$. Em particular tempos, $n \leq\left|u_{\varphi_{q_{l}}}\right| \leq \iota\left(\varphi_{q_{l}}\right)^{-1}$, segue que $u_{\varphi_{q_{l}}} \in B_{n, \varphi_{q_{l}}}$. Logo, $B_{n+1} \subseteq B_{n}, \forall n \in \mathbb{N}$.

Por outro lado, $\forall u \in B_{n}$ obtemos que $\left(u_{\varphi}\right)_{\varphi} \operatorname{com}|u| \leq \alpha_{-1}^{\bullet}$ e $d\left(u_{\varphi}, A_{\varphi}\right)_{\varphi} \in \mathcal{N}$, dessa maneira, $B_{n}$ é limitado na topologia natural $\forall n \in \mathbb{N}$.

Também para cada $n \in \mathbb{N}$ o conjunto é $B_{n} \neq \emptyset$. Supondo o contrário, teríamos que existe $\left(l_{m}\right)_{m \in \mathbb{N}}$ tal que, $B_{n, \varphi_{l_{m}}}=\emptyset$, então $\forall p \in \mathbb{N}, \forall \gamma \in \Gamma ; \exists q \geq p e \exists \varphi \in \mathcal{A}_{q}, \forall C>0, \forall \eta>$ 0 com $\exists 0<\varepsilon_{0}<\eta$ tal que satisfazem

$$
d\left(y_{\varphi_{q_{l_{m}}, \varepsilon_{0}}}, A_{\varphi_{q_{l_{m}}, \varepsilon_{0}}}\right) \geq C \varepsilon_{0}^{\gamma(q)-p}
$$

podemos escolher uma sequência de $C_{n}>0$ e $\left(\varepsilon_{m}\right)_{m \in \mathbb{N}} \operatorname{com} \lim _{m \rightarrow \infty} \varepsilon_{m}=0$ tal que $0<$ $\varepsilon_{m}<\eta$ e $y_{\varphi_{\varepsilon_{m}}} \in \mathbb{R} \mathrm{com}$,

(*) $N \leq\left|y_{\varphi_{q_{l_{m}}, \varepsilon_{m}}}\right| \leq n$ de forma que acontece $d\left(y_{\varphi_{q_{l_{m}}, \varepsilon_{m}}}, A_{\varphi_{q_{l_{m}}, \varepsilon_{m}}}\right) \geq C \varepsilon_{m}^{\gamma(q)-p}, \forall m \in \mathbb{N}$.

Então definimos o conjunto $T_{1}\left(\varphi_{q_{l_{m}}}\right)=\left\{\varepsilon_{m} \mid\left(\varphi_{q_{l_{m}}}\right)_{\varepsilon_{m}}\right.$ que satisfaz $\left.(*)\right\}$ e $T_{2}=\left\{\varphi_{q_{l_{m}, \varepsilon_{m}}} \mid \varepsilon_{m}\right.$ $\left.\in T_{1}\right\}$, então $T_{1}\left(\varphi_{q_{l_{m}}}\right) \in \mathcal{S}$, segue que $T_{2} \in S_{f}$. Logo para cada $\varphi \in T_{2}^{c}$ existe $\left(\varepsilon_{q, k}\right)_{k \in \mathbb{N}} \searrow_{0}$ e para $\varepsilon_{q, k} \notin T_{1}(\varphi)$ defina $y_{\varphi_{\varepsilon_{q, k}}}:=N$.

Então temos pelo Lema 1.2.32 $\left(y_{\varphi_{q_{l_{m}}}}\right)_{\varphi_{q_{l_{m}}}}$ é uma rede moderada com $\tilde{y}=\left[\left(y_{\varphi_{q_{l_{m}}}}\right)_{\varphi_{q_{l_{m}}}}\right] \in$ $\tilde{\mathbb{R}}_{f, c} \operatorname{com}|\tilde{y}| \geq N$. Mas por hipótese, isto poderia implicar que $\tilde{y} \in A$ e pela Proposição 3.1.6, temos $d\left(y_{\varphi,}, A_{\varphi}\right)_{\varphi} \in \mathcal{N}$.

Veja que $d=\left[\left(d\left(y_{\varphi}, A_{\varphi}\right)\right)_{\varphi}\right] \in \overline{\mathbb{R}}_{f}$. Por outro lado usando a relação de ordem de $\overline{\mathbb{R}}_{f}$ consulte [AJOS08b], temos que $0<\chi_{T_{2}} d \leq d$, então $d \in \overline{\mathbb{R}}_{f}^{*}$, por isso $\tilde{y} \notin A$, uma contradição da hipótese, $\operatorname{logo} B_{n} \neq \emptyset$.

Pelo princípio da saturação Teorema 3.1.24 existe $z \in \cap_{n \in \mathbb{N}} B_{n}$. Em particular $\forall n \in$ $\mathbb{N}, \exists l \in \mathbb{N}, \exists p \in \mathbb{N}, \exists \gamma \in \Gamma$ e para $\forall q \geq p, \forall \varphi \in \mathcal{A}_{q}, \exists C>0, \exists \eta>0, \exists\left(\varepsilon_{q, k}\right)_{k \in \mathbb{N}} \searrow_{0}$ com $0<\varepsilon_{q, k} \leq \eta$ tal que $N \leq\left|y_{\varphi_{q_{m}}, \varepsilon_{q, k}}\right| \leq\left|z_{\varphi_{q_{l_{m}}, \varepsilon_{q, k}}}\right|-1$ implica que $d\left(y, A_{\varphi_{q_{l_{m}}, \varepsilon}}\right) \leq C \varepsilon^{\gamma(q)-p}$, $\forall \varepsilon_{q, k+1}<\varepsilon \leq \varepsilon_{q, k}$. Logo pela Proposição 3.1.6 $\tilde{y} \in A$ para todo $\tilde{y} \in \overline{\mathbb{R}}_{f}$ com $N \leq|\tilde{y}| \leq$ $|z|-1$. 


\section{Capítulo 4}

\section{Zeros de Funções Holomorfas Generalizados}

Consideramos $\mathbb{K}=\mathbb{C}, \Omega \subset \mathbb{C}$ aberto não vazio, $\mathcal{H}(\Omega)=\left\{f \in C^{1}(\Omega ; \mathbb{C}): \bar{\partial}(f)=0\right\}$ e $\mathcal{H G}(\Omega)=\left\{f \in \mathcal{G}_{f}(\Omega): \bar{\partial}(f)=0\right\}$. É obvio que $\overline{\mathbb{C}}=\overline{\mathbb{R}}+i \overline{\mathbb{R}}$, onde $i^{2}=-1$ tem-se que $\overline{\mathbb{C}} e ́$ $\overline{\mathbb{R}}$ - isomorfo $a \overline{\mathbb{R}}^{2}$. Se $z=x+i y$, com $x, y \in \overline{\mathbb{R}}$, considere os operadores:

$$
\frac{\partial}{\partial z}:=\frac{1}{2}\left\{\partial_{x}-i \partial_{y}\right\}, \frac{\partial}{\partial \bar{z}}:=\frac{1}{2}\left\{\partial_{x}+i \partial_{y}\right\}
$$

Dado $f \in \mathcal{H}(\Omega)$ denotemos por $\mathcal{R}_{f}$ o conjunto de zeros da $f$ em $\Omega$. Sabemos que $\mathcal{R}_{f} \cap \Omega=$ $\emptyset$, a não ser que $f=0$. Isto é conhecido como o Teorema da Identidade para funções holomorfas. A mesma coisa não acontece para um elemento $f \in \mathcal{H} \mathcal{G}(\Omega)$. De fato, considere $f(z)=\chi_{A} z, z \in \overline{\mathbb{C}}$, onde $A \subset \mathcal{A}_{0}$ é um subconjunto cuja função característica não é nula, e seja $z_{n}=\chi_{A^{c}} \alpha_{n}$. Por exemplo, no caso da álgebra simplificada, pode-se tomar $A=\left\{\frac{1}{n}\right.$ : $n \in \mathbb{N}\}$. Então $f\left(z_{n}\right)=0=f(0), \forall n,\left(z_{n}\right)$ converge para 0 em $\overline{\mathbb{C}}$ mas $f$ não é nula em $\mathcal{H G}(\mathbb{C})$. Isto prova que o Teorema da Identidade não é válida em $\mathcal{G}_{f}(\Omega)$.

Temos claramente que $\mathcal{H}(\Omega) \subset \mathcal{H} \mathcal{G}(\Omega)$ e logo, dada $f \in \mathcal{H}(\Omega)$, podemos considerar o seu conjunto de zeros em $\overline{\mathbb{C}}$, denotando isto por $\mathcal{G}_{f} \mathcal{R}_{f}$. Claramente temos que $\mathcal{R}_{f} \subset \mathcal{G}_{f} \mathcal{R}_{f}$. Colombeau mostrou que se $f \in \mathcal{H} \mathcal{G}(\Omega)$ e existir um disco $D \subset \Omega \cap \mathcal{G}_{f} \mathcal{R}_{f}$ então $f=0$. Este resultado foi generalizado por Khelif-Scarpalezos. Neste capitulo vamos responder uma pergunta de Colomebeau no contexto da álgebra full: Se $f \in \mathcal{H}(\Omega)$, qual a relação entre os conjuntos $\mathcal{G}_{f} \mathcal{R}_{f}$ e $R_{f}$ ?

Para ilustrar a nossa abordagem deste problema consideremos $f_{0}(z)=\left(z-z_{0}\right)\left(z-z_{1}\right) \in$ $\mathcal{H}(\mathbb{C}), z_{0} \neq z_{1}$. Tem-se que $\mathcal{R}_{f_{0}}=\left\{z_{0}, z_{1}\right\}$. Seja $e \in \overline{\mathbb{C}}_{f}$ um idempotente e seja $z=e z_{0}+$ $(1-e) z_{1} \in \overline{\mathbb{C}}$. Então, considerando $f_{0}$ como função generalizada, tem-se que $f_{0}(z)=\left(\right.$ e $z_{0}+$ $\left.(1-e) z_{1}-z_{0}\right)\left(e z_{0}+(1-e) z_{1}-z_{1}\right)=(1-e)\left(z_{1}-z_{0}\right) \cdot e\left(z_{0}-z_{1}\right)=-(1-e) \cdot e \cdot\left(z_{0}-z_{1}\right)^{2}=0$. Isto mostra que que $z_{e}=e z_{0}+(1-e) z_{1} \in \mathcal{G}_{f} \mathcal{R}_{f}$.

$O$ argumento acima pode ser generalizado facilmente. Dados $f \in \mathcal{H}(\Omega), z_{1}, \cdots, z_{n}$ elementos distintos de $\mathcal{R}_{f}$ e idempotentes ortogonais $e_{1}, \cdots, e_{n}$ tal que $1=e_{1}+\cdots+e_{n}$. Consideremos os elementos $w_{0}=e_{1} z_{1}+\cdots e_{n} z_{n}$. Podemos escrever $f(z)=\left(z-z_{1}\right) \cdots(z-$ $\left.z_{n}\right) g(z), g(z) \in \mathcal{H}(\Omega)$. Disto segue-se facilmente que $f\left(w_{0}\right)=0$ e logo $w_{0} \in \mathcal{G}_{f} \mathcal{R}_{f}$. Este exemplo estabelece portanto uma ligação entre os conjuntos internos e os zeros de uma função holomorfa generalizada.

Teorema 4.0.1. Seja $f \in \mathcal{H}(\Omega), \Omega \subset \mathbb{C}$. Então $\mathcal{G}_{f} \mathcal{R}_{f}=\operatorname{interl}\left(\mathcal{R}_{f}\right)=\tilde{\mathcal{R}}_{f, c}$.

Demonstração. Seja $z \in \mathcal{G}_{f} \mathcal{R}_{f}$, seja $\left(z_{\varphi}\right)_{\varphi}$ representante de $z$, e agora estudamos os pontos de acumulação de $\left\{z_{\varphi}: \varphi \in \mathcal{A}_{p}\right.$ e $\left.p \in \mathbb{N}\right\}$. Como $z \in \tilde{\Omega}_{c}$ existe $K \subset \subset \Omega, \exists p_{1} \in \mathbb{N}, \forall \in \mathcal{A}_{p_{1}}$, $\exists \eta_{1}>0$, e $\forall \varepsilon<\eta_{1}$, temos que $z_{\varphi_{\varepsilon}} \in K$. 
Por definição de nulidade, $\exists K \subset \subset \Omega, \exists p \in \mathbb{N}, \exists \gamma \in \Gamma, \forall q \geq p, \forall \varphi \in \mathcal{A}_{p}, \exists C>0$, $\exists \eta_{2}>0 ; \forall \varepsilon<\eta_{2}$, temos:

$\left|f\left(z_{\varphi_{\varepsilon}}\right)\right| \leq C \varepsilon^{\gamma(q)-p}$ sabemos que $f: \tilde{\Omega}_{c} \rightarrow \overline{\mathbb{K}}_{f}$, então defina o conjunto, $B:=\{y \in$ $K \mid K$ compacto,

$\exists p \in \mathbb{N}, \exists \varphi \in \mathcal{A}_{p}, \exists \varepsilon_{n} \rightarrow 0$, com $n \rightarrow \infty$, e $\left.z_{\varphi_{\varepsilon_{n}}} \rightarrow y\right\}$, então $B \neq \emptyset$. De fato, supondo que $B=\emptyset$, logo obtemos:

$(*) \forall p \in \mathbb{N}, \forall \varphi \in \mathcal{A}_{p}, \forall\left(\varepsilon_{n}\right)_{n \in \mathbb{N}}$, com $\lim _{n \rightarrow \infty} \varepsilon_{n}=0$ temos $z_{\varphi_{\varepsilon_{n}}} \nrightarrow y$, e $\forall y \in \Omega$.

Consequentemente nas condições de (*), então existe $p_{3} \in \mathbb{N}, \forall \varphi \in \mathcal{A}_{p_{3}} e \exists \eta_{3}>0$ e $\exists r, r^{\prime}>$ $0 ; \forall \varepsilon<\eta_{3}$, temos $\left|z_{\varphi_{\varepsilon}}-y\right|>r^{\prime}$ e por continuidade implica que $\left|f\left(z_{\varphi_{\varepsilon}}\right)-f(y)\right|>r$.

De fato, defina $H(\cdot)=f(\cdot)-f(y)$ e $f^{-1}(f(y))=\left\{z_{\varphi_{\varepsilon}} \in \Omega \mid f\left(z_{\varphi_{\varepsilon}}\right)=f(y)\right.$, ou $\mid f\left(z_{\varphi_{\varepsilon}}\right)$ $\left.f(y) \mid \leq r_{n} \operatorname{com} r_{n} \rightarrow 0, n \rightarrow \infty\right\}$.

Primeiro, segue que $H\left(z_{\varphi_{\varepsilon}}\right)=f\left(z_{\varphi_{\varepsilon}}\right)-f(y)=0$, então $z_{\varphi_{\varepsilon}} \in Z_{f}(H) \operatorname{com} H \in \mathcal{H}(\Omega)$, portanto existem finitos $\left\{w_{i} \in \Omega \mid i \in\{1, \ldots, N\}\right\}$ e por continuidade temos $\lim _{n \rightarrow \infty} H\left(z_{\varphi_{\varepsilon_{n}}}\right)=$ 0 , com $z_{\varphi_{\varepsilon_{n}}} \rightarrow w_{i}$, absurdo.

Agora seja, $\left|f\left(z_{\varphi_{\varepsilon}}\right)-f(y)\right| \leq r_{n} \operatorname{com} r_{n} \rightarrow 0$, e temos $\left|H\left(z_{\varphi_{\varepsilon_{n}}}\right)\right| \leq\left|f\left(z_{\varphi_{\varepsilon_{n}}}\right)-f(y)\right| \rightarrow 0$, $\operatorname{logo} z_{\varphi_{\varepsilon_{n}}}$ são zeros de $H \in \mathcal{H}(\Omega)$ então existe o $\lim _{n \rightarrow \infty} H\left(z_{\varphi_{\varepsilon_{n}}}\right)=H(w), \operatorname{logo}|H(w)| \leq 0$ segue que existem finitos zeros, ou seja, $\left\{w_{i} \in \Omega \mid i \in\{1, \ldots, N\}\right\}$. Portanto temos $z_{\varphi_{\varepsilon_{n}}} \rightarrow w_{i}$, absurdo.

Então usando a definição de nulidades segue que $\left|f\left(z_{\varphi_{\varepsilon}}\right)\right| \leq C \varepsilon^{\gamma(q)-p}$, tomando o limite encontramos $\lim _{\varepsilon \rightarrow 0}\left|f\left(z_{\varphi_{\varepsilon}}\right)\right|=\left|\lim _{\varepsilon \rightarrow 0} f\left(z_{\varphi_{\varepsilon}}\right)\right| \leq C \varepsilon^{\gamma(q)-p}$, perceba que $\forall q \geq p$ temos que existe $\varphi \in \mathcal{A}_{q}$ e $\left(\exists \varepsilon_{n}\right)_{n \in \mathbb{N}}$ tal que $|f(w)|=\left|\lim _{\varepsilon_{n} \rightarrow 0} f\left(z_{\varphi_{\varepsilon_{n}}}\right)\right| \leq C \varepsilon_{n}^{\gamma(q)-p} \rightarrow 0$, logo $|f(w)|=0$ e portanto $w$ é raiz da função $f$ holomorfa, segue que existe finitas raiz, ou seja, existem $\left\{w_{j}: j \in\{1, \ldots, n\}\right\}$. Então seja $r>0$ e seja $B_{r}\left(w_{j}\right)$ duas-a-duas disjunta e seja $A_{j}=$ $\left\{\varphi \in \mathcal{A}_{0} \mid z_{\varphi} \in B_{r}\left(w_{j}\right)\right\}$ e defina, $B_{j}(\varphi)=\left\{\varepsilon_{n} \mid z_{\varphi_{\varepsilon_{n}}}, n \in \mathbb{N}\right.$, com $\left.z_{\varphi_{\varepsilon_{n}}} \in B_{r}\left(w_{j}\right)\right\}$. Sabemos que $B_{j} \in \mathcal{S}$, de fato, se $B_{j} \notin \mathcal{S}$ então supondo que $\left.] 0, \eta\right] \subset B_{j}$ então $z_{\varphi_{\varepsilon}} \rightarrow w_{j}, \forall \varepsilon<\eta$, porém existem $\varepsilon_{n} \rightarrow 0$ com $n \rightarrow \infty$ e $\exists p \in \mathbb{N}, \varphi \in \mathcal{A}_{p} \operatorname{com} z_{\varphi_{\varepsilon}} \rightarrow w_{i}$ e $i \neq j$, logo uma contradição, pois $B_{i} \cap B_{j}=\emptyset$.

Para cada $\varepsilon \in B_{j}(\varphi)$ implica que $z_{\varphi_{\varepsilon}} \rightarrow w_{j}$, logo segue que $\varphi \in A_{j}$. Portanto $A_{j} \in \mathcal{S}_{f}$. De fato, por definição de nulidade, temos existe $p \in \mathbb{N}, \exists \gamma \in \Gamma, \forall q>p$ e $\forall \varphi \in \mathcal{A}_{q}, \exists C>0, \exists \eta>$ $0 ; \forall \varepsilon<\eta$, temos $\left|f\left(z_{\varphi_{\varepsilon}}\right)\right| \leq C \varepsilon^{\gamma(q)-p}$, agora seja $\varepsilon_{n} \in B_{j}(\varphi) \operatorname{logo}\left|f\left(z_{\varphi_{\varepsilon_{n}}}\right)\right| \leq C \varepsilon_{n}^{\gamma(q)-p}$. Por outro lado, $z_{\varphi_{\varepsilon_{n}}} \rightarrow w_{j}$ e portanto para $\varphi \in \mathcal{A}_{q}$ temos que $z_{\varphi_{\varepsilon_{n}}} \in B_{r}\left(w_{j}\right)$. Segue assim que $B_{j}^{c}(\varphi) \in \mathcal{S}$ para cada $\varphi \in \mathcal{A}_{q}$ o que implica $\exists\left(\varepsilon_{\lambda}\right)_{\lambda \in \mathbb{N}}$ e $\varepsilon_{\lambda} \in B_{j}^{c}(\varphi)$ com $\lambda \rightarrow \infty$ e $\varepsilon_{\lambda} \rightarrow 0$ tal que $\varphi_{\varepsilon_{\lambda}} \in A_{j}^{c}$ e $z_{\varphi_{\varepsilon_{\lambda}}} \rightarrow z_{0}$ onde $z_{0}=w_{i}$ para algum $i \neq j$, para $\varphi \in \mathcal{A}_{q}$. Portanto $\varphi_{\varepsilon_{\lambda}} \in A_{i}$ e $\varphi_{\varepsilon_{\lambda}} \in \mathcal{A}_{q} . \operatorname{Logo} A_{j} \cap \mathcal{A}_{q} \neq \emptyset$ e $A_{j}^{c} \cap \mathcal{A}_{q} \neq \emptyset$ para todo $q$, então $A_{j} \in \mathcal{S}_{f}$.

Finalmente, $f(z)=\left(z-w_{j}\right)^{m_{j}} g_{j}(z)$ com $g\left(w_{j}\right) \neq 0$ e temos $\left|z-w_{j}\right|^{m_{j}} \leq C|f(z)| \mathrm{e}$ portanto $\left|z_{\varphi}-w_{j}\right|^{m_{j}} \leq C\left|f\left(z_{\varphi}\right)\right|$ então $\chi_{A_{j}}(\varphi)\left(z_{\varphi}-w_{j}\right)$ é uma sequência nula, logo $w=$ $\sum_{j=1}^{n} \chi_{A_{j}} w_{j} \in \operatorname{interl}\left(\mathcal{R}_{f}\right)$, o que implica $\mathcal{G}_{f} \mathcal{R}_{f} \subset \operatorname{interl}\left(\mathcal{R}_{f}\right)$.

Reciprocamente, seja $z \in \mathcal{R}_{f}$, defina $F(z):=\left[\varphi \rightarrow f_{\varphi}(z)\right]=0 \in \mathcal{N}(\mathbb{K})$. Agora tome $w \in \operatorname{interl}\left(\mathcal{R}_{f}\right)$ então existem $z_{j} \in \mathcal{R}_{f} \operatorname{com} j=\{1, \ldots, n\}$, tal que temos $w=\sum_{j=1}^{n} \chi_{A_{j}} z_{j}$ defina $\left(w_{\varphi}\right)_{\varphi}=(w)_{\varphi}$, agora tome uma partição $A_{1}, \ldots, A_{n}$ de $\mathcal{S}_{f}$ então faça $\left[\varphi \rightarrow(w)_{\varphi}\right] \subset \tilde{\Omega}_{c}$. Então temos que $F(w)=\left[\varphi \rightarrow f_{\varphi}\left(w_{\varphi}\right)=f(w)=f\left(z_{j}\right)\right]$ para algum $j \in\{1, \ldots, n\}$. Logo $F(w) \in \mathcal{N}(\mathbb{K})$ implica interl $\left(\mathcal{R}_{f}\right) \subseteq \mathcal{G}_{f} \mathcal{R}_{f}$. 


\section{Capítulo 5}

\section{Conclusões}

Mostramos nesta tese que as distribuições de Schwartz podem ser vistas como funções de classe $C^{\infty}$ definidas num certo espaço topológico e assumindo valores num corpo. Com isto foi contruído um novo ambiente algébrico e analítica em que equações diferenciais podem ser resolvidas fazendo uso de ferramentas clássicas de Cálculo. Estes novos ambientes foram chamados de Álgebas de Aragona. A seguir, foram usados para caracterizar a álgebra boolena e parametrizar o espectro maximal da álgebra plena de Colombeau.

Também estendemos resultados sobre a álgebra simplificada para a álgebra plena. Isto incluiu os conceitos de conjuntos e funções internos. Identificamos também o conjunto de zeros generalizadas de uma função holomorfa clássica dentro da teoria de conjuntos internos.

Estas contribuições devem ser úteis em continuar o projeto de fazer da Teoria das Funções Generalizadas de Colombeau uma teoria com suas ferramentas próprias e intrínsecas.

\subsection{Sugestões para Pesquisas Futuras}

Pretendemos continuar o estudo dos conjuntos de zeros de funções generalizadas e a sua ligação com fractais. Pretendemos também usar o ambiente das Álgebras de Aragona para estudar equações diferenciais e procurar por soluções que, usando métodos clássicos, não podem ser encontradas. Estas contribuições já estão em andamento com pesquisadores da área. Os resultados deste trabalho foram apresentados num congresso da área no mês de julho e um artigo está sendo submetido numa revestia internacional com arbitragem. 
54 CONCLUSÕES 


\section{Referências Bibliográficas}

[AB91a] Jorge Aragona e Hebe A Biagioni. Intrinsic definition of the colombeau algebra of generalized functions. Analysis Mathematica, 17(2):75-132, 1991. 4, 8

[AB91b] Jorge Aragona e Hebe A Biagioni. Intrinsic definition of the colombeau algebra of generalized functions. Analysis Mathematica, 17(2):75-132, 1991. 10

[AFJ05] J Aragona, Roseli Fernandez e SO Juriaans. A discontinuous colombeau differential calculus. Monatshefte für Mathematik, 144(1):13-29, 2005. 12, 15, 26, 27, 30, 31, 33

[AFJ' 09] Jorge Aragona, Roseli Fernandez, Stanley O Juriaans et al. Natural topologies on colombeau algebras. Topological methods in nonlinear analysis, 34(1):161-180, 2009. 11, 39, 40

[AFJ012] Jorge Aragona, Roseli Fernandez, Stanley O. Juriaans e Michael Oberguggenberger. Differential calculus and integration of generalized functions over membranes. Monatsh. Math., 166(1):1-18, 2012. 1, 37

[AGJ13] Jorge Aragona, ARG Garcia e SO Juriaans. Algebraic theory of colombeau $\hat{E}_{\frac{1}{4}}^{1} s$ generalized numbers. Journal of Algebra, 384:194-211, 2013. 25, 33

[AJ01] J Aragona e SO Juriaans. Some structural properties of the topological ring of colombeau's generalized numbers. 2001. 13, 25, 27, 28, 29, 32

[AJOS08a] J Aragona, SO Juriaans, Oswaldo Rio Branco de Oliveira e D Scarpalezos. Algebraic and geometric theory of the topological ring of colombeau generalized functions. Proceedings of the Edinburgh Mathematical Society, 51(3):545-564, 2008. 25, 26, 33, 35

[AJOS08b] J Aragona, SO Juriaans, Oswaldo Rio Branco de Oliveira e D Scarpalezos. Algebraic and geometric theory of the topological ring of colombeau generalized functions. Proceedings of the Edinburgh Mathematical Society, 51(3):545-564, 2008. 50

[BGR84] S. Bosch, U. Güntzer e R. Remmert. Non-Archimedean analysis, volume 261 of Grundlehren der Mathematischen Wissenschaften [Fundamental Principles of Mathematical Sciences]. Springer-Verlag, Berlin, 1984. A systematic approach to rigid analytic geometry. 19, 29

[BJ95] Tomek Bartoszynski e Haim Judah. Set Theory: on the structure of the real line. AK Peters/CRC Press, 1995. 17 
[Col82] JF Colombeau. New generalized functions multiplication of distributions physical applications contribution of $j$. sebastiao e silva. Quaderni di Matematica, 1982(11), 1982. 3

[Cor] A. R. G. Silva S. H. Cortes, W. Garcia. A discontinuous differential calculus in the framework colombeau's full algebra. preprint. 27

[Gar06] Antonio Ronaldo Gomes Garcia. Os números e as funções generalizadas plenas de Colombeau: aspectos algébricos, topológicos e analíticos. Tese de Doutorado, 2006. 2, 10

[JW95] Winfried Just e Martin Weese. Discovering modern set theory: Bd. 1 the basics. 1995. 18

[Khe] D. Khelif, A. Scarpalezos. Maximal closed ideals of colombeau algebras of generalized function. preprint. 20, 21

[KS06] A Khelif e Dimitris Scarpalezos. Zeros of generalized holomorphic functions. Monatshefte für Mathematik, 149(4):323-335, 2006. 20

[Kun96] Michael Kunzinger. Lie transformation groups in Colombeau algebras. na, 1996. 3, $5,6,7,9$

[Nig13] Eduard A Nigsch. Point value characterizations and related results in the full colombeau algebras article amssymb empty $g^{\wedge} e(\omega)$ and article amssymb empty $g^{\wedge} d(\omega)$. Mathematische Nachrichten, 286(10):1007-1021, 2013. 2, 10, 37

[OK99] M. Oberguggenberger e M. Kunzinger. Characterization of Colombeau generalized functions by their pointvalues. Math. Nachr., 203:147-157, 1999. 37

[OV08] M Oberguggenberger e Hans Vernaeve. Internal sets and internal functions in colombeau theory. Journal of Mathematical Analysis and Applications, 341(1):649-659, 2008. 2, 10, 37, 44, 47

[Tod06] T.D. Todorov. Pointwise values and fundamental theorem in the algebra of asymptotic functions. $\operatorname{arxiv} \cap$, 2006. 30

[TV08] Todor D Todorov e Hans Vernaeve. Full algebra of generalized functions and non-standard asymptotic analysis. Logic and Analysis, 1(3-4):205, 2008. 30

[Ver10] Hans Vernaeve. Ideals in the ring of colombeau generalized numbers. Communications in Algebraß, 38(6):2199-2228, 2010. 28, 32

[Ver11] Vernaeve. Nonstandard principles for generalized functions. J. Math. Anal. Appl., 384(2):536-548, 2011. 16, 17, 18, 19, 37, 48 